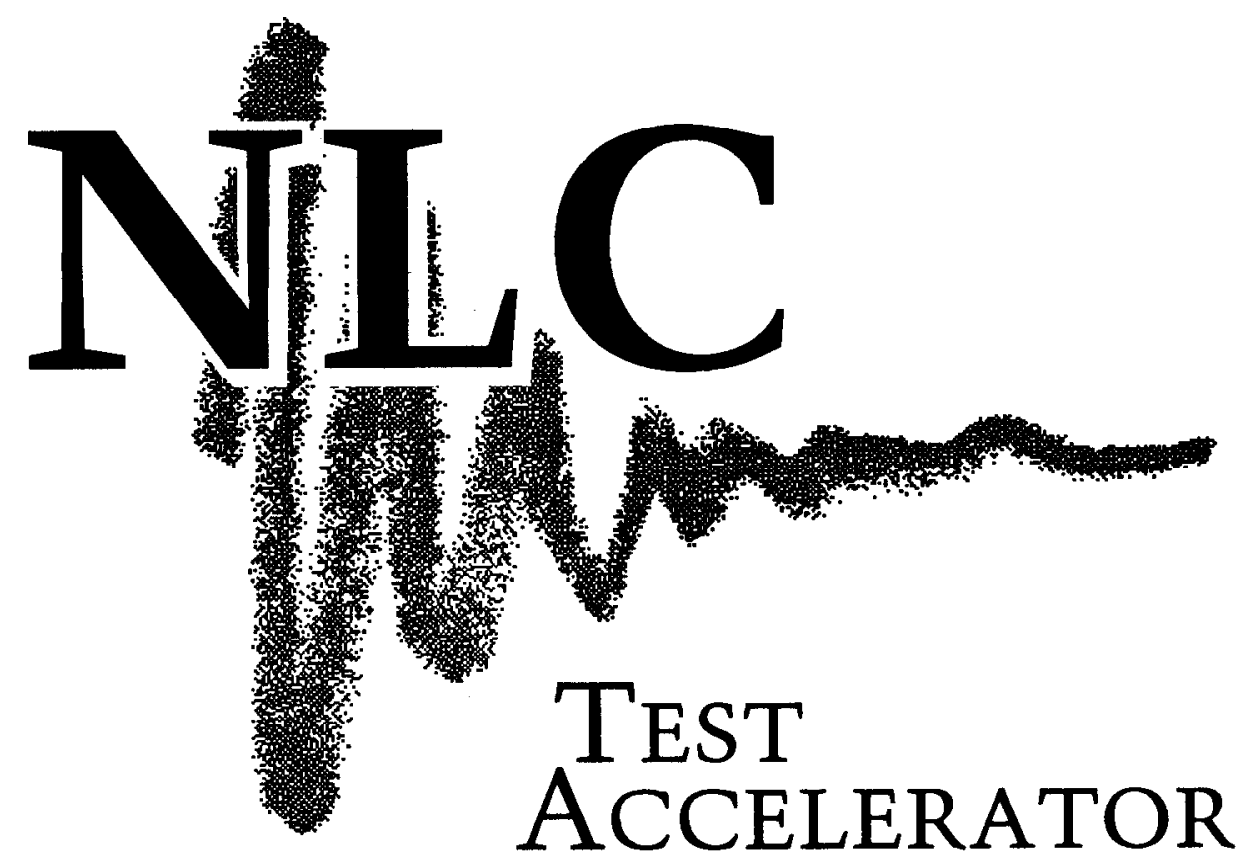

\title{
CONCEPTUAL DESIGN REPORT
}

SLAC-Report-411

August 1993

Prepared for the U. S. Department of Energy

under contract DE-AC03-76SF00515 
This document and the material and data contained therein, was developed under sponsorship of the United States Government. Neither the United States nor the Department of Energy, nor the Leland Stanford Junior University, nor their employees, nor their respective contractors, subcontractors, or their employees, makes any warranty, express or implied, or assumes any liability or responsibility for accuracy, completeness or usefulness of any information, apparatus, product or process disclosed, or represents that its use will not infringe privately-owned rights. Mention of any product, its manufacturer, or suppliers shall not, nor is it intended to, imply approval, disapproval, or fitness for any particular use. A royalty-free, nonexclusive right to use and disseminate same for any purpose whatsoever, is expressly reserved to the United States and the University. 


\title{
NEXT LINEAR COLLIDER TEST ACCELERATOR CONCEPTUAL DESIGN REPORT
}

\author{
SLAC-Report-411 \\ August 1993 \\ Stanford Linear Accelerator Center \\ Stanford University \\ Stanford, California 94309
}

Prepared for the U. S. Department of Energy under contract DE-AC03-76SF00515.

Printed in the United States of America. Available from the National Technical Information Service, U. S. Department of Commerce,'5285 Port Royal Road, Springfield, Virginia 22161. 


\section{Table of Contents}

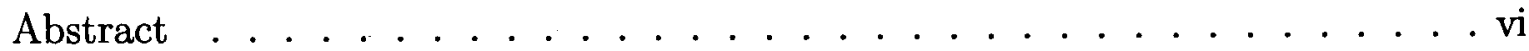

1. Introduction $\ldots \ldots \ldots \ldots \ldots \ldots$

1.1 Physics at the Next Linear Collider . . . . . . . . . . . . 1

1.2 Next Linear Collider Parameters . . . . . . . . . . . . . . 2

1.3 Test Accelerator Goals . . . . . . . . . . . . . . . . 5

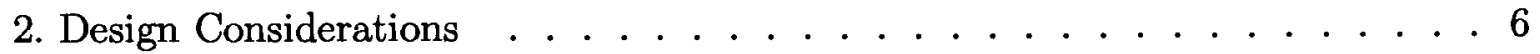

2.1 Overview . . . . . . . . . . . . . . . 6

2.2 RF System Overview and Performance . . . . . . . . . . 13

2.3 Klystron Development . . . . . . . . . . . . . . . 17

2.4 Accelerator Structure . . . . . . . . . . . . . . . . . . . 22

2.5 Transverse Wakefield Effects . . . . . . . . . . . . . . 28

2.6 Beam-Loading Compensation . . . . . . . . . . . . . . 32

2.7 Injector . . . . . . . . . . . . . . . . . 34

2.8 Chicane and Linac Beam Transport . . . . . . . . . . . . . 36

2.9 Spectrometer . . . . . . . . . . . . . . . . . . 39

2.10 Radiation Shielding $\ldots \ldots \ldots \ldots$. . . . . . . . 41

3. Accelerator Components . . . . . . . . . . . . . . . . 43

3.1 Overview . . . . . . . . . . . . . . . . 43

3.2 Conventional Facilities . . . . . . . . . . . . . . . . . . 43

3.3 Source . . . . . . . . . . . . . . . . 51

3.4 Vacuum System . . . . . . . . . . . . . . . . . . 52

3.5 Magnets . . . . . . . . . . . . . . . . . 53

3.6 Magnet Power Supplies . . . . . . . . . . . . . . . . . 57

3.7 Beam Devices . . . . . . . . . . . . . . . . . . . . 58

3.8 RF Drive System . . . . . . . . . . . . . . . . . . 67

$3.9 \mathrm{RF}$ Control and Measurement . . . . . . . . . . . . . . . . . . . . 69

3.10 Pulse Modulators . . . . . . . . . . . . . . . 72

3.11 Klystrons . . . . . . . . . . . . . . . . . . 75

3.12 RF Pulse Compression and Power Transmission . . . . . . . . 77

3.13 Accelerator Sections . . . . . . . . . . . . . . 81

3.14 Protection Systems . . . . . . . . . . . . . . 84

3.15 Control System Architecture . . . . . . . . . . . . 86

3.16 Support and Alignment $\ldots \ldots \ldots$. . . . . . . . 88

4. Experiments . . . . . . . . . . . . . . . . . . . 90

4.1 Overview . . . . . . . . . . . . . . . 90 
4.2 System Studies . . . . . . . . . . . . . . . . . . . . 90

4.3 Unloaded Energy Gain . . . . . . . . . . . . . . . . . 91

4.4 Linac Field-Emission Current . . . . . . . . . . . . . . 91

4.5 Multi-Bunch Beam-Loading Compensation . . . . . . . . . . 92

4.6 Transverse Components of the Accelerating Field . . . . . . . . . . 92

4.7 Transverse Multi-Bunch Beam Breakup . . . . . . . . . . . 93

4.8 Studies with an Upgraded Injector . . . . . . . . . . . . . 94

5. Environment, Safety, and Health _ . . . . . . . . . . 95

5.1 Overview . . . . . . . . . . . . . . . 95

5.2 Fire Safety . . . . . . . . . . . . . . . . 95

5.3 Radiation Safety . . . . . . . . . . . . . . 95

5.4 High-Power Microwave System Safety . . . . . . . . . . . 97

5.5 Electrical Safety . . . . . . . . . . . . . . . . 97

5.6 Construction . . . . . . . . . . . . . . . . . 9.98

5.7 Emergency Preparedness . . . . . . . . . . . . . . . . 98

5.8 Environmental Protection . . . . . . . . . . . . . . . . . . . . 99

5.9 Hazardous Material Issues . . . . . . . . . . . . . . . . . . . . 99

6. Administration . . . . . . . . . . . . . . . 100

6.1 Project Management . . . . . . . . . . . . 100

6.2 Manpower . . . . . . . . . . . . . . . . . . . . . 104

6.3 Cost Estimate, Work Breakdown, and Spending . . . . . . . 105

6.4 Schedule . . . . . . . . . . . . . . . . . 111

6.5 Quality Assurance . . . . . . . . . . . . . . 115

Acknowledgements . . . . . . . . . . . . 116

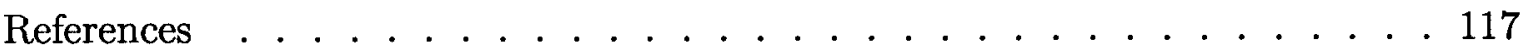




\section{Abstract}

This document presents the scientific justification and the conceptual design for the "Next Linear Collider Test Accelerator" (NLCTA) at SLAC. The goals of the NLCTA are to integrate the new technologies of X-band accelerator structures and rf systems being developed for the Next Linear Collider, to measure the growth of the "dark current" generated by rf field emission in the accelerator, to demonstrate multibunch beam-loading energy compensation and suppression of higher-order deflecting modes, and to measure any transverse components of the accelerating field.

The NLCTA will be a 42-meter-long beam line consisting, consecutively, of a thermionic-cathode gun, an X-band buncher, a magnetic chicane, six 1.8-meter-long sections of 11.4-GHz accelerator structure, and a magnetic spectrometer. Initially, the unloaded accelerating gradient will be $50 \mathrm{MV} / \mathrm{m}$. A higher-gradient upgrade option eventually would increase the unloaded gradient to $100 \mathrm{MV} / \mathrm{m}$. 


\section{Introduction}

\subsection{Physics at the Next Linear Collider}

Past and present colliding beam machines have provided spectacular views of the interactions of quarks and leptons at center-of-mass energies from a few $\mathrm{GeV}$ up to the masscs of the gauge bosons that mediate the electro-weak forces of nature. Experiments have confirmed that the Standard Model of these interactions provides an accurate description of particle physics in this energy domain. The confirmation of this model, however, is incomplete until we have determined the nature of the breaking of the exact $\mathrm{SU}(2)$ symmetry that is at its base. It is widely believed that to accomplish this goal, it will be necessary to explore particle physics at a centerof-mass energy much greater than the rest energies of the $W$ and $Z$ bosons. It is expected that the true source of the symmetry-breaking will be found in this regime, where the $W$ and $Z$ are relatively "light."

Development of the experimental physics program at $e^{+} e^{-}$colliders with centerof-mass energies at the $\mathrm{TeV}$ scale has progressed at workshops in the United States, ${ }^{1,2}$ Japan, and Europe. Studies of the top quark and its interactions, detailed examination of the interactions of gauge bosons, and searches for neutral scalars or other new particle states constitute the unique and essential program of scientific investigation to be carried-out at a collider with $0.5-\mathrm{TeV}$ center-of-mass energy. This is the first stage of the "Next Linear Collider" (NLC). Data samples of $3 \mathrm{fb}^{-1}$ (e.g., $3 \times 10^{32}$ $\mathrm{cm}^{-2} \mathrm{~s}^{-1}$ for $10^{7} \mathrm{~s}$ ) are sufficient to begin particle physics studies at this center-ofmass energy, while completion of the foreseeable experimental program at this stage may require the accumulation of $30 \mathrm{fb}^{-1}$ or more. The machine must reliably produce luminosities of $3 \times 10^{33} \mathrm{~cm}^{-2} \mathrm{~s}^{-1}$ to fulfill these goals. It is important for much of this physics program that the energy spread of the beam-beam collisions be kept small.

We consider it imperative that the design of the NLC be such that the beam energy can be increased over time to at least $1 \mathrm{TeV}$ in the center-of-mass. This energy provides access to new particle states at the $\mathrm{TeV}$ mass scale, and will open the $W-W$ scattering channel in which the source of electroweak symmetry-breaking must eventually take hold. The experimental program of a 1-TeV collider will require data samples of approximately $30 \mathrm{fb}^{-1}$ for initial success. Samples of several hundred $\mathrm{fb}^{-1}$ may be required to fully explore the physics. To accumulate these large data samples will require the NLC to reach luminosities of $10^{34} \mathrm{~cm}^{-2} \mathrm{~s}^{-1}$ or more at its highest energies. 


\subsection{Next Linear Collider Parameters}

To meet the above physics requirements, the Next Linear Collider (NLC) should have an initial center-of-mass energy of $0.5 \mathrm{TeV}$ (five times the SLC) with the capability of being upgraded to $1 \mathrm{TeV}$. This increase in energy can be obtained either by grcatly increasing the length of the collider (by a factor of 10 to 20) relative to the SLC, or by increasing both the accelerating field and the length to obtain the desired energy. The present consensus at SLAC ${ }^{5,6}$ is that we should first increase the accelerating field by about a factor of three to six-to about 50 to $100 \mathrm{MV} / \mathrm{m}$. To limit the rf power required, this field should be provided by structures similar to those used in the SLC, but at the higher of frequency of $11.4 \mathrm{GHz}$.

The choice of luminosity range also greatly influences the design of the NLC. In principle, one could increase the luminosity simply by raising the repetition rate of the accelerator, but the wall-plug power would increase in direct proportion. In a reasonable design, the wall-plug power should not exceed about $250 \mathrm{MW}$. Given this constraint, the best way to increase the luminosity is to shrink the beam size at the collision point. In addition, the beam cross section must be kept flat at the collision point in order to minimize the amount of "beamstrahlung" radiation emitted as energetic electrons or positrons interact with the electromagnetic field of the opposing bunch.

The luminosity can be further enhanced by accelerating several bunches within each machine pulse. A single bunch of particles can, in practice, extract only a few percent of the energy available in the accelerating structure. With additional bunches, both a greater luminosity and a higher efficiency of energy transfer to the beam are achieved. The number of particles in each bunch, another factor that directly affects the luminosity, is limited by the single-bunch beam loading in the accelerating structures, and by the amount of beamstrahlung radiation that can be tolerated at the final focus. The obvious solution is to generate a train of bunches, each with a fairly moderate number of particles, on each rf pulse.

Given these goals and constraints, we can sketch a rough design of a linear collider able to achieve both the desired energy and luminosity. ${ }^{5,6}$ A possible layout is shown in Figure 1. There are two complete linear accelerators, one for electrons and the other for positrons. Each linac is supplied with its electron or positron beam by a damping ring followed by a preacceleration system consisting of two bunch compressors and a $16-\mathrm{GeV}$ linac. After passing through the main linacs and final focus systems, the beams collide at a small crossing angle inside a particle detector.

To illustrate the basic features of the NLC operation, consider the transport of electrons through the collider on a single machine pulse. A train of up to 100 bunches is created at the source and accelerated to about $1.8 \mathrm{GeV}$ in a preaccelerator. This bunch train then is injected into a damping ring that serves to reduce the transverse and longitudinal phase space occupied by the electrons in each bunch. At the proper moment, these bunches are extracted from the ring and compressed along 


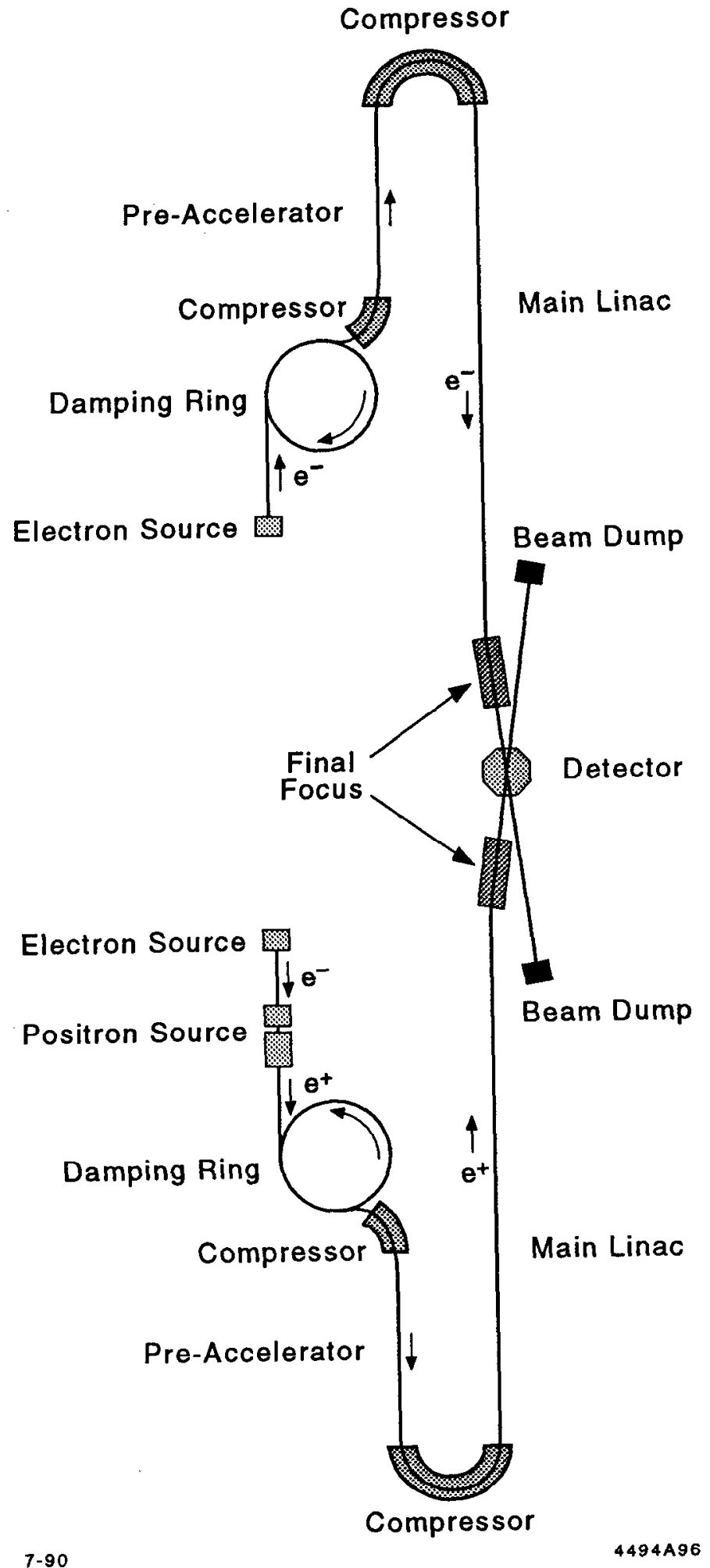

Figure 1. Possible layout of the Next Linear Collider. 
their direction of motion by a bunch compressor, after which they are accelerated to about $16 \mathrm{GeV}$ and compressed a second time just prior to injection into the main (high-gradient) linac. The entire bunch train is carefully steered and focused as the electrons are accelerated to full energy in the linac. Precision magnets in the final focus system squeeze the bunches transversely by about a factor of 300 just before they collide with similar bunches of positrons. Except for the fact that the positrons are created from the interactions that occur when a bunch of electrons hits a metal target, their transport is similar to that of the electrons. After the beams collide, their debris is channeled out of the detector area and into shielded dumps.

The parameters of the NLC have not been finalized. However, the range of possibilities has been narrowed considerably over the past few years. ${ }^{5-9}$ At SLAC, we have developed sets of parameters for an NLC which has an initial center-of-mass energy of $0.5 \mathrm{TeV}$, and an upgraded center-of-mass energy of $1 \mathrm{TeV}$. These parameters are listed in Table 1. The upgrade is performed by adding more rf power to the linacs, and by modifying the final focus regions.

Table 1. NLC Parameters

\begin{tabular}{|l|c|c|}
\hline & Initial & Upgraded \\
\hline Energy per beam & $250 \mathrm{GeV}$ & $500 \mathrm{GeV}$ \\
Luminosity & $8 \times 10^{33}$ & $2 \times 10^{34}$ \\
Linac length (both linacs) & $15 \mathrm{~km}$ & $15 \mathrm{~km}$ \\
Unloaded accelerating gradient & $50 \mathrm{MV} / \mathrm{m}$ & $100 \mathrm{MV} / \mathrm{m}$ \\
RF frequency & $11.4 \mathrm{GHz}$ & $11.4 \mathrm{GHz}$ \\
Electrons per bunch & $0.65 \times 10^{10}$ & $1.3 \times 10^{10}$ \\
Bunches & 90 & 65 \\
Repetition rate & $180 \mathrm{~Hz}$ & $120 \mathrm{~Hz}$ \\
Wall-plug power & $160 \mathrm{MW}$ & $250 \mathrm{MW}$ \\
Bunch height at collision & $3 \mathrm{~nm}$ & $2 \mathrm{~nm}$ \\
Bunch width at collision & $300 \mathrm{~nm}$ & $430 \mathrm{~nm}$ \\
Bunch length at collision & $100 \mu \mathrm{m}$ & $100 \mu \mathrm{m}$ \\
\hline
\end{tabular}




\subsection{Test Accelerator Goals}

The NLC parameter sets discussed in the previous section were based on rf linacs with unloaded accelerating gradients of $50 \mathrm{MeV} / \mathrm{m}$, upgradable to $100 \mathrm{MeV} / \mathrm{m}$, at the $\mathrm{rf}$ frequency of $11.4 \mathrm{GHz}$. During the past several years much experience has been gained with this rf frequency at SLAC and KEK. We have powered 11.4-GHz structures to reach peak surface fields in excess of $500 \mathrm{MV} / \mathrm{m}$, and have achieved an accelerating gradient of $100 \mathrm{MV} / \mathrm{m}$ stably, for an extended period of time, in a 30 -cell structure. High-power klystrons have been constructed which produce 50MW pulses of 1- $\mu$ s duration. We have constructed high-power rf pulse-compression systems which achieve a factor of five in peak-power multiplication. Designs for more efficient modulators have recently been completed. Finally, we are developing lowloss microwave components for manipulating and transmitting $11.4-\mathrm{GHz}$ high-power pulses.

Many of the outstanding questions related to achieving the NLC luminosity can be answered using the SLAC Final Focus Test Beam, and with dedicated experiments at the SLC. However, the details of the trade-off between linac length and accelerating gradient cannot be evaluated until a test has been made of a complete $11.4-\mathrm{GHz}$ accelerating system. The primary missing ingredient for completing the NLC rf-system design is a test accelerator based on $11.4-\mathrm{GHz}$ technology and designed specifically to study the range of parameters of interest for the NLC. ${ }^{10,11}$

One goal of the Next Linear Collider Test Accelerator (NLCTA) is to construct and operate reliably a high-gradient X-band linac in order to integrate the new technologies of X-band accelerator structures and rf systems being developed for the NLC. The NLCTA will serve as a test bed as the design of the NLC evolves, and will provide a model upon which a reliable cost estimate for the rf system of an NLC linac can be based.

Other goals are to measure the growth of the accelerated "dark current" generated by rf field emission in full-length accelerator sections, to demonstrate multi-bunch beam-loading energy compensation at the $0.1 \%$ level for $25 \%$ steady-state loading, to observe the suppression of the higher-order deflecting modes of the accelerator structure, and to measure any transverse components of the accelerating field. As part of this overall program, we will study the dynamics of the beam during the high-gradient acceleration of many bunches on each rf pulse of the X-band linac. 


\section{Design Considerations}

\subsection{OVERVIEW}

The Next Linear Collider Test Accelerator (NLCTA) will be a 42-meter-long beam line consisting, consecutively, of an injector, a chicane, a linac, and a spectrometer.

The injector will consist of a 150-kV gridded thermionic-cathode gun, an X-band prebuncher, a capture section, and a preacceleration section. Downstream from the injector will be a magnetic chicane for longitudinal phase-space manipulation. After energy collimation, the average current injected into the linac will be comparable to the NLC specification, $0.65 \times 10^{10} e /(1.4 \mathrm{~ns})$.

The NLCTA linac will consist of six 1.8-meter-long X-band accelerator sections which are detuned for higher-order-mode wakefield suppression. These sections will be powered by three 50-MW klystrons whose peak power will be quadrupled by SLED-II rf pulse compressors. This will yield an unloaded acceleration gradient of $50 \mathrm{MV} / \mathrm{m}$ so that the maximum energy gain of the beam in the X-band linac will be $540 \mathrm{MeV}$. The NLCTA rf system parameters are listed in Table 2.

Downstream from the linac will be a magnetic spectrometer that will analyze the bunch train after acceleration. A vertical kicker magnet in the spectrometer will provide a method for separating the bunches vertically so that the energy and energy spread may be measured along the bunch train. It will also be possible to measure emittance in the spectrometer and in the chicane.

We plan in the future to increase the linac gradient to $100 \mathrm{MV} / \mathrm{m}$ by replacing the three 50-MW klystrons which initially will power the linac with six 100-MW klystrons, as indicated in Table 2.

We plan also in the future to upgrade the injector in order to increase the bunch spacing and intensity, cach by a factor of 16 . This will permit more detailed beam-dynamics studies on a train of bunches similar to that required for the NLC.

Views of the NLCTA are shown in Figures 2 through 6 . We plan to locate the NLCTA in End Station B (ESB) in a configuration that allows access to the overhead crane from the sliding concrete door on the south wall, and that retains the option to use existing Beam Lines 19 and 20 for future test beams if desired. The NLCTA will be contained in a six-foot-thick concrete shielding enclosure to be constructed inside ESB. The modulators, klystrons, and power supplies will be located next to this enclosure, inside ESB, in order to allow access to them while the NLCTA is running. A control room will be located outside, adjacent to the south wall of ESB. 
Table 2. NLCTA RF System Parameters

\begin{tabular}{|l|c|c|}
\hline \multicolumn{1}{|c|}{ Parameter } & $\begin{array}{c}\text { NLCTA } \\
\text { Design }\end{array}$ & $\begin{array}{c}\text { Energy } \\
\text { Upgrade }\end{array}$ \\
\hline Linac unloaded energy gain & $540 \mathrm{MeV}$ & $1080 \mathrm{MeV}$ \\
Linac active length & $10.8 \mathrm{~m}$ & $10.8 \mathrm{~m}$ \\
Unloaded accelerating gradient & $50 \mathrm{MV} / \mathrm{m}$ & $100 \mathrm{MV} / \mathrm{m}$ \\
Injection energy & $90 \mathrm{MeV}$ & $90 \mathrm{MeV}$ \\
RF frequency & $11.424 \mathrm{GHz}$ & $11.424 \mathrm{GHz}$ \\
Number of klystrons & 3 & 6 \\
Klystron peak power & $50 \mathrm{MW}$ & $100 \mathrm{MW}$ \\
Klystron pulse length & $1.5 \mu \mathrm{s}$ & $1.5 \mu \mathrm{s}$ \\
RF pulse compression power gain & 4.0 & 4.0 \\
Phase advance per cell & $2 \pi / 3$ & $2 \pi / 3$ \\
HOM suppression technique & Detuning & Detuning \\
\hline
\end{tabular}




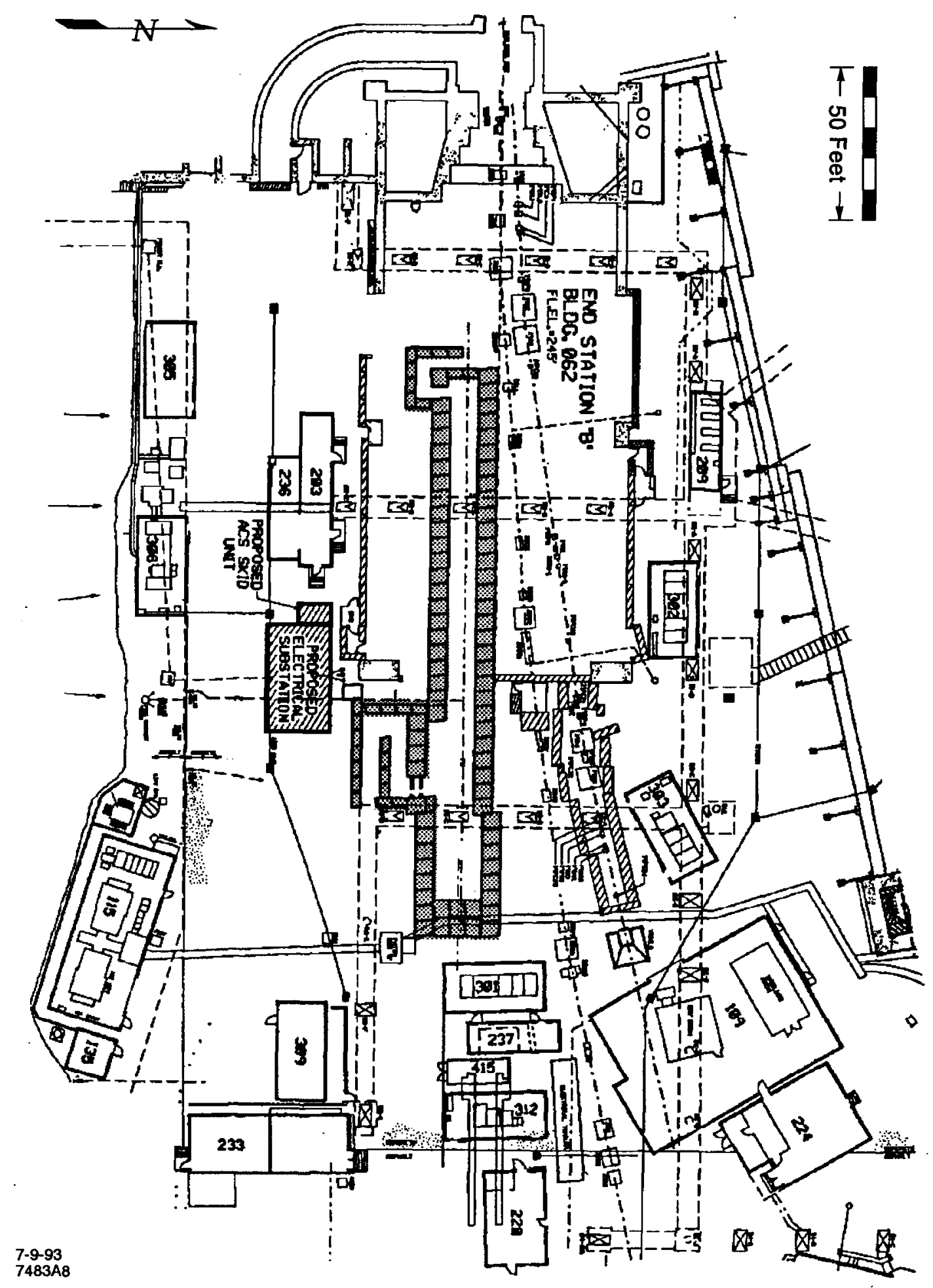

Figure 2. Plan view of the NLCTA site showing the area inside and around End Station B at SLAC. The shielding enclosure for the NLCTA is shaded. 


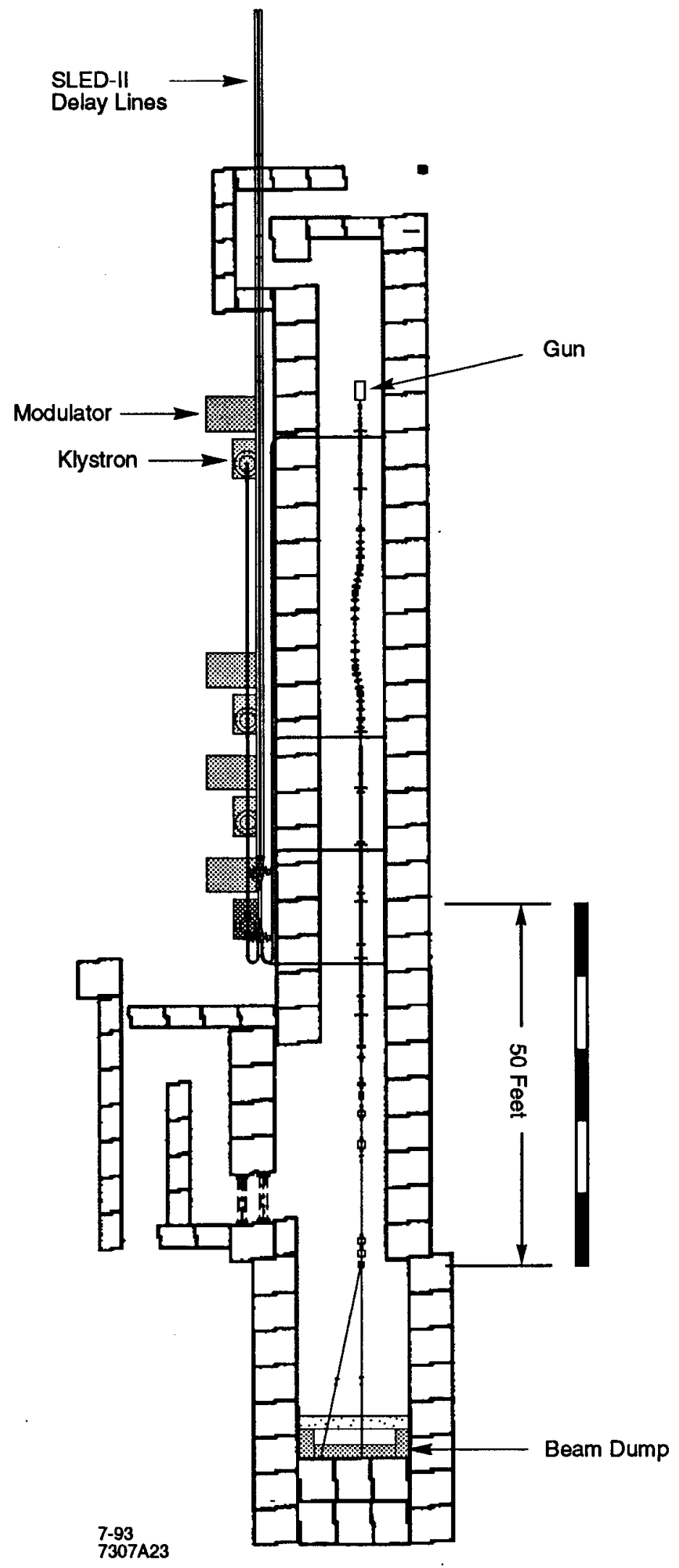

Figure 3. Plan view of the NLCTA in its shielding enclosure. 

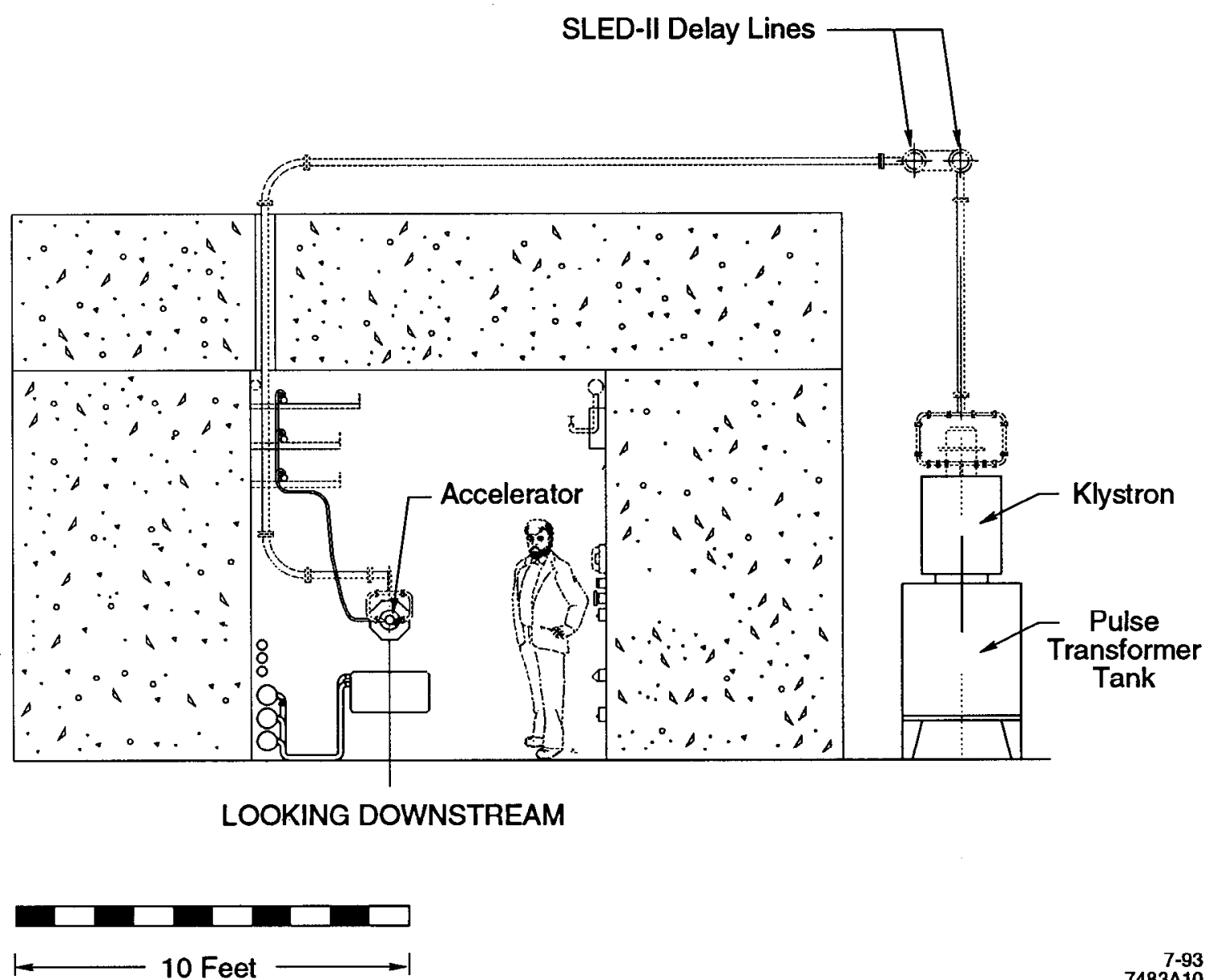

Figure 4. Cross-sectional view of the NLCTA linac. 


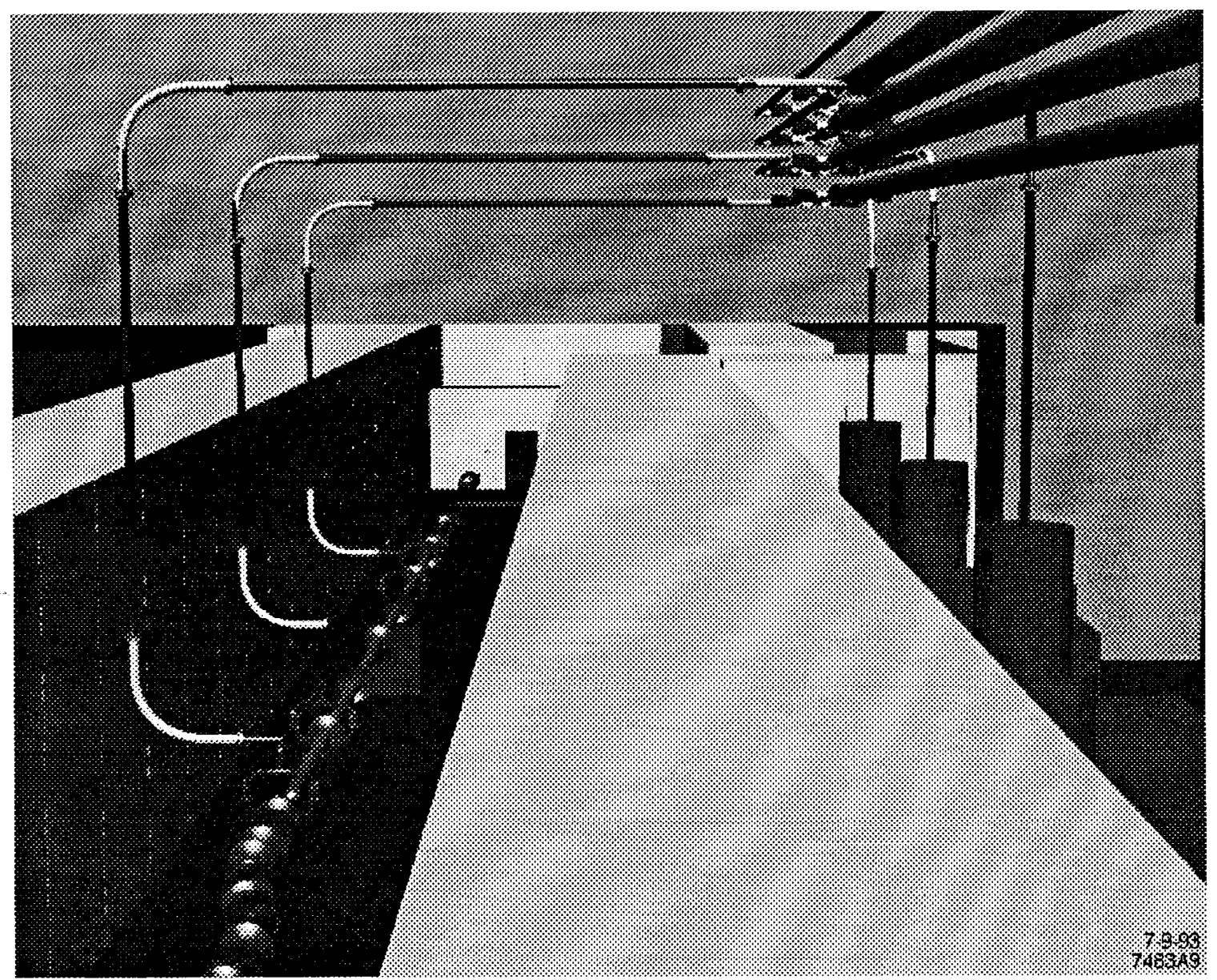

Figure 5. View looking down the NLCTA linac with the roof shielding removed. The beam line is visible to the left of the shielding wall. The klystrons and modulators are visible to the right. 


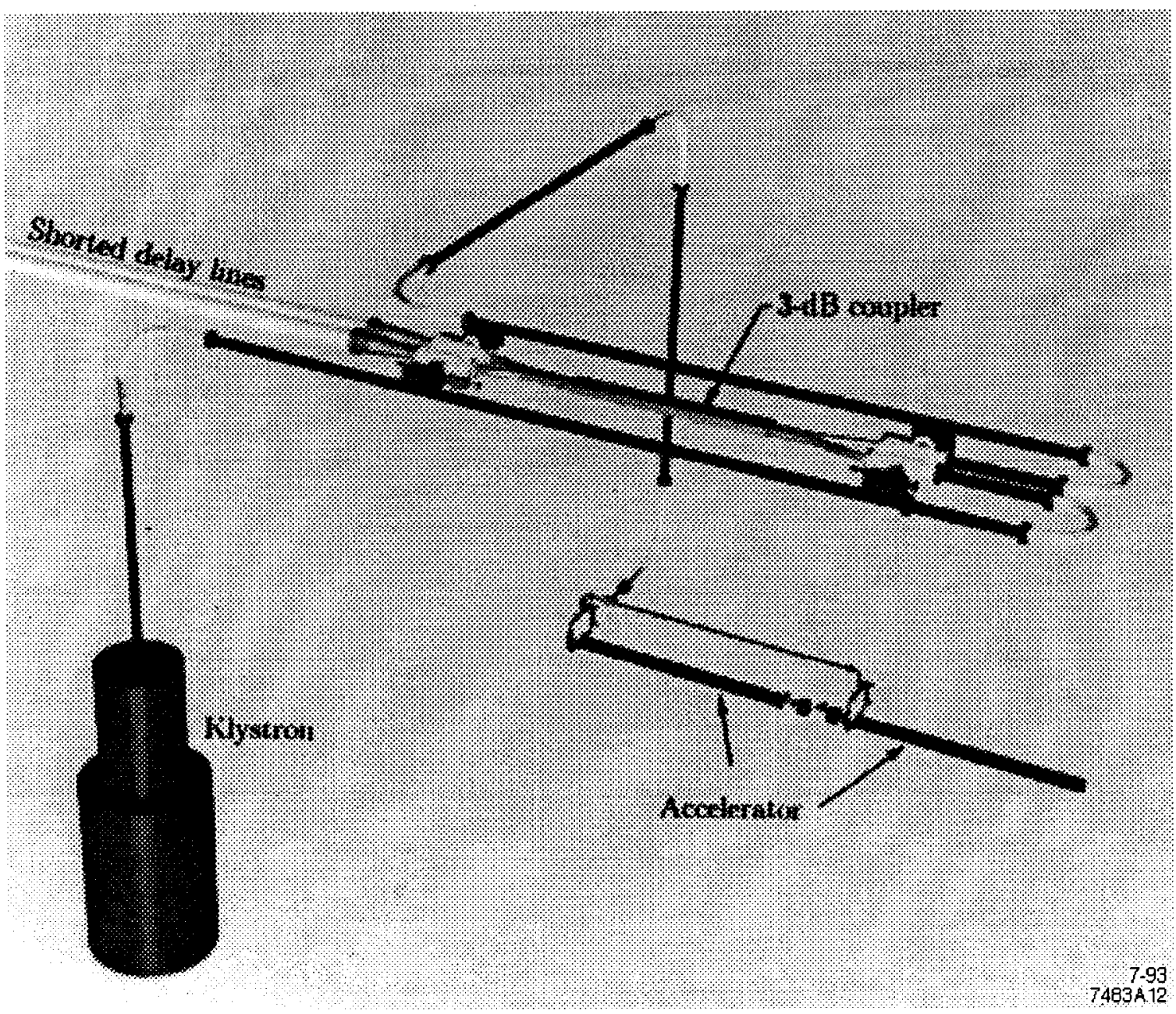

Figure 6. Layout of one NLCTA rf station consisting of klystron, SLED-II pulse compressor, and two accelerator sections. (The accelerator shielding is not shown.) 


\subsection{RF System OVERVIEW AND PERFormance}

\subsubsection{Overview}

Peak power of $200 \mathrm{MW}$ will be required to drive a pair of $1.8-\mathrm{m}$-long sections of the NLCTA accelerator structure to an accelerating gradient of $50 \mathrm{MV} / \mathrm{m}$. An rf pulse length of $250 \mathrm{~ns}$ at the accelerator input will provide $100 \mathrm{~ns}$ for filling the structure, $125 \mathrm{~ns}$ for accelerating the bunch train, and $25 \mathrm{~ns}$ for rise- and fall-times.

The rf sources for the accelerator will be four 50-MW klystrons positioned along the accelerator, outside the shielded enclosure. One of the klystrons will power the prebuncher and preacceleration sections of the injector. The other three klystrons will power the six-section linac. Each klystron will be pulsed by an independent modulator. This will allow flexibility for multi-bunch energy control. It also will allow adequate modulator power for a future energy upgrade in which the three 50-MW linac klystrons are replaced by six 100-MW klystrons in order to achieve a $100-\mathrm{MV} / \mathrm{m}$ linac gradient, as indicated in Table 2.

Each klystron will feed a SLED-II rf pulse compressor, ${ }^{12-15}$ which will compress the $50-\mathrm{MW}, 1.5-\mu \mathrm{s}$-long klystron pulse by a factor of six in time to $0.25 \mu \mathrm{s}$ and, in doing so, will multiply the peak power by a factor of four, to $200 \mathrm{MW}$. Each SLED-II pulse compressor will have a pair of 36-meter-long low-loss waveguide delay lines. The delay lines of all four pulse compressors will extend parallel to the accelerator, overlapped with one another, outside the shielded accelerator vault.

The output of each SLED-II pulse compressor will be transmitted in low-loss oversized circular waveguide that will enter the shielded vault through a penetration in a roof block. Inside the shielded vault, a mode transducer will split the power from the oversized circular guide into two short rectangular waveguides, each of which will feed one accelerator section. The layout of the accelerator, klystrons, SLED-II pulse compressors, and waveguides is shown in Figures 3 through 6.

In the future, we plan to upgrade the NLCTA rf system to double the accelerating gradient to $100 \mathrm{MV} / \mathrm{m}$. This would require klystrons (or other rf sources) producing about $200 \mathrm{MW}$ (or pairs of sources, each producing $100 \mathrm{MW}$ ). A two-stage rf pulse-compression system is another possibility, which would reduce the power source requirement to about $120 \mathrm{MW}$ per source (or $60 \mathrm{MW}$ per source, if deployed in pairs).

In Table 3, the peak power and energy per pulse are tracked backward through the rf system, starting at the accelerator feeds, for accelerating gradients of $50 \mathrm{MV} / \mathrm{m}$ (the "NLCTA Design") and $100 \mathrm{MV} / \mathrm{m}$ (the "Energy Upgrade"). The following sections expand on the RF System performance goals presented in Table 3.

\subsubsection{RF Power Transmission}

Power will be transmitted from the output of the SLED-II rf pulse-compressor to the feeds of the accelerating structures with an efficiency of about $88 \%$. This estimate 
Table 3. NLCTA RF System Performance

\begin{tabular}{|l|c|c|}
\hline & $\begin{array}{c}\text { NLCTA } \\
\text { Design }\end{array}$ & $\begin{array}{c}\text { Energy } \\
\text { Upgrade }\end{array}$ \\
\hline Accelerator Structure & & \\
Gradient (MV/m) & 50 & 100 \\
Pulse length (ns) & 250 & 250 \\
Peak power per feed (MW) & 87 & 350 \\
Pulse energy per feed (J) & 22 & 87 \\
Feeds per klystron & 2 & 1 \\
Power Transmission Efficiency & 0.86 & 0.86 \\
Pulse Compression & & \\
Peak output power (MW) & 200 & 800 \\
Pulse energy (J) & 50 & 200 \\
Power gain & 4.0 & 4.0 \\
Compression ratio & 6 & 6 \\
Compression efficiency & 0.67 & 0.67 \\
Klystron & & \\
Peak output power (MW) & 50 & 100 \\
RF pulse length ( $\mu$ s) & 1.5 & 1.5 \\
Klystron efficiency & 0.45 & 0.45 \\
Modulator & & \\
Klystrons per modulator & 1 & 2 \\
Peak output power (MW) & 110 & 440 \\
Modulator efficiency & 0.73 & 0.73 \\
PFN stored energy (J) & 220 & 870 \\
AC input power (kW) at 180 pps & 41 & 165 \\
RF System Net Efficiency & 0.19 & 0.19 \\
\hline
\end{tabular}


is based on theoretical calculations and measurements of prototypes. The losses of the individual components of the power-transmission system will be: $1 \%$ for 10 meters of 3 -inch-diameter circular waveguide, $2 \%$ each for three $90^{\circ}$ circular waveguide bends, $2 \%$ for one power-splitting mode transducer, and $3 \%$ for the rectangular waveguide feeds to the accelerator. There will be an additional $2 \%$ loss in transmitting power from the klystron output to the input of the SLED-II pulse compressor (assuming one $90^{\circ}$ bend), giving a net power transmission efficiency of about $86 \%$ from klystron to accelerator.

\subsubsection{RF Pulse Compression}

The SLED-II rf pulse compressors ${ }^{12-15}$ will compress the $50-\mathrm{MW}, 1.5-\mu$ s-long klystron pulses by a factor of six in time to $0.25 \mu$ s and, in doing so, will multiply the peak power by a factor of four, to $200 \mathrm{MW}$, with a compression efficiency of $67 \%$. As shown in Figure 6, the main components of the SLED-II rf pulse compressor are a 3-dB coupler (power divider) and two shorted, 36-m-long, 4.75-inch-diameter, circular-waveguide delay lines. The down-and-back transit time of each delay line is equal to the required compressed pulse length of $250 \mathrm{~ns}$. The delay lines are oversized, and operate in the $\mathrm{TE}_{01}$ mode, in order to reduce power losses.

SLED-II pulse compressors have an "intrinsic" inefficiency, as well as an inefficiency due to component losses. The intrinsic inefficiency is the result of energy incident on the delay lines being reflected from the delay line entrance aperture (an iris) prior to the formation of the high-peak-power SLED-II output pulse. Also, a small amount of rf energy remains in the delay lines after the output pulse. For pulse-length compression by a factor of six, the intrinsic efficiency of SLED-II is $75 \%$. (This is obtained with an iris-reflection coefficient of 0.685.) In addition, there is a $4 \%$ power loss in the 4.75-inch-diameter copper delay lines, and a $6 \%$ loss in the 3-dB coupler. This brings the net compression efficiency down to $67 \%$, as shown in Table 3 .

\subsubsection{Klystron Efficiency}

The NLCTA will be powered by four 11.424-GHz klystrons operating at a microperveance of $1.2(350 \mathrm{~A}$ at $440 \mathrm{kV}$ ). The goal of 50-MW peak klystron power requires only $33 \%$ beam-to-rf conversion efficiency. However, it is reasonable to expect that the klystron efficiency will be closer to $45 \%$, in which case the output power will exceed the $50-\mathrm{MW}$ goal by a comfortable $20-\mathrm{MW}$ margin. Our X-band klystron development work, and our plan for the NLCTA tubes, are discussed in Sections 2.3 and 3.11 , respectively.

\subsubsection{Modulator Performance}

An important consideration in modulator performance is the efficiency for the

* The pulse length of the SLED-II input pulse (klystron output pulse) can be any multiple of 250 ns. A larger compression factor gives a higher power gain, at the expense of reduced compression efficiency. 
transfer (through a pulse transformer) of energy stored in the capacitors of the pulseforming network (PFN) to useful energy in the flat-top portion of the output pulse. This efficiency is given roughly by $T_{p} /\left(T_{p}+\alpha T_{r}\right)$, where $T_{p}$ is the time duration of the flat-top, $T_{r}$ is the pulse rise-time, and $\alpha$ is a coefficient of order one. In turn, the risetime is roughly proportional to the turns-ratio of the pulse transformer. In the present modulator design (see Section 3.10), the transformer turns ratio is reduced by using a three-stage "multiplying Blumlein" design for the PFN. This allows a factor-of-three reduction in the transformer turns-ratio from about 20:1 to $6: 1$, a corresponding decrease in rise-time, and an increase in energy-transfer efficiency to about $77 \%$. The overall modulator efficiency quoted in Table 3 is $73 \%$, because of the fact that there is an additional $5 \%$ loss in the front-end modulator components (such as the dc power supply, transformers, charging choke, etc.). Finally, it should be noted that, although the use of an rf pulse-compression system introduces additional (intrinsic) losses, they are nearly compensated by the increase in modulator efficiency gained because of the longer klystron pulse length. Thus, the net efficiency of the rf pulse compressor and the modulator tends to remain at about $50 \%$, nearly independent of pulse length. Our plan for the NLCTA modulators is discussed in Section 3.10.

\subsubsection{Implications for the NLC}

The rf system performance in Table 3 can be extrapolated to a full-scale NLC. Assume $25 \%$ beam-loading so that the loaded gradient is $37.5 \mathrm{MV} / \mathrm{m}$. Then, the active structure length required for acceleration to $500 \mathrm{GeV}$ is $14 \mathrm{~km}$, assuming an additional $5 \%$ overhead due to off-crest operation, etc. The number of NLCTA units (3.6 meters of structure plus a power source) required would be 3890 . At $180 \mathrm{pps}$, the total wall-plug power for the rf system would be about $160 \mathrm{MW}$. In this scenario, one modulator could easily feed two klystrons, so only 1945 modulators would be required. However, the energy stored in the pulse-forming network would double to $440 \mathrm{~J}$, and the wall-plug power per modulator would double to $82 \mathrm{~kW}$.

An increase in the rf system efficiency would be very desirable. At a more favorable compression ratio of 5:1, and with some reduction in component losses due to continuing research and development, the efficiency of the rf pulse-compression system could be increased to about $77 \%$. Assuming that the modulator efficiency were improved slightly (from $73 \%$ ) to $75 \%$, that the power transmission efficiency were increased (from $86 \%$ ) to $92 \%$, and that the klystron efficiency were increased (from $45 \%$ ) to $50 \%$, then the overall rf system efficiency would be increased (from $19 \%$ ) to $27 \%$ and the wall-plug power for the above example would be reduced (from $160 \mathrm{MW}$ ) to $115 \mathrm{MW}$.

If the energy of the NLC were doubled to $500 \mathrm{GeV}$ per beam by increasing the gradient to $100 \mathrm{MV} / \mathrm{m}$, if the repetition rate were reduced to $120 \mathrm{pps}$, and if the number of bunches were reduced to 65 (reducing the rf pulse length at the accelerator to $200 \mathrm{~ns}$ ), then the wall-plug power would be about $250 \mathrm{MW}$, again assuming that the above higher efficiencies had been achieved. 


\subsection{Klystron DeVelopment}

A high-power X-band klystron development project has been underway at SLAC since $1988^{16-18}$ The project was started with the goal of developing a 100-MW tube for service in a future NLC. Since then, eight experimental tubes in the "XC" series, all variations on this theme, were designed and tested. Experience gained from the $\mathrm{XC}$ klystron project is now being used to develop 50-MW ("XL") klystrons with 1.5$\mu$ s pulse-length for the NLCTA. The 100-MW development work will not be resumed until after the design, production, and testing of the 50-MW klystrons for the NLCTA are completed. The basic parameters of the X-band klystrons in the XC and XL series are shown in Table 4.

Table 4. X-Band Klystron Design Parameters

\begin{tabular}{|l|c|c|c|}
\hline Parameter & $\begin{array}{r}\text { XC-Series } \\
\text { Klystrons }\end{array}$ & $\begin{array}{c}\text { XL-Series } \\
\text { Klystrons }\end{array}$ & $\begin{array}{c}\text { NLC } \\
\text { Klystrons }\end{array}$ \\
\hline Operating frequency $(\mathrm{GHz})$ & 11.424 & 11.424 & 11.424 \\
Peak output power $(\mathrm{MW})$ & 100 & 50 & 100 \\
Rf pulse width $(\mu \mathrm{s})$ & 1 & 1.5 & 1.5 \\
Pulse repetition rate (pps) & 180 & 180 & 180 \\
Rf pulse rise-time (ns) & $\leq 10$ & $\leq 10$ & $\leq 10$ \\
Microperveance $\left(\mu A / V^{3 / 2}\right)$ & 1.9 & 1.2 & 1.2 \\
Beam voltage $(\mathrm{kV})$ & 440 & 440 & 550 \\
Beam current $(\mathrm{A})$ & 550 & 350 & 490 \\
Beam-to-rf efficiency & 0.40 & 0.45 & 0.45 \\
Saturation gain $(\mathrm{dB})$ & 55 & 55 & 55 \\
Focusing field $(\mathrm{kG})$ & 5.7 & 5.0 & 5.3 \\
\hline
\end{tabular}

\subsubsection{Summary of the XC-Series Klystron Experiments}

The microperveance desired for a long-lived 100-MW NLC tube is 1.2 (490 A at $550 \mathrm{kV}$ ). However, limited by the capability of the existing pulse modulators, the $\mathrm{XC}$ prototypes were designed and tested at the higher microperveance of $1.9(550 \mathrm{~A}$ at $440 \mathrm{kV}$ ). Consequently, cathode life-times of the experimental XC tubes were expected to be short relative to production tubes of lower perveance because of the high cathode loading (up to $25 \mathrm{~A} / \mathrm{cm}^{2}$ ). The power demonstrated in the tests of five representative tubes of the eight in the XC-series is shown in Figure 7. 


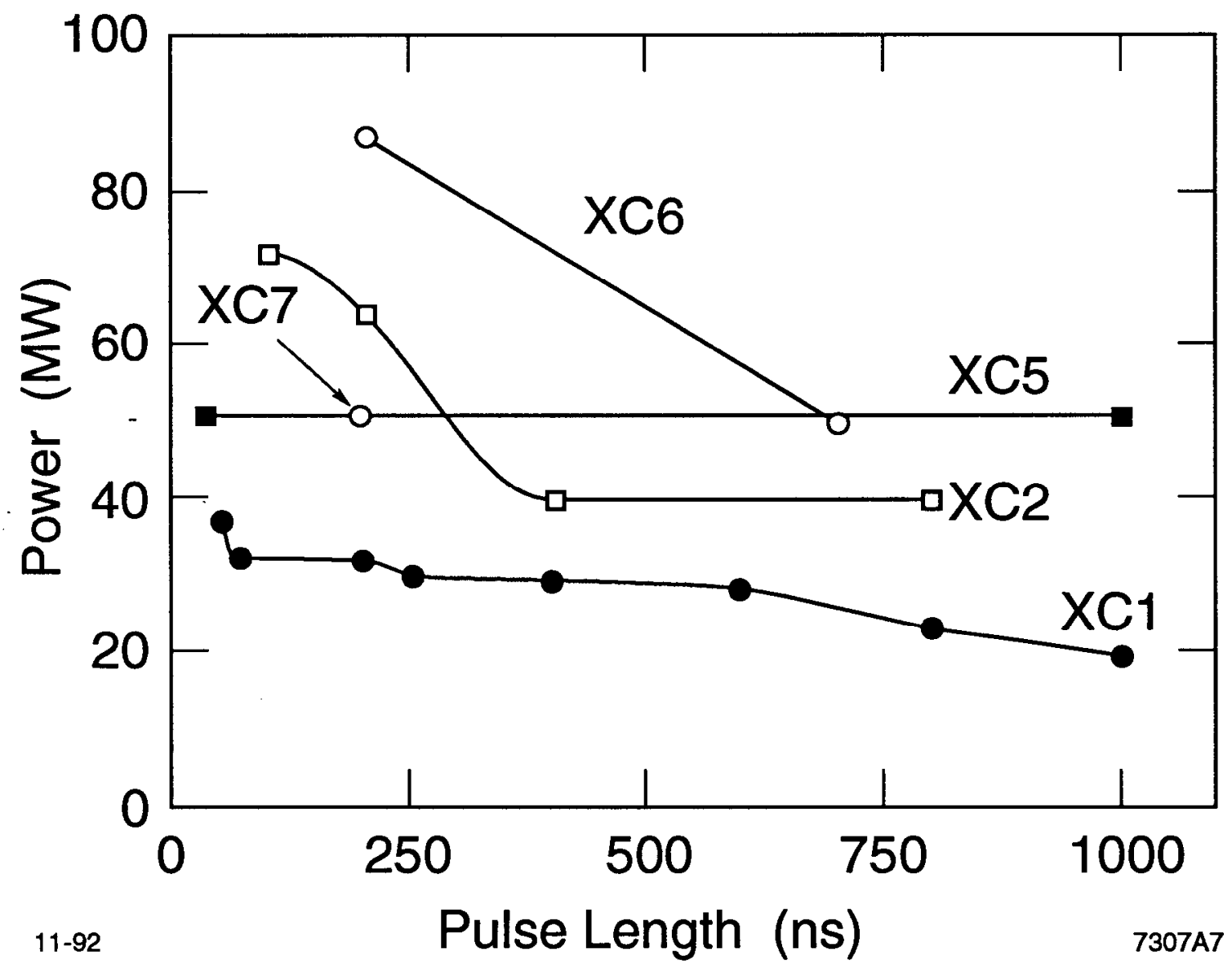

Figure 7. Power capability of the experimental XC klystrons. 
The first five tubes had in common the designs of their input, gain, and pcnultimate cavities. The output cavities of $\mathrm{XC} 1$ through $\mathrm{XC} 4$ included various arrangements of single-gap, and inductively coupled double-gap output cavities of the same beam-tunnel diameter as the input cavities. These first four tubes showed promise in terms of peak-power production at short pulse lengths, with a maximum of $72 \mathrm{MW}$. However, none was capable of $50 \mathrm{MW}$ at pulse lengths of $1-\mu \mathrm{s}$ or longer. Later inspection of XC1-XC4 showed severe damage to the output cavities.

The fourth klystron (XC4) included a new electron gun design which improved beam transmission. Failure to obtain an improvement in either performance or survivability ruled out beam quality as the primary cause of failure at long pulse lengths.

Based on the evidence, two modifications were made to the output circuit: (1) The beam-tunnel diameter was increased to better isolate the microwave circuit from the beam. (2) The symmetry of the output structure was improved to avoid field distortions which might steer the beam into the microwave circuit. To meet these conditions, XC5 incorporated a disk-loaded traveling-wave output scction, with a $36 \%$ increase in beam-tunnel diameter and dual output waveguides for better output coupling symmetry. The performance of XC5 was a major improvement in output power over earlier tubes in the series. The 50-MW peak power achieved was similar to the previous tubes. However, XC5 operated successfully with $50 \mathrm{MW}$ at $1-\mu$ s pulse length. The tube was opened after a subsequent heater failure and the disk-loaded output circuit showed some signs of overheating, indicating that further refinement of the design still was needed.

XC6 had gun and gain sections similar to XC3 and XC4. However, the output circuit consisted of two uncoupled resonant cavities, each with two output waveguides, resulting in a total of four output windows. The elimination of the inductive coupling iris between the cavities gave XC6 a symmetry not present in any of its four predecessors, as did the use of four symmetrical output waveguides. Dividing the power among four output windows, though not a desirable long-term solution, was intended to allow us to obtain long-pulse, high-power operation with less concern for window failure. XC6 produced 85-MW of peak power at short pulse length. A preliminary look at $0.7-\mu$ s pulse length produced $50-\mathrm{MW}$ of peak power, indicating that the power capability of XC6 was at least as good as XC5. Unfortunately, the gun arced before the tube could be conditioned at higher power and longer pulse length. An attempt to rebuild the tube failed when the output waveguide opened-up while re-baking the tube. Consequently, the power capability of XC6 at longer pulse lengths never was explored.

$\mathrm{XC7}$ was identical to XC5, except for modifications to the disk-loaded travelingwave output section which, in $\mathrm{XC7}$, contained four cavities instead of three. The beam-tunnel diameter in the output section was larger than in XC5 for greater isolation of the microwave circuit from the electron beam, and for lower surface-field gradients. The beam-exit conditions were altered by increasing the pole-piece diameter to overcome the melting of the collector which was observed in XC5. XC7 achieved 
the same peak power as XC5 at short pulse length. However, the pulse length was limited to less than $0.2 \mu \mathrm{s}$ by high field gradients in one cell of the traveling-wave output structure. This condition was later diagnosed by opening the output circuit.

XC8 incorporated a disk-loaded four-cavity resonant output circuit which featured the lowest surface-field gradient of any of the circuits tested. The beam tunnel of the circuit was larger in diameter than any of the previous circuits. In order to compensate for the adverse impact this might otherwise have on efficiency, the XC8 circuit included an improved drive section, incorporating two additional cavities, which were to provide the output circuit with $20 \%$ more rf current than that available in the earlier tubes. Unfortunately, oscillation in a different mode made it impossible to evaluate the rf circuit.

The XC8 output circuit has since been redesigned with only three cavities and will be incorporated in the first klystron (XL1) of the reduced-perveance, 50-MW, XL series. Simulations of the XL1 design have been performed using a two-dimensional particle-in-cell code (CONDOR) assisted by three-dimensional rf-field modeling (by MAFIA). The simulations predict that XL1 will produce $70 \mathrm{MW}$ of output power, and that the tube will not oscillate in the mode that plagued XC8.

\subsubsection{XL-Series Klystrons}

The 50-MW goal for the NLCTA XL tubes considerably eases the design challenges relative to the 100-MW XC series. The XL tube design is based on microperveance of 1.2 ( $350 \mathrm{~A}$ at $440 \mathrm{kV}$ ). The $50-\mathrm{MW}$ goal requires only $33 \%$ beam-to-rf conversion efficiency. However, much greater efficiency and power are expected to be achieved. The plan for developing these tubes is discussed in Scction 3.11.

\subsubsection{RF Windows}

A weakness in the basic design of the XC-series of tubes was that the circular $\mathrm{TE}_{11}$-mode $\mathrm{rf}$ windows used were marginal at the high peak power. The testing of XC5 was interrupted twice by window failures, which required replacement of the windows and reprocessing of the tube. Analysis of the window failures showed that the failures originated at the junction between the window and the waveguide, where the rf electric-field lines terminate.

Consequently, a program was initiated to develop a circular $\mathrm{TE}_{01}$-mode output window. This new window should be free of the previously observed failure mode because, in the $\mathrm{TE}_{01}$-mode, no electric-field lines terminate at the braze junction between the window and the waveguide wall. Thus far in our $\mathrm{TE}_{01}$-mode window development we have achieved $99.5 \%$ mode-purity and a good rf match in setting up the $\mathrm{TE}_{01}$ mode and transmitting it through the circular window. Designs for both the mode transducer and the window have been completed. High-power tests of this new window design in a resonant ring are planned during Summer 1993. Assuming success, it is planned to use $\mathrm{TE}_{01}$ windows on all future high power $\mathrm{X}$-band tubes. 


\subsubsection{Klystron Focusing}

A longer-term problem in developing an economical NLC rf system is reducing the power consumed by the solenoidal electromagnet which focuses the beam in each klystron. We have designed a periodic permanent-magnet (PPM) focusing system which could provide the necessary focusing without consuming power. The PPM focusing system will be tested in a dc beam tester later this year. The rf operation of a PPM-focused klystron has been simulated using the two-dimensional particle-in-cell code, CONDOR. The results are comparable with those obtained from simulations of solenoid focusing. The most successful of the solenoid-focused XL klystrons will be redesigned for PPM focusing in 1994. 


\subsection{ACCelerator Structure}

\subsubsection{Wakefield Suppression}

The NLC design calls for accelerating a train of 90 bunches on each rf pulse in order to achieve the desired luminosity. One limitation of this approach comes from the coupling of the betatron motion of the bunches due to the long-range wakefields generated in the accelerator structures. If not controlled, these wakefields will produce a large growth in the transverse motion of the bunches which will lead to luminosity degradation and possibly beam breakup. To control this growth, the wakefields witnessed by the bunches must be reduced by about two orders of magnitude. Two methods are being considered to achieve this reduction, each of which has been tested experimentally. ${ }^{19,20}$ In the first, the higher-order modes in the structure are damped by coupling them to radial waveguides which are terminated in matched loads. The second technique involves making the higher-order-mode (HOM) frequencies different for each cell while keeping the $\frac{2}{3} \pi$ phase shift in the accelerating mode constant. To the subsequent bunches following behind the driving bunch, the total wakefield, which is the sum of wakefields from the individual cells, decohcres because of the different frequencies of its components. Consequently, the effective wakefield is reduced.

For the NLCTA, we plan to use the detuned structure being developed at SLAC as a prototype for the NLC. ${ }^{21}$ Parameters of this structure are listed in Table 5. Although the NLCTA initially will not have the NLC bunch spacing for which the structure detuning is designed, the wakefield supression still will be effective at preventing beam breakup.

The structure design uses a truncated Gaussian distribution of the HOM frequencies to produce a Gaussian fall-off in the initial time dependence of the sum of wakefields. For this purpose, the relative distribution of HOM frequencies of the 204 cells in a section will have a standard deviation of $2.5 \%$ and will be truncated at $\pm 5 \%$. This distribution will be obtained by modifying the design of a constantgradient section so that the detuning is most pronounced for the cells near the ends of each section. This will result in a structure in which the iris sizes decrease rapidly at the beginning, decrease more slowly in the middle, and decrease rapidly again toward the end of each section.

Figure 8 shows a comparison of the accelerating field obtained for a square input rf pulse of 100-MW amplitude in three different cases: constant impedance, constant gradient, and Gaussian-detuned HOM.

With this distribution of HOMs, the wakefield will decohere to much less than $1 \%$ of its peak value early in the 40 -meter-long bunch train, and then will recohere partially, as illustrated by Figure 9 which was obtained using an equivalent-circuitmodel calculation ${ }^{22}$ that incorporates cell-to-cell coupling.

Simulations of bunch transport in the NLC show that this decoherence is sufficient to control beam breakup if several sets of detuned accelerator sections with 


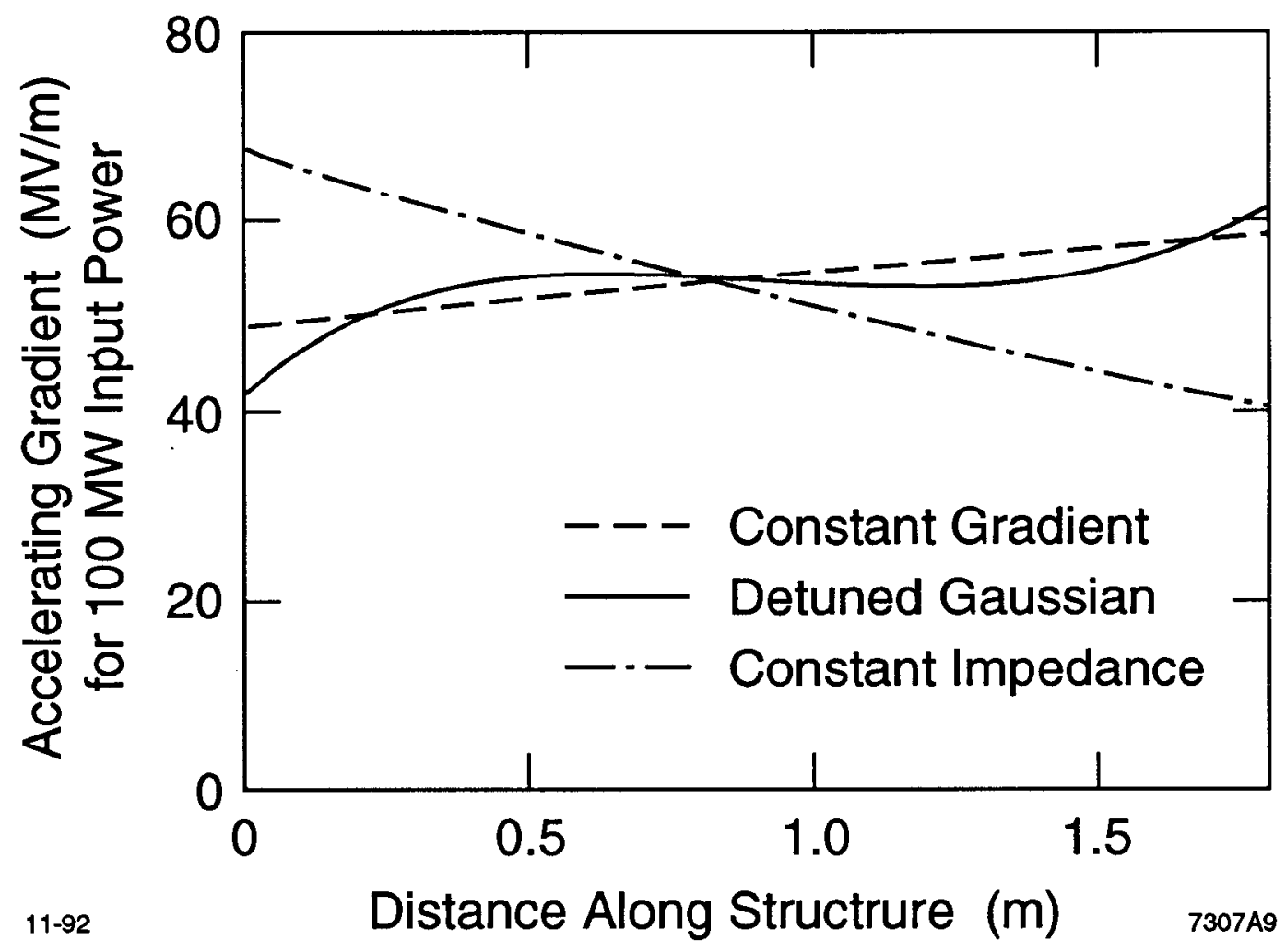

Figure 8. Comparison of accelerating field profiles in a 1.8-m-long accelerator section for 100-MW input power. 
Table 5. NLCTA Structure Parameters

\begin{tabular}{|l|c|}
\hline Section length & $1.8 \mathrm{~m}$ \\
\hline Phase advance per cell & $2 \pi / 3$ \\
\hline Iris aperture radius & $5.72-3.91 \mathrm{~mm}$ \\
\hline Iris aperture normalized radius & $0.218 \lambda-0.149 \lambda$ \\
\hline Group velocity & $0.12 c-0.03 c$ \\
\hline Filling time & $100 \mathrm{~ns}$ \\
\hline Unloaded time constant & $207-186 \mathrm{~ns}$ \\
\hline Attenuation parameter & $0.517 \mathrm{nepers}$ \\
\hline Elastance $(\omega R / Q$ per unit length) & $853-946 \mathrm{~V} / \mathrm{pC} / \mathrm{m}$ \\
\hline Peak input power/(1.8 m) for $50 \mathrm{MV} / \mathrm{m}$ & $48.1 \mathrm{MW} / \mathrm{m}$ \\
\hline Peak power per feed for $50 \mathrm{MV} / \mathrm{m}$ & $86.5 \mathrm{MW}$ \\
\hline $\begin{array}{l}\text { Structure average power dissipation for } 50 \mathrm{MV} / \mathrm{m}, \\
250-\mathrm{ns} \text { pulse length, } 180 \mathrm{pps}\end{array}$ & $1.4 \mathrm{~kW} / \mathrm{m}$ \\
\hline
\end{tabular}

different sets of HOM frequencies, slightly displaced with respect to each other, are used. The implications for bunch transport in the NLCTA are discussed in Section 2.5.

\subsubsection{Field Emission at High Gradients}

Theoretical and experimental studies on the behavior of copper accelerator structures under extremely high rf fields have been carried out at SLAC for several years. ${ }^{23,24}$ The structures which have been examined recently at X-band are a seven-cell standing-wave (SW) section and a 30-cell traveling-wave (TW) section. Both structures are of the constant-impedance uniform-aperture type with a $\frac{2}{3} \pi$ phase shift per cell at $11.424 \mathrm{GHz}$. The maximum surface electric field that was reached on the disks exceeded $500 \mathrm{MV} / \mathrm{m}$ for the seven-cell SW section. The 30-cell TW section was operated stably at an accelerating gradient of $100 \mathrm{MV} / \mathrm{m}$ for an extended period of time. We have studied in considerable detail the problems of $\mathrm{rf}$ breakdown and "dark current" generated by field emission at high gradient. ${ }^{23,24}$ The dark current can absorb rf energy, get accelerated, and produce undesirable steering effects, detrimental x-ray radiation, and experimental-physics backgrounds at the final focus. Many experiments have been done to measure the amplitude and spectrum of the dark current and to study phenomena related to rf breakdown such as outgassing, radiation, heating, etc. We have concluded that the dark current can be minimized by improving surface finish and cleanliness, and by using gas processing and rf processing. Figure 10 shows the dark current measured in a Faraday cup as a function of 


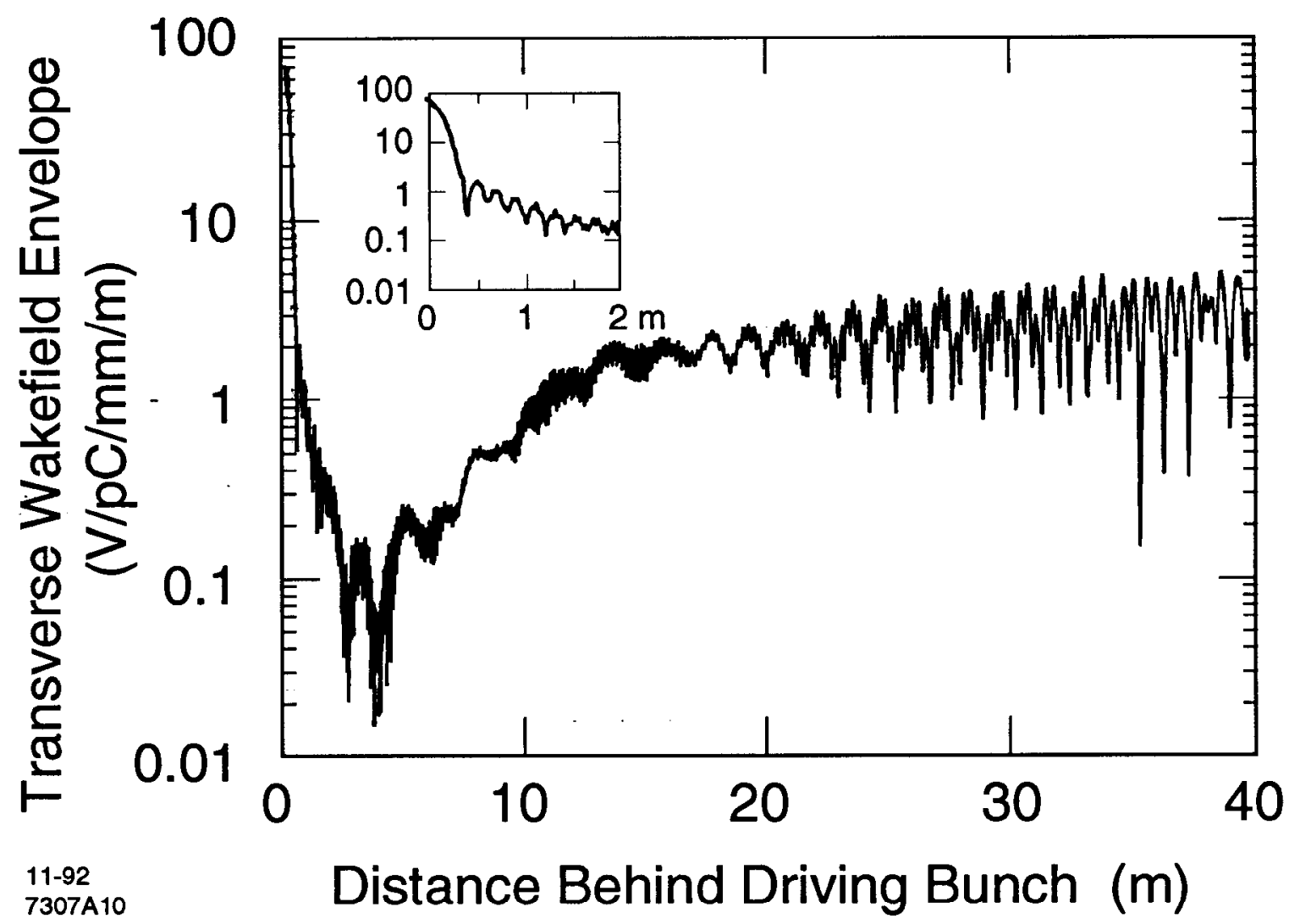

Figure 9. Calculation of the transverse wakefield in an NLCTA accelerator section. (The insert shows the first two meters behind the driving bunch on an expanded distance scale.) 
average accelerating field for three stages of rf processing of the 30-cell TW section. The dark current for an accelerating gradient of $50 \mathrm{MV} / \mathrm{m}$ was found to be negligible. At $100 \mathrm{MV} / \mathrm{m}$ it may be tolerable. We are planning further experimental studies with the full-length NLC-prototype sections in the Klystron Test Laboratory and at the NLCTA. 


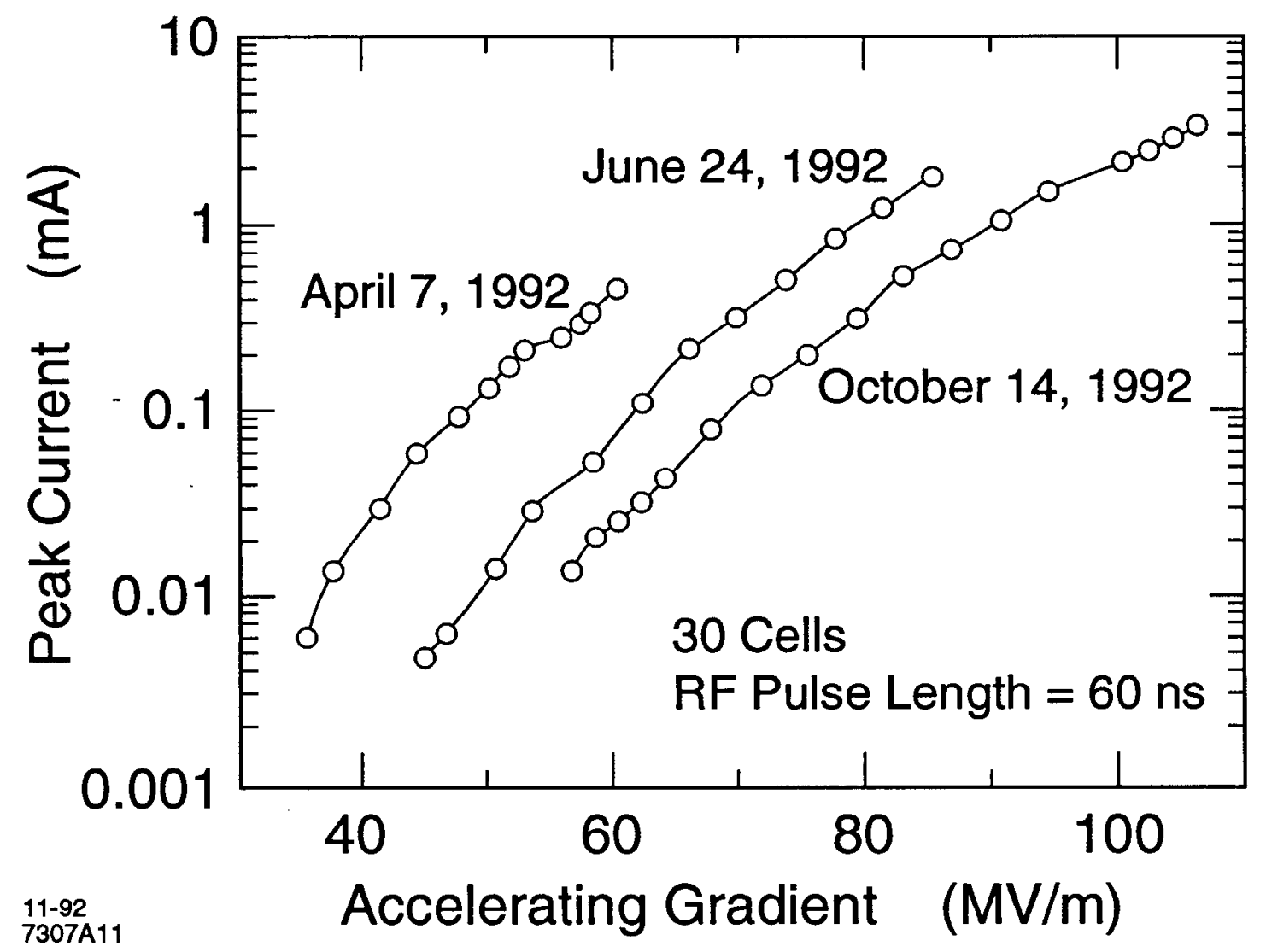

Figure 10. Peak dark current within the pulse measured after three stages of $\mathrm{rf}$ processing of the 30-cell traveling-wave section. 


\subsection{Transverse Wakefield Effects}

We have calculated the multi-bunch beam breakup expected in the NLCTA injector and linac using the methods of Reference 25. The parameters assumed are shown in Table 6. The X-band bunch spacing of the NLCTA design injector was assumed. Since this spacing is shorter than the decoherence length of the wakefield, the results of the calculation represent a worse case, compared to an NLC-like bunch spacing. The injector will have solenoidal focusing, so the $\beta$-function there was assumed to be proportional to the beam energy $(E)$. The $\beta$-function in the linac was assumed to be constant at $5 \mathrm{~m}$. A beam loss of $20 \%$ was assumed between the injector and the linac.

In Figure 11(a), we show the factor by which the transverse oscillation amplitude of each bunch has blown up by the end of the injector, assuming that all bunches are injected with the same initial transverse offset, and assuming that the cell-tocell transverse-mode frequency-detuning distribution of the injector preacceleration section is Gaussian over $\pm 2 \sigma$ with $\sigma=2.5 \%$ (as discussed in Section 2.4). The corresponding result if the injector section were not detuncd is shown in Figure 11(b). The situation is improved significantly by detuning, as expected. The results of the beam-breakup calculation should be interpreted with some caution because the calculation assumes a fully relativistic beam, which is not the case at the beginning of the injector section, and because the calculation averages the effect of each of the detuned synchronous modes over the whole section, while in reality there are many betatron oscillations within the injector section. We do not expect either of these simplifications to dramatically change the result that the blowup is small in a detuned injector section. However, the actual beam breakup is unlikely to be quite as small as that seen in Figure 11(a).

In Figure 12, we show the factor by which the transverse oscillation amplitude of each bunch has blown up by the end of the detuned linac. Detuning the 1.8-m linac sections is essential for controlling the multi-bunch beam breakup; without detuning, the beam breakup is worse by many orders of magnitude. Since the bunches from the injector are hardly blown up at all (assuming detuning), we have assumed for simplicity that the bunches entering the linac all have the same initial amplitude and phase. In addition to the nominal detuncd cell frequency distribution, we have included the effects of random errors in the cell frequencies, with relative errors normally distributed with standard deviation $10^{-4}$. The frequency errors have been assumed to be systematic, i.e. the same for all the sections, which is the most pessimistic case.

Figure 12 shows a very small amplification of an initial transverse beam offset in the NLCTA. This amplification is similar to that obtained in NLC simulations. The NLCTA, in spite of its short length, is quite sensitive to transverse wakefields because of its very low beam energy. 


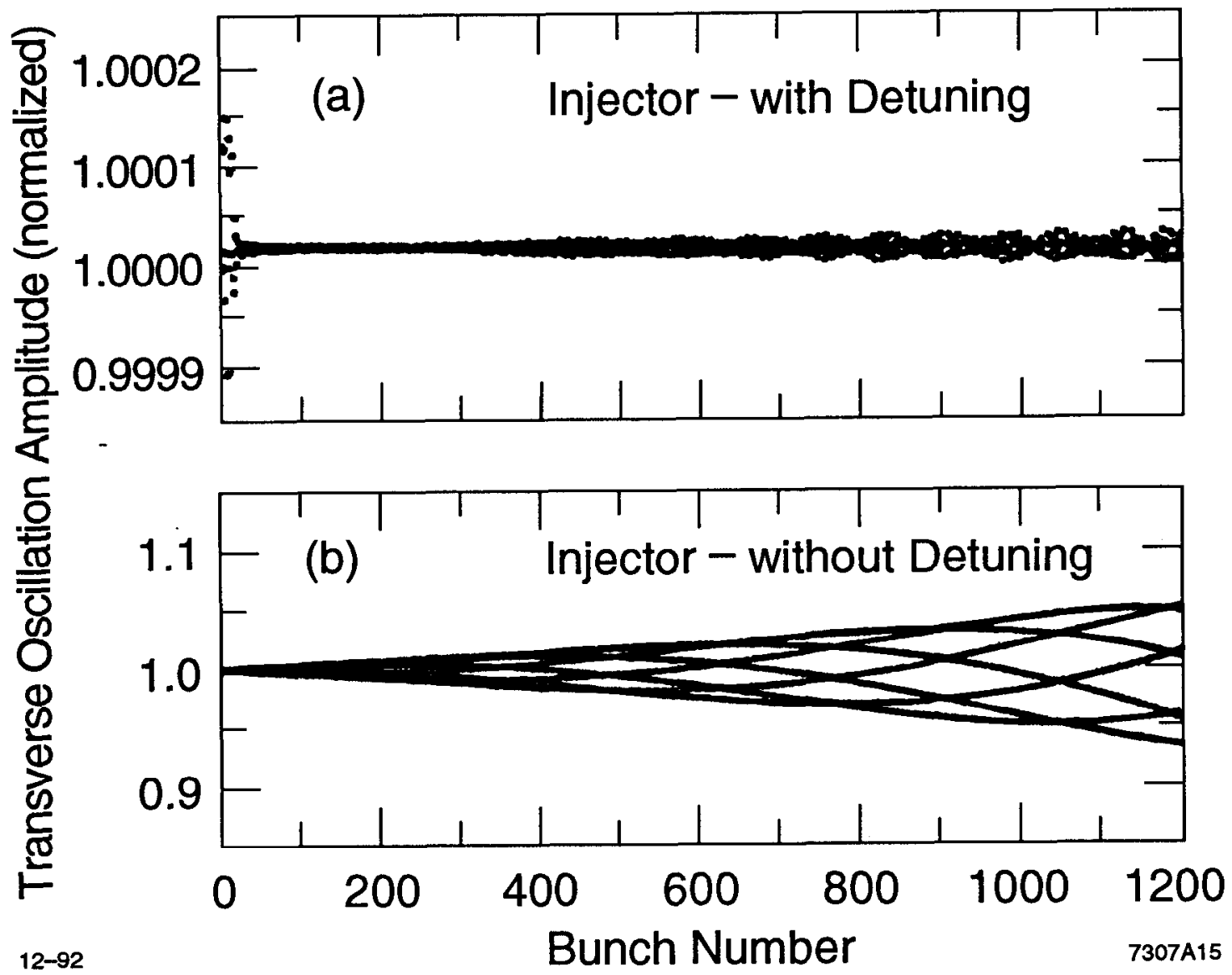

Figure 11. Bunch transverse oscillation amplitudes at end of injector, with and without detuning. 


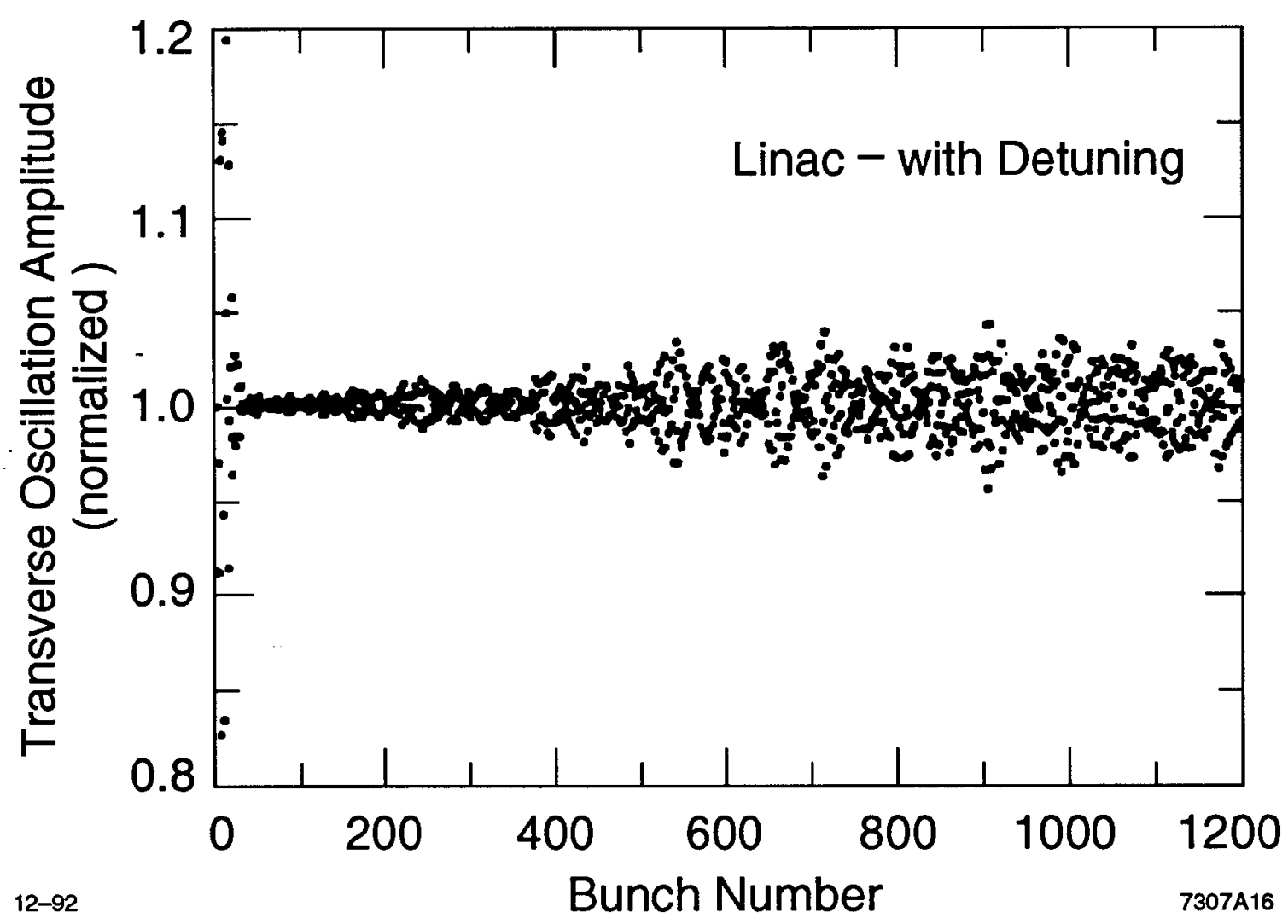

Figure 12. Bunch transverse oscillation amplitudes at end of linac, with detuning. 
Table 6. Assumptions for the beam-breakup calculation

\begin{tabular}{|l|c|c|}
\hline & Injector & Linac \\
\hline Initial energy & $0.200 \mathrm{MeV}$ & $86 \mathrm{MeV}$ \\
Final energy & $86 \mathrm{MeV}$ & $500 \mathrm{MeV}$ \\
Electrons per bunch & $5 \times 10^{8}$ & $4 \times 10^{8}$ \\
$\beta$-function & $7 \times 10^{-4} \mathrm{~m} \times E /(200 \mathrm{keV})$ & $5 \mathrm{~m}$ \\
Length & $1.8 \mathrm{~m}$ & $12 \mathrm{~m}$ \\
Bunch spacing & $2.624 \mathrm{~cm}$ & $2.624 \mathrm{~cm}$ \\
\hline
\end{tabular}




\subsection{Beam-Loading Compensation}

The NLC bunch train is currently expected to be close to one filling time in length, with $0.65 \times 10^{10}$ electrons per bunch. The relative energy uniformity desired over the bunch train is $10^{-3}$. However, the beam loading, if not compensated, will reach a steady-state value of $25 \%$ by the end of the bunch train. The most promising beam-loading energy-compensation strategy for bunch trains of this length is to prefill the structure with $\mathrm{rf}$ in such a way that the energy gain of each bunch during the transient period approximates the energy gain of each bunch in the steady state. In one implementation of this scheme, the rf pulse is modulated so that the rf electricfield envelope at the input-end of the structure is ramped linearly during one filling time before the bunch train is injected. Since dispersion in the accelerator structure creates large transients on the leading edge of the rf pulse, it is desirable to wait an additional several nanoseconds before injecting the bunch train, to allow the worst of these transients to propagate out of the structure.

A frequency-domain analysis of the above beam-loading compensation scheme is shown in Figure 13, where a 1.8-meter-long accelerating section of the NLCTA is modeled as a constant-gradient structure with filling time of $100 \mathrm{~ns}$, attenuation of 0.517 nepēr, average elastance ${ }^{*}$ of $900 \mathrm{~V} / \mathrm{pC} / \mathrm{m}$, and unloaded gradient of $50 \mathrm{MV} / \mathrm{m}$. For the simulation illustrated, a train of bunches, spaced by $1.4 \mathrm{~ns}$ ( $16 \mathrm{rf}$ periods) and containing $0.7 \times 10^{10}$ electrons per bunch, enters the structure $104 \mathrm{~ns}$ after the leading edge of the rf. In Figure 13, the loadcd and unloaded energy gain of a test charge is plotted as a function of the time (relative to the leading edge of the rf pulse) the test charge is injected into the structure. The figure shows that the desired compensation to $10^{-3}$ is achieved in this simulation. Since the detuned structures of the NLCTA are only approximately constant-gradient, it may be desirable or necessary to employ modulation schemes that differ slightly from the linear ramp in rf electric-field envelope to improve the beam-loading energy compensation.

If the bunch train is shorter than about half the filling time, then a "matched filling" technique can be used. In this technique, the bunches are injected before the structure is completely filled, so that the unloaded increase in the accelerating voltage between the arrival times of the bunches approximately cancels the beam loading. One may further improve the compensation by modulating the rf input during the time when the train is passing through the structure, to compensate the small droop in energy that one would otherwise get in the middle of the bunch train.

The initial version of the NLCTA injector will provide a train of bunches with $\frac{1}{16}$ the bunch spacing and $\frac{1}{16}$ the bunch charge of the NLC bunch train. Since the beam-loading depends mainly on the average current, it will be possible to test beamloading compensation techniques relevant for the NLC with the NLCTA bunch train.

* Elastance is defined as $\omega R / Q$ per unit length. 

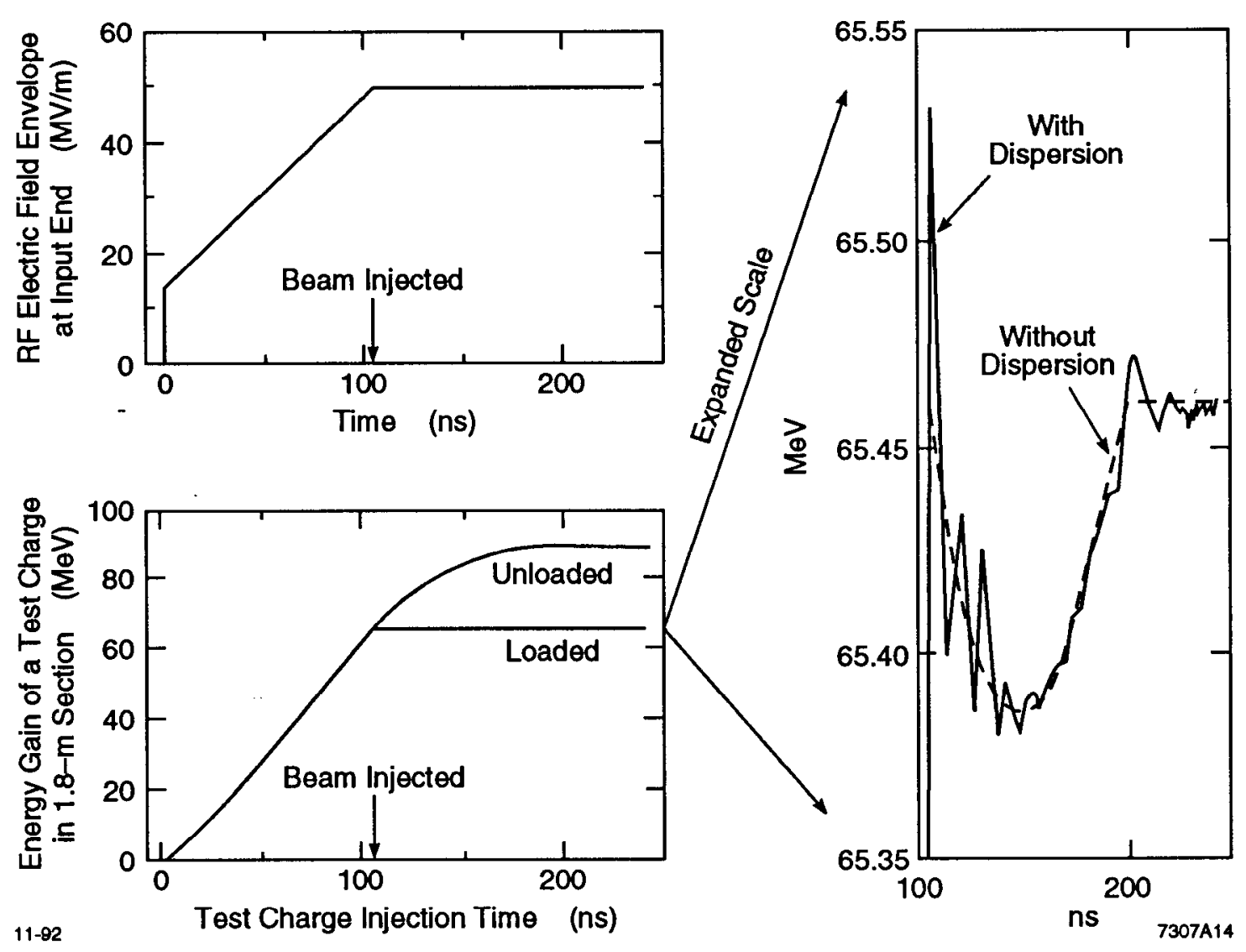

Figure 13. Frequency-domain analysis of a possible beam-loading compensation scheme. 


\subsection{INJECTOR}

The design of the NLCTA injector is intended to be the simplest one capable of testing the performance of the NLCTA linac and rf system, particularly with regard to evaluating multi-bunch beam-loading compensation. The design goal is to employ in the injector only very simple, stable, and well-tested technology with outstanding reliability so that the injector would be a well understood tool, rather than a subject of experimentation. Consistent with the goal of simplicity, the bunch spacing will be at the 88-ps period of the accelerating frequency, which is $\frac{1}{16}$ of the NIC design bunch spacing. However, the average current in the NLCTA bunch train will be identical the NLC design so the multi-bunch beam loading in the NLCTA will be nearly identical to the NLC.

The NLCTA injector design comprises the following components:

1. A thermionic-cathode gun with a grid for controlling current and pulse length.

2. An X-band velocity modulation prebuncher followed by a drift in which the bunching occurs.

3. A 0.9-meter-long buncher/capture accelerator section.

4. A second 0.9-meter-long preacceleration section that raises the energy from 45 $\mathrm{MeV}$ to $90 \mathrm{MeV}$.

5. A focusing solenoid which surrounds all the above except the gurl.

6. A bucking coil which reduces the magnetic field at the cathode to zero.

The NLCTA injector specifications are listed in Table 7.

The NLCTA injector design incorporates two 0.9 -meter-long accelerator sections rather than one of the standard 1.8-meter-long structures for two reasons: (1) The phase of the second section can be adjusted for optimum energy spread within the individual bunches. (2) The beam-loading coefficient is reduced so that the same beam-loading compensation used in the rest of the accelerator is viable for higher currents in the injector. This allows the NLCTA injector to deliver currents that exceed the NLC design specification so that it can deliver the design current after collimation in longitudinal and transverse phase space. (Approximately one-third of injector current is expected to be lost by collimation.)

The injection energy is determined by the requirement of small transverse and longitudinal emittance at the entrance to the linac. Small transverse emittance is required for good beam transport through the small-aperture accelerator sections. Small longitudinal emittance is required in order to meet the bunch-length and energyspread requirements.

In the future, we plan to upgrade the injector to produce a train of bunches similar to that required for the NLC in order to carry out more detailed beamdynamics studies. Possible specifications for such an upgrade are given in the last 
Table 7. NLCTA Injector Parameters

\begin{tabular}{|l|c|c|}
\hline \multicolumn{1}{|c|}{ Parameter } & $\begin{array}{c}\text { NLCTA } \\
\text { Design }\end{array}$ & $\begin{array}{c}\text { Injector } \\
\text { Upgrade }\end{array}$ \\
\hline Gun voltage (kV) & 150 & \\
Gun current (A) & 2 & \\
Pulse length (ns) & $1-140$ & $1-140$ \\
Injector unloaded energy gain (MeV) & 90 & 90 \\
Normalized emittance (rad-m) & $<10^{-4}$ & $<10^{-5}$ \\
RMS bunch length (mm) & 0.4 & 0.2 \\
RMS energy spread (\%) & 0.5 & 0.2 \\
Bunch frequency (GHz) & 11.424 & 0.714 \\
Bunches per pulse & $10-1600$ & $1-100$ \\
Electrons per bunch* & $0.4 \times 10^{9}$ & $0.7 \times 10^{10}$ \\
Electrons per pulse* & $0.7 \times 10^{12}$ & $0.7 \times 10^{12}$ \\
Pulse repetition rate (pps) & 10 & 10 \\
\hline
\end{tabular}

*After collimation in the chicane.

column of Table 7. In order to produce the multi-bunch beam, the upgraded injector may use a thermionic-cathode gun with a pulsed grid ${ }^{26}$ and subharmonic bunchers. If lower emittance is required, it may be necessary to use a photocathode ${ }^{27}$ in an rf gun. ${ }^{28}$ The impact of an injector upgrade on the experimental program is discussed in Section 4.7. 


\subsection{Chicane and Linac Beam Transport}

Following the injector will be a pair of "sieve" collimators that will be used to adjust the intensity and maximum transverse size of the bunches. The intensity attenuation capability will allow the optimization of the beam-loading compensation in the injector and linac to be dccoupled: i.e., once the injector has been set up, the linac intensity then can be adjusted with the sieve, if necessary, to allow for optimum beam-loading compensation without having to re-tune the injector.

To adjust the longitudinal phase space of the bunches, a chicane incorporating four bends will follow the sieve collimators. A collimator with adjustable jaws will be located at a high-dispersion point near the center of the chicane so that the low-energy tail of the bunches can be clipped off. The low-energy tail is due to the combination of the exponentially-shaped longitudinal bunch profile which results from the bunching process, and the subsequent acceleration in the sinusoidal rf field which correlates the longitudinal position and energy of the electrons in the bunches. Hence, cutting off the low-energy tail will shorten the bunch length. To do the collimation, the chicane optics were designed to make the transverse size of the dispersed bunches much larger than that of monochromatic bunches. A horizontal dispersion $\left(\eta_{x}\right)$ of $35 \mathrm{~cm}$ was chosen for the center of the chicane, where $\beta_{x}$ and $\beta_{y}$ were constrained to be $1 \mathrm{~m}$. At this point, the undispersed rms transverse size of the beam is $\sigma=0.42 \mathrm{~mm}$ and the transverse size due to dispersion ( $\eta$ times the rms energy spread) is $8.4 \sigma$ for a $1 \%$ rms energy spread.

For a given collimator setting, we will be able to trade-off energy spread and bunch length by changing the rf phase in the second $0.9-\mathrm{m}$-long accelerator section in the injector to achieve the desircd energy spread, and then changing the chicane bunch compression (which is represented by the $R_{56}$ element ${ }^{*}$ of the TRANSPORT matrix) to achieve the corresponding minimum bunch length. For such changes we would like the rms energy spread of the bunches to be less than $1 \%$ so that the emittance blowup in the chicane due to chromaticity and second-order dispersion is not too large (less than 15\%). We would also like the intra-bunch energy spread in the spectrometer at the end of the linac to be less than $1 \%$ to make it easier to discern bunch-to-bunch energy differences down to the $0.1 \%$ level for the multi-bunch beamloading compensation studies. Achieving this energy spread in the spectrometer will require that the bunch length in the linac be less than $0.5 \mathrm{~mm}$.

However, the price paid for bunch compression is greater than just the increased intra-bunch energy spread in the chicane. The chicane, when set for bunch compression $\left(R_{56} \neq 0\right)$, will convert bunch-to-bunch energy differences from the injector into bunch-to-bunch phase differences with respect to the accelerating wave, which will then be converted into energy differences in the linac. These energy differences will make it harder for us to study beam-loading compensation. Fortunately, we can

* The path-length difference for two relativistic particles is $R_{56}$ times their relative energy difference. 
make the chicane isochronous $\left(R_{56}=0\right)$ to avoid this problem, and we can still meet the longitudinal phase-space requirements. One way to do this is to set the chicane collimator to limit the full bunch length to $1.5 \mathrm{~mm}$, which will transmit about $70 \%$ of the electrons, and will result in an rms bunch length of $0.4 \mathrm{~mm}$ and a minimum rms energy spread of about $0.5 \%$. The plan is to operate with these parameters although we have allowed enough flexibility in the design of the chicane so that, if the bunch-to-bunch energy differences can be tolerated, the quadrupole settings in the chicane can be adjusted to give the desired bunch compression. For example, the bunch length can be halved to $0.2 \mathrm{~mm}$ with $R_{56}=-10 \mathrm{~mm}$; however, in this case the energy spread in the chicane would double to $1 \%$.

To achieve isochronicity $\left(R_{56}=0\right)$, the chicane lattice was designed ${ }^{29}$ so that the phase advance between adjacent bends is $180^{\circ}$, and hence the dispersion goes though zero at each of the bends. This requires a rather large total phase advance, and therefore a large number of quads. In addition, the spacing between quads must be fairly large to prevent rapid changes in the $\beta$-function, and to accommodate beamdiagnostic instrumentation. The resulting design has a total of 12 quads, each $15 \mathrm{~cm}$ long, spaced over a distance of $5.4 \mathrm{~m}$ between the first and last bend. Also, four quads are used upstream of the chicane to match from the optics of the injector, where it is assumed that $\beta_{x}=\beta_{y}=0.35 \mathrm{~m}$ and $\alpha_{x}=\alpha_{y}=0$ at the exit of the injector solenoids. Likewise, four quads are used to match to the linac optics. Specific sets of quad settings have been computed which achieve values of $R_{56}$ in the range from $-7.5 \mathrm{~cm}$ to $+2.5 \mathrm{~cm}$, including the isochronous case where $R_{56}=0$.

A study of the sensitivity of the $R_{56}$ matrix element of the chicane to errors in the magnetic field strengths was done using the program DIMAD. Errors were introduced by changing the strength of individual quads and bends by $1 \%$ in the DIMAD input describing the chicane. The isochronicity is rather well preserved; the $R_{56}$ variation resulting from each error that was introduced is less than $1.5 \mathrm{~mm}$, which is small compared to the $10-\mathrm{mm}$ tolerance on $R_{56}$.

The linac optics were designed ${ }^{29,30}$ to keep the transverse beam size small compared to the apertures of the accelerator structures, which vary from $5.7 \mathrm{~mm}$ to $3.9 \mathrm{~mm}$ in radius. A constraint in reducing the $\beta$-function for this purpose is the $1.8-\mathrm{m}$ length of the accelerator structures since the quads will only be placed between the structures. A FODO lattice with about $100^{\circ}$ of phase advance per cell was chosen which yields a maximum $\beta$ of approximately $8 \mathrm{~m}$ and a worst-case beam stay-clear of $5 \sigma$. This lattice design also takes into account the rapid beam-energy gain along the linac, which is expected to be $50 \mathrm{MeV} / \mathrm{m}$ (unloaded) for the initial running.

The lattice functions and the rms beam size for the isochronous chicane and linac are shown in Figure 14. The functions were calculated and cross-checked with the computer codes, DIMAD, COMFORT, and TRANSPORT. 

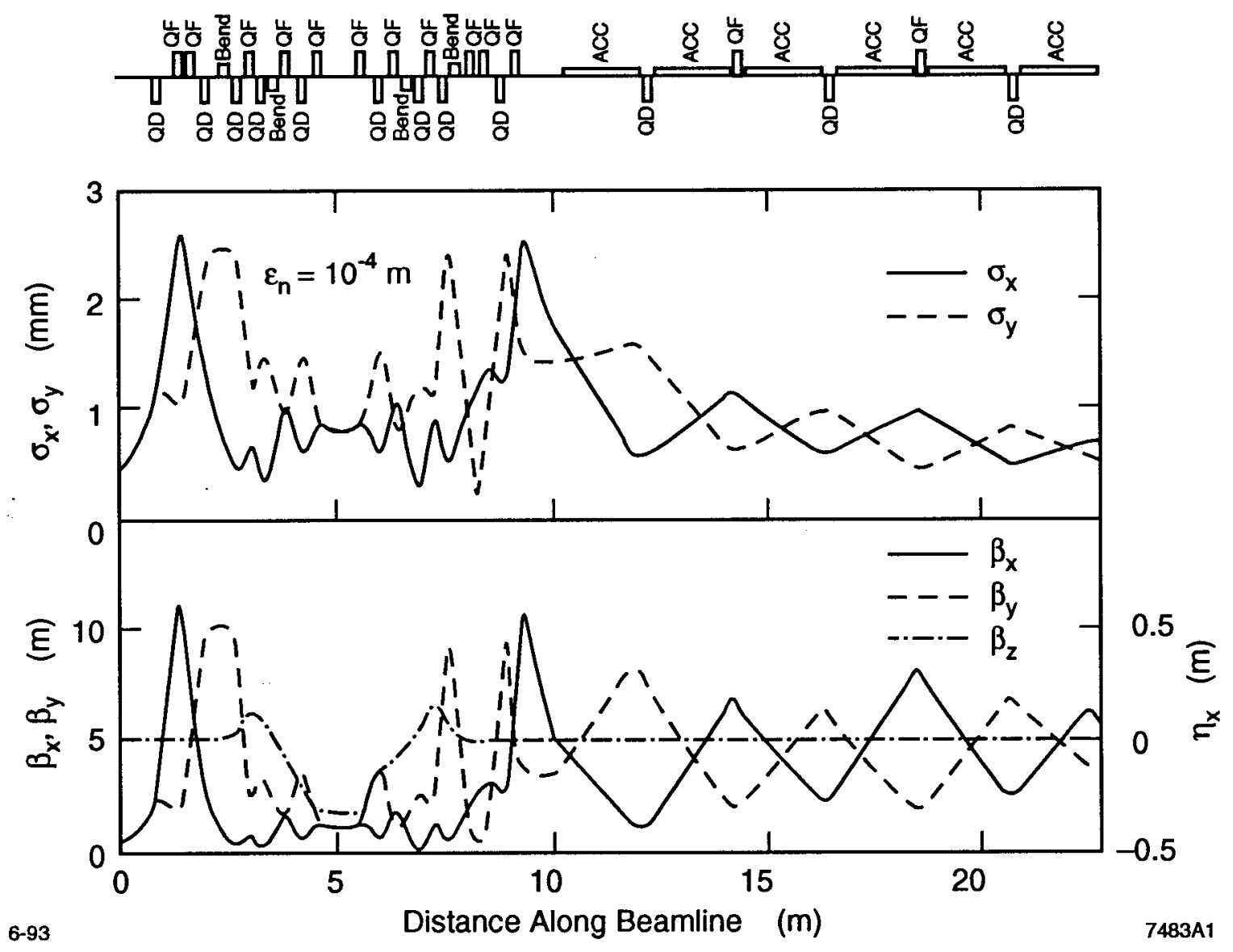

Figure 14. Beam size and lattice functions along the isochronous chicane and linac. 


\subsection{SPECTROMETER}

A spectrometer will follow the linac and be used to measure the energy and transverse phase-space of the bunch train. The spectrometer will utilize an existing dipole " $\mathrm{H}$ "-magnet to steer the beam by $12^{\circ}$ into a high-dispersion line. When the magnet is not powered, the beam will enter a straight-ahead line where the undispersed bunch size can be measured. The aperture of the straight-ahead line will be made large enough to allow coarse energy measurements with the spectrometer magnet partially powered. Both the straight-ahead and high-dispersion line will terminate in heavily-shielded beam dumps.

Figure 15 shows the spectrometer lattice that will follow directly after the last linac section. This double focus optics has a focal point before the bend magnet (at PM1 in the figure, where PM refers to Profile Monitor) and one after it, at the end of the $12^{\circ}$ line (PM2 in the figure). Four quadrupoles are used to match the incoming beam to symmetric double waists at PM1 where $\beta_{x}=\beta_{y}=0.22 \mathrm{~m}$, and a triplet of quadrupoles is used to symmetrically refocus the beam at PM2 where $\beta_{x}=\beta_{y}=0.55 \mathrm{~m}$.

At PM2, where the horizontal dispersion, $\eta_{x}$, is $0.5 \mathrm{~m}$, the first-order resolving power, $R P_{1}$, is

$$
R P_{1}=\frac{\eta_{x}}{\sqrt{\beta_{x} \epsilon_{x}}}=2.3 \times 10^{3}\left(\frac{E}{626 \mathrm{MeV}}\right)^{1 / 2}\left(\frac{10^{-4} \mathrm{~m}-\mathrm{rad}}{\epsilon_{n}}\right)^{1 / 2}
$$

For the expected bunch energy spread of $0.5 \%$, the horizontally dispersed bunch size is about 12 times larger than the undispersed size, which is comfortably larger than that needed for the energy and energy spread measurements.

A pulsed dipole kicker magnet (VKICK in Figurc 15) will separate the bunches vertically so the variation of the of the bunch energy and energy spread within the bunch train can be measured. The $R_{34}$ transport element from the kicker magnet to PM2 is $2.5 \mathrm{~m}$, so the magnet's angular kick of $8 \mathrm{mrad}$ at the highest beam energy of $1.2 \mathrm{GeV} / \mathrm{c}$ will vertically displace the head and tail of the bunch train by $2 \mathrm{~cm}$ at PM2 when the kicker rise time and timing is matched to the bunch train length and transit time. This displacement will be centered about the beam axis using a vertical corrector magnet (YCOR in the figure). The expected energy and time resolution of this system is discussed in Section 3.7.2. 

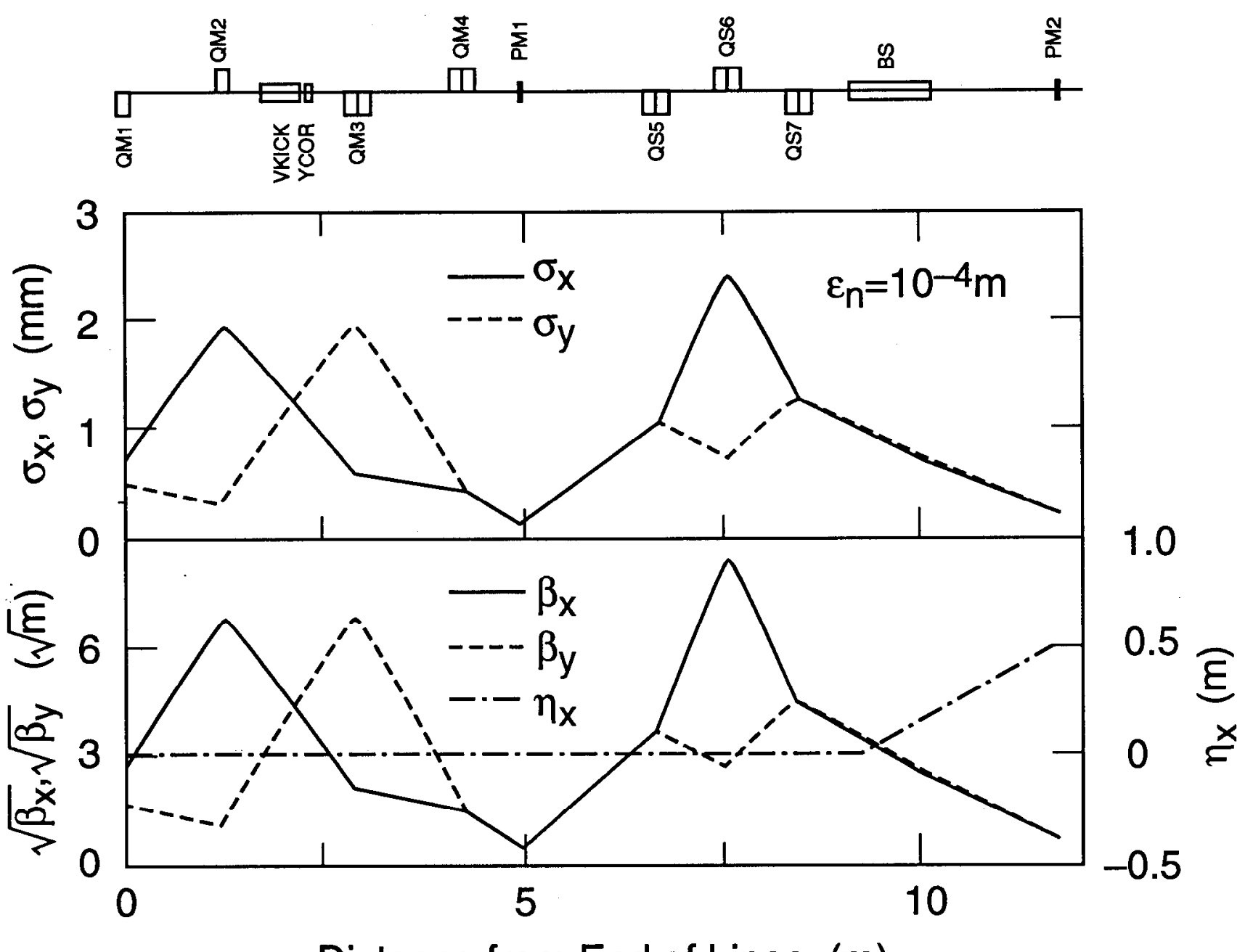

$6-93$

Distance from End of Linac (m)

Figure 15. Beta-functions and rms beam size in the spectrometer. 


\subsection{Radiation ShiElding}

The radiation shielding shown in Figures 2 and 4 is designed to provide adequate protection for personnel working in and around End Station B during NLCTA operations. Specifically, the shielding is designed to limit the average dose-equivalent to occupied areas outside the shield to a maximum of 1 rem per year for 1000 hours per year of 10-pps operation with a beam of the highest possible energy. This level of protection will permit access to the klystrons, modulators, power supplies, and instrumentation racks inside End Station B while the NLCTA is running beam.

The NLCTA injector is designed to be capable of producing a multi-bunch beam of $10^{12}$ electrons per pulse at a beam-pulse repetition rate of $10 \mathrm{pps}$. The rf may be pulsed at up to 180 pps. However, redundant electronic devices will be employed to prevent an unintended radiation dose from beam repetition rates in excess of 10 pps. A collimator halfway down the chicane is assumed to deplete $30 \%$ of the beam current. An insertable Faraday Cup at the end of the chicane, when it is inserted, is assumed to stop the remaining $70 \%$ of the beam. The linac gradient, after an rf system upgrade that quadruples the rf power of the initial design, will be $100 \mathrm{MeV} / \mathrm{m}$, giving a maximum energy gain of $1080 \mathrm{MeV}$. Since the injector design energy is 90 $\mathrm{MeV}$, the full beam energy after the upgrade would be $1170 \mathrm{MeV}$. It is for this case that the radiation shielding has been designed.

The adequacy of the shielding design has been determined by calculating expected dose rates outside the shield for the following representative scenarios:

1. Loss of $30 \%$ of the $90-\mathrm{MeV}$ beam at the chicane collimator.

2. Dumping the collimated $90-\mathrm{MeV}$ beam into the chicane Faraday Cup.

3. Loss of $0.5 \%$ of the $1170-\mathrm{MeV}$ beam (or $9 \%$ of the $630-\mathrm{MeV}$ beam) into the high-energy end of the linac accelerator structure at one point.

4. Dumping the $1170-\mathrm{MeV}$ linac beam into the beam dump.

For the third scenario, the accelerator structure is modeled as a copper slug target, $25 \mathrm{~cm}$ (17 radiation lengths) deep and $8 \mathrm{~cm}$ (6 radiation lengths) in diameter. In all scenarios, neutron attenuation in the target is ignored in the interest of conservatism, giving an estimate of the dose-rate that is exaggerated by approximately $20 \%$. The method of calculation is based on simple physical extrapolations of experimentally determined neutron and photon yields, ${ }^{31}$ and has been used successfully for shielding design at SLAC for 30 years. For the range of energies and shield thickncsses encountered in the NLCTA design, the maximum uncertainty is approximately $\pm 20 \%$ for the high-energy neutron dose that dominates the radiation at $80-90^{\circ}$ from the beam direction. The dose-rate calculations for the above scenarios, including the assumptions made about beam energy and power, are summarized in Table 9.

The calculated dose rates at the sides of the shielding enclosure imply that the shielding should be adequate to permit access to the klystrons, modulators, power 
Table 9. Dose-rate calculations at the surface of the shielding for 10-pps operation with beam

\begin{tabular}{|c|c|c|c|c|c|c|c|c|}
\hline Source & $\begin{array}{c}\text { Direc- } \\
\text { tion }\end{array}$ & $\begin{array}{l}\text { Iron } \\
\text { Thick- } \\
\text { ness }\end{array}$ & $\begin{array}{l}\text { Concrete } \\
\text { Thick- } \\
\text { ness }\end{array}$ & $\begin{array}{c}\text { Elec- } \\
\text { trons } \\
\left(10^{12}\right)\end{array}$ & $\begin{array}{l}\text { Beam } \\
\text { Energy } \\
(\mathrm{MeV})\end{array}$ & $\begin{array}{c}\text { Beam } \\
\text { Power } \\
\text { (W) }\end{array}$ & $\begin{array}{l}\text { Point } \\
\text { Loss } \\
\text { (W) }\end{array}$ & $\begin{array}{c}\text { Dose- } \\
\text { Equiv. } \\
\text { (mrem/h) }\end{array}$ \\
\hline \multirow[t]{2}{*}{ Collimator } & Side & - & $6^{\prime}$ & 1.0 & 90 & 144 & 43 & 0.35 \\
\hline & Roof & - & $4^{\prime}$ & $"$ & $"$ & $"$ & $"$ & 2.4 \\
\hline \multirow{2}{*}{$\begin{array}{c}\text { Faraday } \\
\text { Cup }\end{array}$} & Side & - & 6 & 0.7 & 90 & 101 & 101 & 0.79 \\
\hline & Roof & - & $4^{\prime}$ & $"$ & $"$ & $"$ & $"$ & 5.6 \\
\hline \multirow[t]{4}{*}{ Linac } & Side & - & $6^{\prime}$ & 0.7 & 630 & 706 & 62 & 1.0 \\
\hline & Side & - & 6 ' & 0.7 & 1170 & 1310 & 6.6 & 1.0 \\
\hline & Roof & - & $4^{\prime}$ & $"$ & $"$ & $"$ & $"$ & 3.0 \\
\hline & $\begin{array}{l}\text { Roof } \\
\text { pene- } \\
\text { tration }\end{array}$ & - & $4^{\prime}$ & $"$ & $"$ & $"$ & $"$ & 10.7 \\
\hline \multirow{3}{*}{$\begin{array}{c}\text { Dump } \\
-\end{array}$} & Forward & $3^{\prime}$ & $10^{\prime}$ & 0.7 & 1170 & 1310 & 1310 & 0.42 \\
\hline & Side & $3^{\prime}$ & $6^{\prime}$ & $"$ & $"$ & $"$ & $"$ & 0.96 \\
\hline & & $3^{\prime}$ & $4^{\prime}$ & $"$ & $"$ & $"$ & $"$ & 6.4 \\
\hline
\end{tabular}

supplies, and instrumentation racks inside End Station B while the NLCTA is running beam. The dose rates in these occupied areas do not exceed $1 \mathrm{mrem} / \mathrm{h}$. The dose-rate exceeds $5 \mathrm{mrem} / \mathrm{h}$ at the unoccupied roof areas over the chicane Faraday Cup, near the linac roof penetrations, and over the dump. If the measured dose rates at these areas actually exceed $5 \mathrm{mrem} / \mathrm{h}$, and if local shielding cannot reduce them to below $5 \mathrm{mrem} / \mathrm{h}$, then these areas can be cordoned-off as "radiation areas".

The NLCTA shielding design is quite conservative. Table 9 demonstrates that the shielding design permits up to $0.5 \%$ of the $1170-\mathrm{MeV}$ beam, or up to $9 \%$ of the 630 $\mathrm{MeV}$ beam, to be dumped into the accelerator structure (or a beam-line component) at one point for 1000 hours per year. However, steady-state point losses this large beyond the first accelerator section are unlikely. Such large point losses are possible, and tolerable, for the relatively short periods of time it takes to diagnose them, to limit the pulse repetition rate if necessary, and to reduce the losses to acceptable steady-state levels. The integral dose to an occupied area for one year of operation is expected to be much less than $1 \mathrm{rem}$. 


\section{Accelerator Components}

\subsection{OVERVIEW}

The NLCTA and its auxiliary equipment will occupy approximately one third of the floor area of End Station B (ESB). A plan view of the layout is shown in Figure 2 (of Section 2.1). The layout of the beam line components is shown in detail in SLAC Drawing GP-290-004-04 E0, "NLC Test Accelerator Beamline Installation Master Layout." In the following sections, we describe the various technical components of the NLCTA. Budgetary considerations are discussed for some items. Estimated costs for all items will be covered in Section 5.1.

\subsection{Conventional Facilities}

\subsubsection{ESB Refurbish and Preparation}

End Station B is currently occupied by Beam Lines 19 and 20, and is used to store magnets, detectors, and other valuable material that cannot be left out-of-doors. Most of the stored items, including the Crystal Ball Detector, will have to be moved elsewhere. Beam Line 20, which is closest to the NLCTA shielded enclosure, will be surveyed, then disassembled and stored. It may be reassembled in the next few years, so its footprint will be preserved.

Buildings 404 and 420 will be moved to make room for the extension of the NLCTA beam line outside the east wall of ESB. Building 404 will be moved to the yard area near Beam Dump East where it will house the Crystal Ball Detector and some of its associated equipment. Building 420 will probably be located there also. In addition, Power Supply Shelter 309 will be moved across the road to ease access to the construction area.

\subsubsection{Shielded Enclosure and Dump}

The NLCTA beam line will be housed in a concrete enclosure for the purpose of radiation shielding. The inner dimensions of the enclosure will be 9 feet wide by 10 feet high, except at the downstream end, where it will be 15 feet wide to accommodate the $12^{\circ}$ spectrometer line. The walls will be 6 feet thick and the roof will be 4 feet thick. The walls will be assembled from two sizes of reinforced concrete block which will interlock to prevent line-of-sight passages to the beam line. The roof blocks will also interlock for the same purpose. The dump region will contain steel and additional concrete to meet the radiation shielding requirements. The shielding material will also be used to construct a maze-like entryway at each end of the beam line.

The housing assembly will include earthquake bracing. The wall blocks will sit on 4-inch by 4-inch steel tubes anchored every 10 inches in the concrete floor. This assembly will prevent the wall blocks from sliding horizontally. Steel tubes will 
also lie in grooves between the tops of the wall blocks and the bottoms of the roof blocks to prevent the roof blocks from sliding. The joint between the roof and wall blocks will be strengthened with welded steel angle, alleviating the need for extended side supports. The housing construction technique has been approved by the SLAC Earthquake Safety Committee.

After assembly, any gaps between the shielding blocks will be sealed and the interior of the structure will be painted. The inner surfaces of the wall blocks will contain Unistrut inserts which will be used to anchor cable trays, lights, plumbing, and the sprinkler system. None of these items will be attached to the roof blocks to make it simpler to remove the blocks. The utility tunnel that lies under the enclosure near the beam dump will be sealed with reinforced concrete to reduce radiation levels in the tunnel.

\subsubsection{Control Room}

The accelerator will be operated from a control room located in Building $203 / 236$, south of ESB. This building is next to an access hole to the utility tunnel that runs under ESB, near the upstream end of the shielded enclosure. Since all cabling and utilities will be routed to the beam line through this tunnel, we will use it for the control room connections as well.

\subsubsection{AC Power}

A new sub-station, which will be located south of ESB, will provide most of the power for the NLCTA. The AC distribution system includes $480 \mathrm{~V}$ step down transformers, pullboxes, disconnects, breaker panels and cabling.

\subsubsection{LCW and Fire Protection Sprinkler System}

The NLCTA components will be cooled with Low Conductivity Water (LCW). The primary source of this LCW is the $1801 \mathrm{LCW}$ high pressure system which feeds the Research Area, SLC Arcs, and the SPEAR/SSRL complex. The LCW is supplied at a pressure of $265 \mathrm{psi}$ and has a return pressure of approximately $40 \mathrm{psi}$.

The pressure requirements for cooling the magnets and power supplies vary from $30 \mathrm{psi}$ to $200 \mathrm{psi}$. The supply pressure to individual components will be reduced by pressure reducing valves (PRV's) where required. The main headers in the accelerator area will be 6 inches in diameter, reducing to 3 inches at each end. The design flow rate is $5 \mathrm{ft} / \mathrm{scc}$, which is fairly conservative, and will allow for future increases if necessary. A separate piping system will be installed for the corrector power supplies.

The accelerator sections will be cooled with temperature stabilized LCW, operating at $45^{\circ} \mathrm{C}$. The design temperature tolerance is $\pm 0.1^{\circ} \mathrm{C}$. Temperature will be regulated using a three-way valve, electric water heater, and digital controller. This system is a separate loop, with make-up from the $1801 \mathrm{LCW}$ return side piping (40 psi). 
The magnet and accelerator LCW distribution systems, outside and inside the shielded enclosure, respectively, are shown in Figures 16 and 17.

The fire protection system, which will be a wet pipe design, will be in accordance with NFPA 13. 


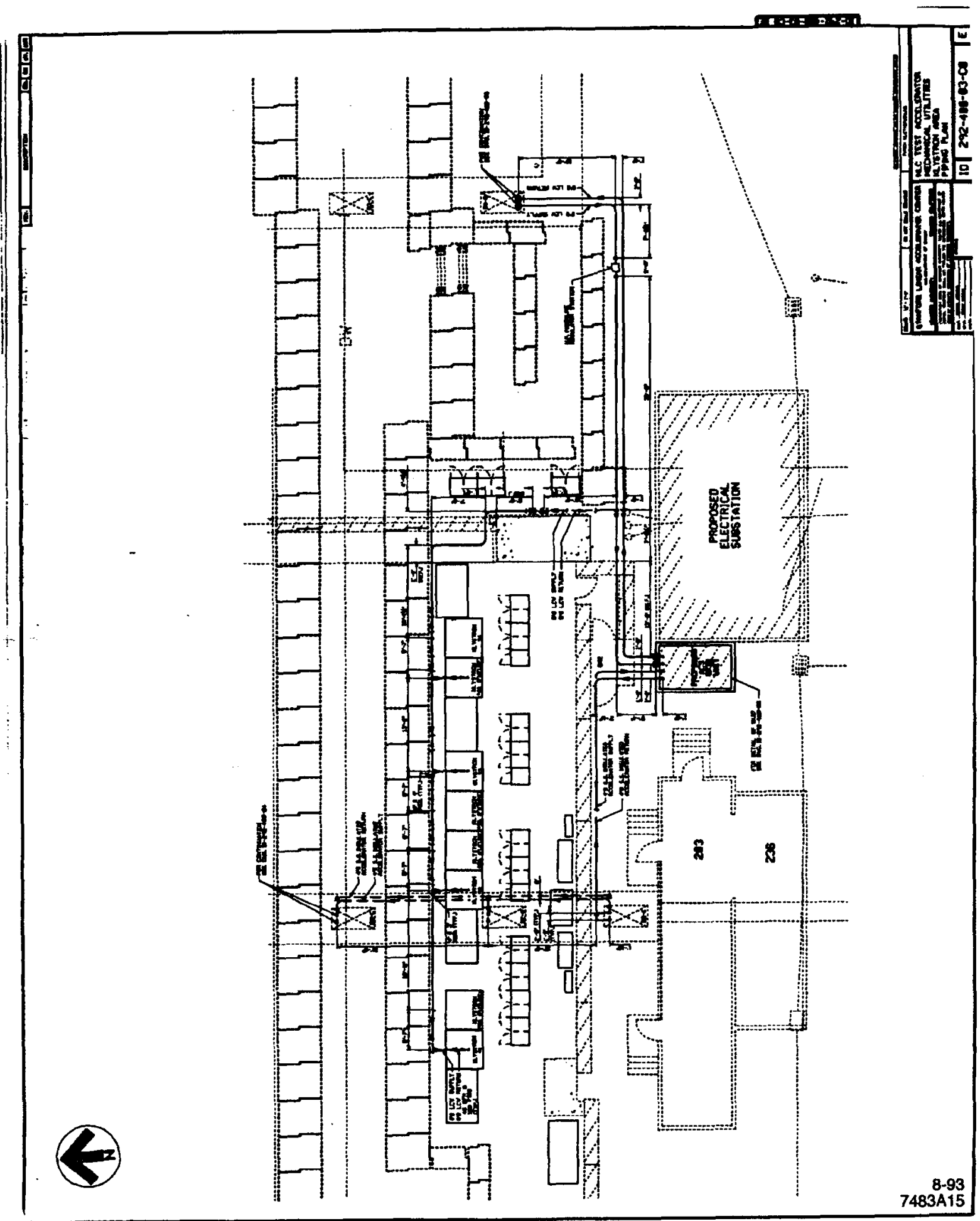

Figure 16. Magnet and accelerator LCW distribution system outside the shielded enclosure. 


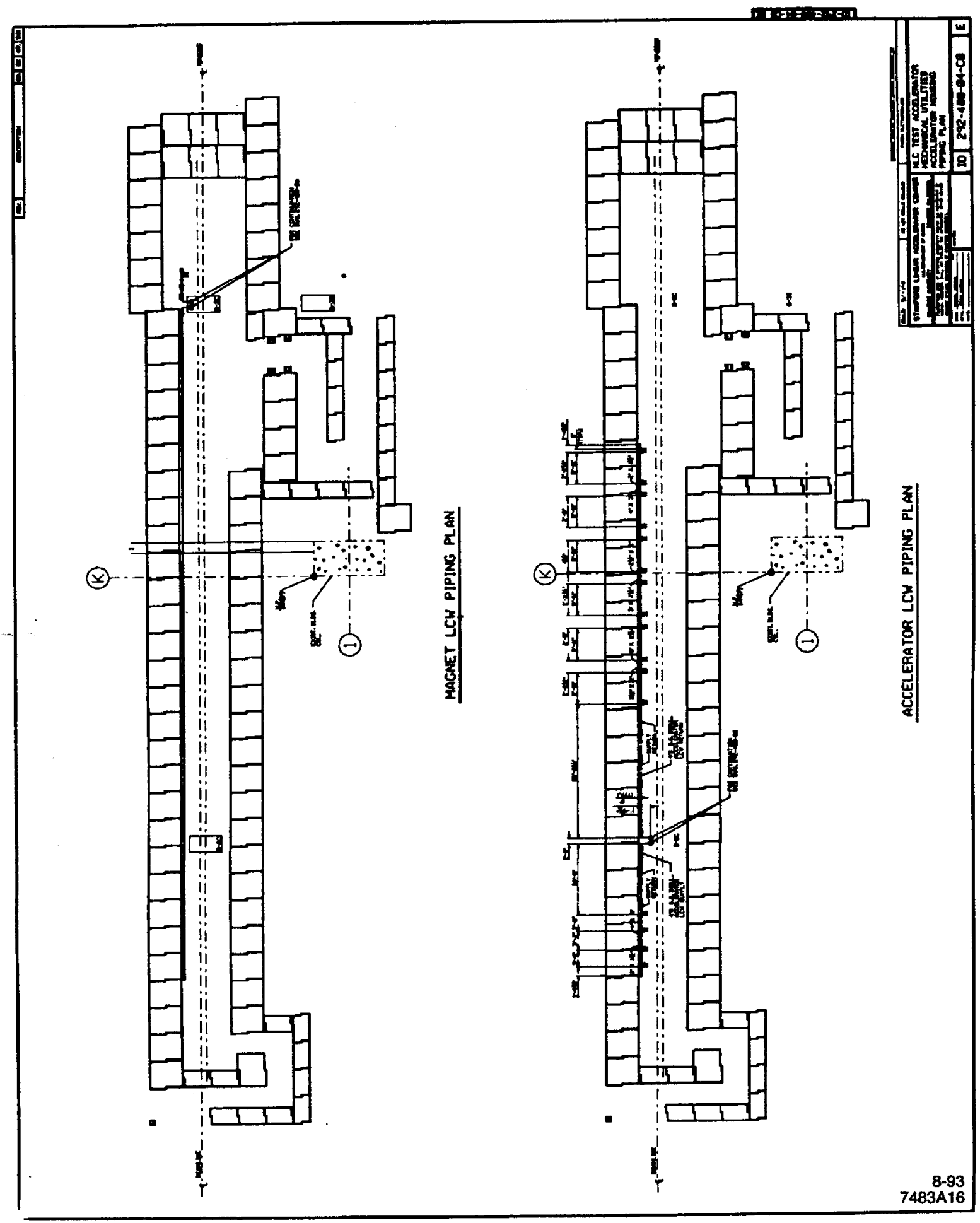

Figure 17. Magnet and accelerator LCW distribution system inside the shielded enclosure. 


\subsubsection{Cable Trays and Electronics Racks}

Cables will be run in 4-inch-deep ladder-type steel cable trays or other raceways from point of origin to termination. Barriers in the cable trays, or separate cable trays, will be used to separate different types of cables. In the utility tunnel under ESB, existing cable trays will be utilized and new sections will be added as needed. Inside ESB, cable trays will be placed above the I\&C racks, the DC power supplies and racks, the rf equipment racks, and along the beam line inside the shielded enclosure.

Equipment will be mounted in SLAC standard electronic racks. The racks will be outfitted in the shops with circuit breakers, plug mold strips, and internal rack power wiring. Wherever possible, the SLAC shops will load the electronic equipment into the racks.

The layout of the cable trays and electronics racks is show in Figures 18 and 19. 


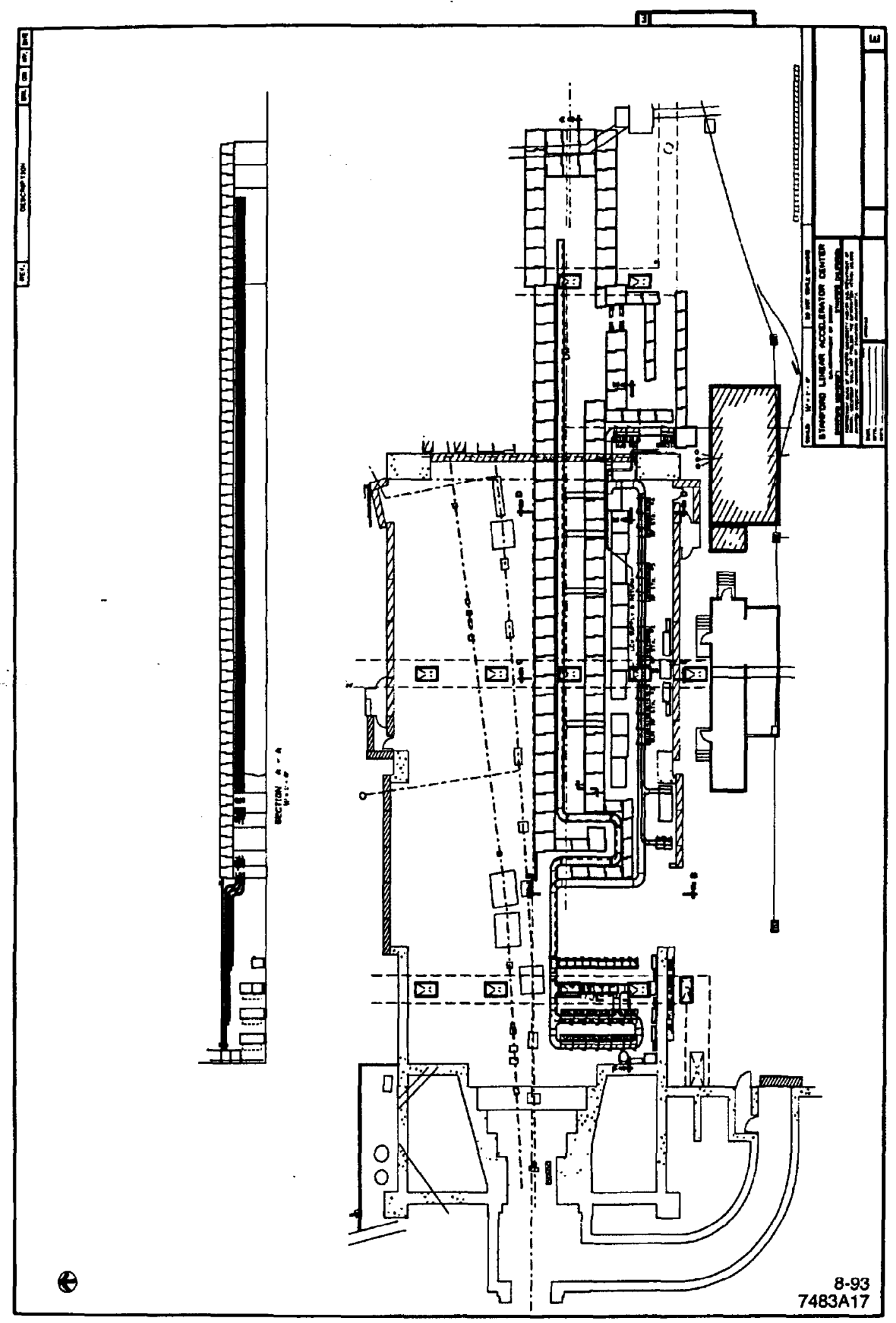

Figure 18. Layout of the cable trays and electronics racks. 


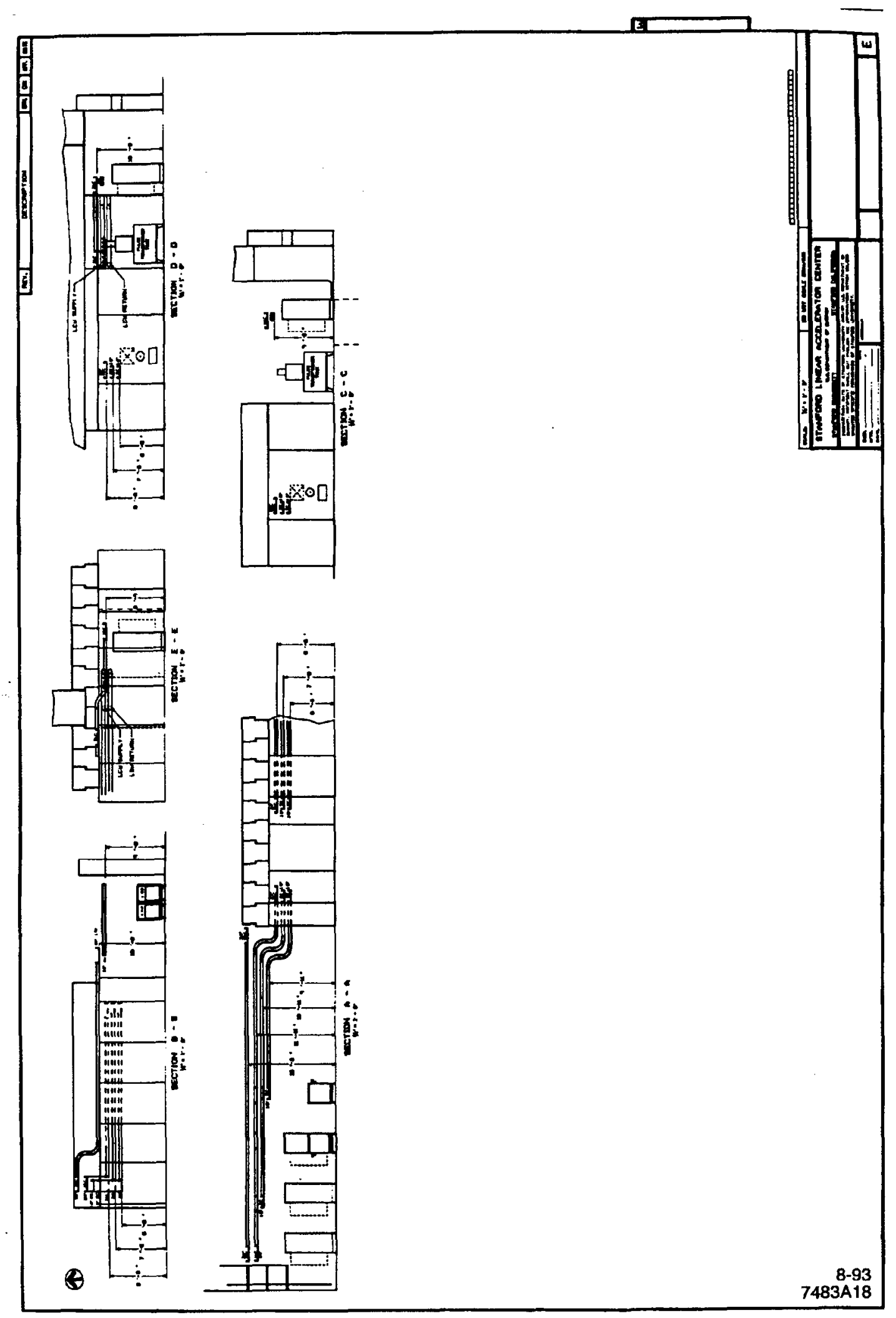

Figure 19. Elevation views of the cable trays and electronics racks. 


\subsection{SOURCE}

\subsubsection{Thermionic Gun}

For the electron source, we plan to fabricate a modern version of the thermionic gun presently used in the SLC. The gun will operate at $150 \mathrm{kV}$, produce a current of $2 \mathrm{~A}$, and have a variable pulse length of up to $150 \mathrm{~ns}$. Since very little documentation exists for the SLC gun, the fabrication of a gun will require the creation of a complete documentation package. ED\&I budget costs reflect this assumption. Piece costs are based on material and fabrication costs incurred during the recent construction of the Klystron Department ASTA gun and the SLC polarized gun. The high cost of the focus electrode arises from the expense associated with its polishing.

The gun's electronics costs are based upon estimates of commercially available equipment that are similar in design to that used for the SLC thermionic gun. As with the gun itself, the ED\&I costs are those required to upgrade and modernize both the electronics and controls. 


\subsection{VACUUM SYSTEM}

Beam line vacuum chambers will be made from 304 stainless steel and use knifeedge flanges and copper gaskets. Sputter ion pumps, distributed discretely along the beam line, will maintain nominal pressures of $10^{-9}$ Torr. A chamber bakeout at $200^{\circ} \mathrm{C}$ will take place in the laboratory prior to installation in the field. Pressure will be monitored with pairs of Pirani and cold-cathode gauge tubes.

The beam line vacuum system will be divided into seven regions: gun, injector, chicane, three linac regions (each consisting of two 1.8-In accelerator seclions), and spectrometer (including the straight-ahead line). Pneumatic, remotely operated, allmetal gate valves will isolate the regions. This will allow access to, or venting of, any one region, while adjacent regions remain under vacuum. Valves will also be interfaced to the pressure gauge controllers and will automatically close when pressures deviate outside of tolerance.

Each section will also have two all-metal valves providing an entrance and exit for dry nitrogen purges. Venting to dry nitrogen and pump down will also be done through these valves.

Each section will use one power supply and multiple ion pumps connected in parallel. Use of Pirani and cold-cathode gauge tube pairs, one pair per section, will allow pressure to be monitored from atmosphere to $10^{-11}$ Torr. Each gauge tube controller has two channels, reducing the number of controllers required by half. The gun's pressure will be monitored with a hot-filament gauge, allowing the possibility of in situ bakes in excess of $200^{\circ} \mathrm{C}$. 


\subsection{MAGNETS}

\subsubsection{Quadrupoles}

A total of 32 quadrupole magnets will be uscd in the chicane, linac, and spectrometer. Figure 20 illustrates the required quadrupole strengths for an isochronous chicane and a $100-\mathrm{MeV} / \mathrm{m}$ linac acceleration gradient. Also shown are the maximum quadrupole strengths that will achievable, given the choices of magnet designs and power supplies.

\section{QE-M Quadrupoles}

To meet the quadrupole requirements in the chicane, linac, and upstream end of the spectrometer, a modified version of the SLC Linac QE magnet design (denoted here by QE-M) will be used. One advantage of using the QE's is that SLAC owns enough laminations to make the number of magnets required. In addition, the magnets are fairly compact (16.5 cm long), and the SLC Linac style BPM, which is designed to fit into the 1.1 inch diameter bore of the magnet, can also be used in the NLCTA. Since the maximum operating strength of the standard QE $(\approx 100 \mathrm{kG})$ is much larger than needed, we decided to reduce the coil size to allow room for addition windings so the magnets can function as horizontal and vertical dipole correctors as well. Sextupole fields will be generated in addition to the dipole fields, but they will be small enough not to have an adverse effect on the beam transport.

The proper magnet designation for the quadrupoles commonly known as "QE's" is 1.1Q4.3. (The first number in this magnet designation format is the gap (for a dipole) or bore (for quadrupoles) in inches, the non-numeric character indicates the type of magnet ("D" for dipole, "Q" for quadrupole), and the second number is the length of the steel core in inches). We will use the laminations left over from the large-scale SLC QE production to make 27 (plus 1 spare) magnets with the modified coil package. The cost to produce the cores includes acquisition of a lamination de-burring machine and construction of a new fixture to stack the laminations.

The main coil will be wound using 0.1875-inch-square hollow copper conductor with a 0.125 -inch-diameter water cooling hole. Only the outer layer of the nominal two-layer package will be installed in each quadrant of the magnet: the remaining space will be filled with two solid-wire trim coils. The trim coils will be wound using \#12 square solid copper wire with heavy film insulation. The coils will be grouped into two sets of four, with one coil per quadrant in each set. The leads from the coils will be combined in series in each set to produce a horizontal dipole field when one set is powered, and a vertical dipole field when the other set is powered. In all, three power supplies will be connected to each magnet. These will provide independent control of the quadrupole, horizontal dipole, and vertical dipole field strength.

\section{QF Quadrupoles}

The requirements for the field strength and aperture (>50 $\mathrm{mm}$ diameter) for the last five quadrupoles in the beam line will be satisfied by the $3.15 \mathrm{Q} 12$ design (denoted 


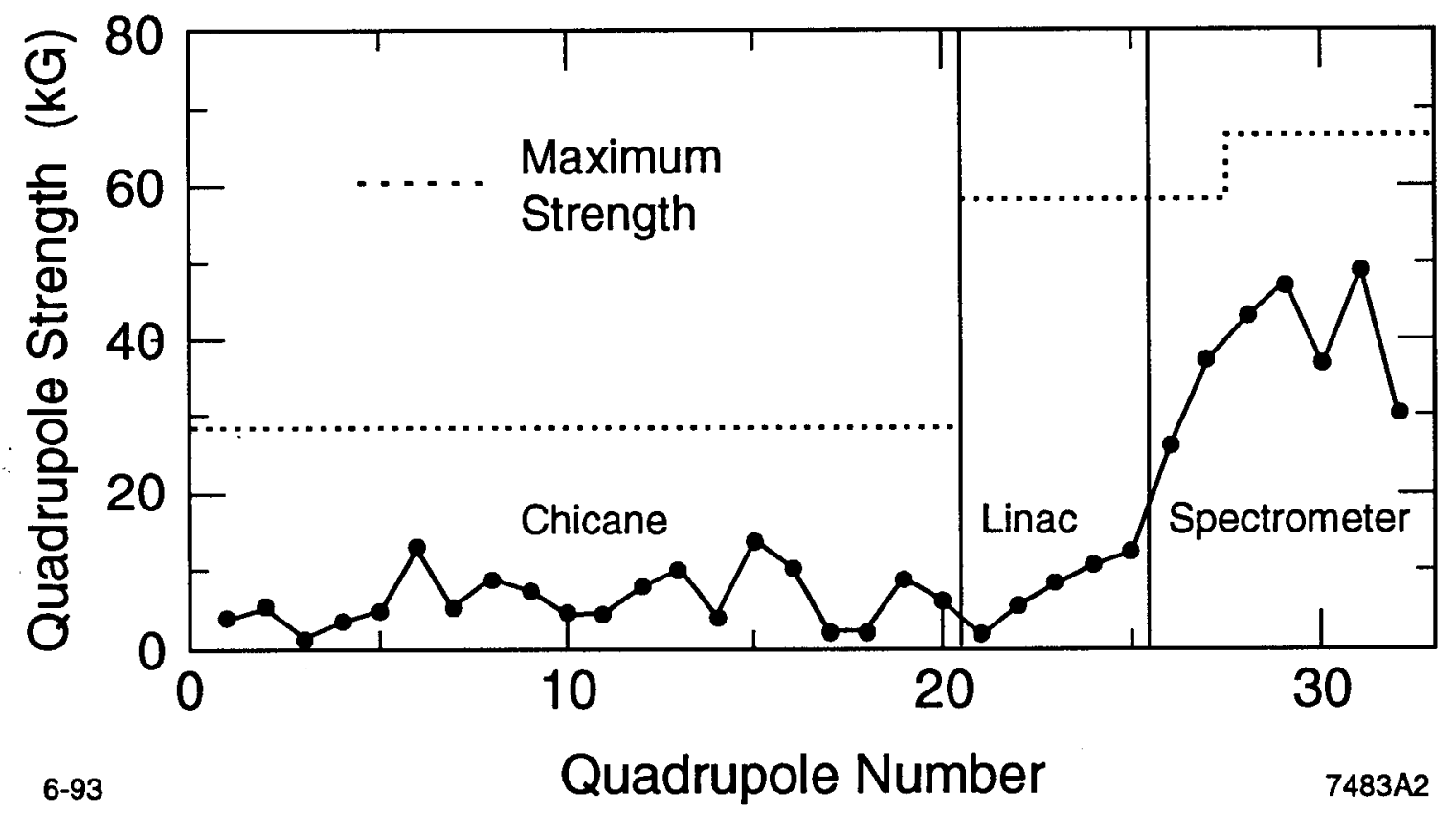

Figure 20. Required quad strengths for an isochronous chicane and a $100-\mathrm{MeV} / \mathrm{m}$ linac gradient. Also shown are the maximum quad strengths that will achievable, given the choices of magnet designs and power supplies. 
here by QF) that was used in SLC Final Focus. As with the QE's, SLAC has enough leftover laminations to make the number of magnets required. The laminations create a 1.625-inch bore. To enlarge this to 3.15 inches, the quadrant's cores will be stacked and welded, and then sent to an outside machine shop for grinding (this was how the Final Focus 3.15Q12's were made). The winding form and stacking fixture exist, and de-burring of the laminations will be done on the same machine as the QE magnets. The coils will be wound from 0.255 -inch-square hollow copper conductor with a 0.125 inch-round cooling hole.

\subsubsection{Dipoles}

\section{Chicane Bends}

The design of these bend magnets is basically the same as that of the 3D8.8MKII magnet made for the SLC Linac-to-Arc matching section. A $2 \%$ trim will be added by winding an extra layer of the same \#10 round wire that is used for the main coils. The desired integrated ficld strength of $0.57 \mathrm{kG}-\mathrm{m}$ can be achieved with a current of $7.8 \mathrm{~A}$.

The steel core will be machined out of solid low carbon steel. The winding form for the main coil exists and the trim coil will be wound on top of the main coil, eliminating the need for a trim winding form.

\section{Spectrometer Bend}

To bend the beam at its maximum energy of $1.2 \mathrm{GeV}$ into the $12^{\circ}$ spectrometer line requires an integrated field strength of $8.4 \mathrm{kG}-\mathrm{m}$. We will use an existing ' $\mathrm{H}$ shaped' magnet dipole for this purpose. The cost estimate for this magnet covers its refurbishing and mechanical support in the beam line.

\subsubsection{Dipole Correctors}

\section{Injector Short Correctors}

These will be air-core correctors based on the 2.97X/YC3.0 design used in the SLC injector. The coils are mounted on a hinged aluminum support which wraps around the vacuum pipe. They are made from \#13 square wire and can be operated at up to $5 \mathrm{~A}$.

\section{Injector Long Correctors}

These air-core correctors will be similar to those that have been wound in situ around the sub-harmonic-buncher in the SLC. They will use \#14 round copper wire, and can be operated at up to $4 \mathrm{~A}$.

\section{0-cm and 30-cm Spectrometer Correctors}

These correctors are denoted by their approximate length. In their construction, "bent" hot rolled steel plates will be used for their cores and \#10 round solid copper wire will be used for their coils. 
The "20-cm" correctors will be used to compensate the bcam steering caused by orbit offsets in the QF quadrupoles. They are 3.0D6.33's and have an integrated strength of $40 \mathrm{G}-\mathrm{m}$ at their maximum operating current of $8 \mathrm{~A}$. The "30-cm" corrector will be used to offset the average kick of the pulsed vertical kicker magnet during measurements of bunch energies. This corrector is a 1.625D9.24 and has an integrated strength of about $400 \mathrm{G}-\mathrm{m}$ at its maximum operating current of $8 \mathrm{~A}$.

\subsubsection{Solenoids}

\section{Injector Bucking Coil and Lens}

The initial focusing of the beams will be controlled by two solenoid magnets: a bucking coil (7.56SO1.68) and lens (3.1L3.0). The bucking coil is wound around the steel-encased lens to provide a means of controlling the fringe field inside the gun. These magnets will be very similar in design to the ones presently being used with the SLC polarized electron gun.

\section{Injector Large Solenoids}

Eighteen solenoid magnets with 42-cm inner diameters will focus the beam in the injector region. These solenoids plus three spares will be fabricated by an outside vendor using a design that the vendor has built before. The magnets will be operated at a current of $500 \mathrm{~A}$ which will produce a $0.8-\mathrm{kG}$ axial field.

\subsubsection{Kicker}

This iron-free magnet will be a standard SLC Damping Ring kicker. It will produce the required integrated field strength of $0.33 \mathrm{kG}-\mathrm{m}$ operating with a pulsed current of $2.8 \mathrm{kA}$. 


\subsection{Magnet Power Supplies}

The NLCTA will utilize power-supply designs that were developed during the SLC upgrade. The designs are well tested and will meet the NLCTA specifications of $10^{-4}$ or better regulation of the large dipole magnets, and $10^{-3}$ or better regulation of all other magnets. Except for the chicane bends and large injector solenoids, each magnet will have a separate power supply. The chicane bends will be powered in series although they will have individual $2 \%$ trims. (The spectrometer magnet will also have a $2 \%$ trim.) Of the 18 large solenoids, six will be powered individually. The remaining 12 will be powered in three sets of four.

Table 10 lists the ratings of the power supplies that will be used, grouped by magnet type and current requirements.

Table 10. NLCTA Power Supply Requirements

\begin{tabular}{|c|c|c|c|}
\hline Magnet & $\begin{array}{c}\text { Number of } \\
\text { Supplies }\end{array}$ & $\begin{array}{c}\text { Max. Required } \\
\text { Current (A) }\end{array}$ & $\begin{array}{c}\text { Power Supply } \\
\text { Rating }\end{array}$ \\
\hline - QE-M & 20 & 100 & $20 \mathrm{~V} / 125 \mathrm{~A}$ \\
\hline QE-M & 7 & 225 & $20 \mathrm{~V} / 250 \mathrm{~A}$ \\
\hline QF & 5 & 390 & $40 \mathrm{~V} / 400 \mathrm{~A}$ \\
\hline Chicane Bends & 1 & 8 & $20 \mathrm{~V} / 50 \mathrm{~A}$ \\
\hline Spectrometer Bend & 1 & 400 & $40 \mathrm{~V} / 400 \mathrm{~A}$ \\
\hline QE Correctors & 54 & 6 & $25 \mathrm{~V} / 6 \mathrm{~A}$ \\
\hline Dipole Correctors & 16 & 6 & $25 \mathrm{~V} / 6 \mathrm{~A}$ \\
\hline Dipole Correctors & 8 & 8 & $35 \mathrm{~V} / 12 \mathrm{~A}$ \\
\hline Bucking Coil and Lens & 2 & 6 & $25 \mathrm{~V} 6 \mathrm{~A}$ \\
\hline Solenoid Set 1 & 3 & 500 & $100 \mathrm{~V} / 600 \mathrm{~A}$ \\
\hline Solenoid Set 2 & 6 & 500 & $30 \mathrm{~V} / 800 \mathrm{~A}$ \\
\hline Kicker & 1 & 2800 & $1000 \mathrm{~V} / 5 \mathrm{~A}$ \\
\hline
\end{tabular}




\subsection{BeAM Devices}

NLCTA operations and experiments will require instrumentation for measuring the position, energy, transverse size, length, spacing, and intensity of bunches. We plan to measure each of these quantities averaged over the bunch train, as well as their variation within the bunch train. Since the bunch parameters are not expected to vary significantly on the sub-nanosecond time scale, the instrumentation will not need to resolve the properties of the individual bunches in the 11.424-GHz bunch train. Achieving even a few nanoseconds of resolution, however, will still require much development. In some cases, we will try more than one approach and see what works best.

The layout of the instrumentation planned for the NLCTA is illustrated in Figures 21-24. The analysis system contains 37 beam position monitors, eight profile monitors, four wire scanners, six toroid intensity monitors, six wall current monitors, one bunch length monitor, one bunch spacing monitor, and one Faraday Cup. The Faraday Cup, which will be located at the downstream end of the chicane, will also serve as a beam dump. In addition, there will be a non-instrumented beam dump at the end of each of the two spectrometer lines. Beam collimation will be achieved using two sieve eollimators, one adjustable four-jaw collimator, and seven fixed collimators. Most of the beam devices will be modeled after existing devices in the SLC.

\subsubsection{Bunch Position}

The transverse position of the bunches will be measured using components of the beam-position monitor (BPM) systems that were designed for the SLC and the Final Focus Test Beam. Strip-line BPMs will be located in the bores of all quadrupole magnets and at a few other locations. Each of these consist of four, 5-inch-long, 50 Ohm striplines, positioned 90 degrees apart in azimuth about the beam axis. The strip-line signals will be sent to electronics that track, hold on the peak value, and then digitize the signals. The BPM electronics will be gated to trigger on the leading peak of the bipolar strip-line signal, and will measure the average position of roughly the first $5 \mathrm{~ns}$ of the bunch train with a resolution of about $10 \mu \mathrm{m}$. If a stable trigger can be achieved on the trailing (inverted) peak of the strip-line signal, as well, then head-to-tail comparisons can be made.

Because standard SLC Linac strip lines will be used, a complete print package exists for these and very little ED\&I will be required. Tooling for the manufacture of these BPMs no longer exists and must be re-fabricated. Because new tooling will be used, a prototype will be made prior to production.

The signal processing electronics will consist of a head amplifier/filter in an rf chassis, followed by a track-and-hold module implemented with CAMAC control. CAMAC controlled pulsers will be used to measure the channel gains. A likely modification to this system will be the use commercially available high-speed waveform samplers (that are being considered for the rf monitoring system) to record the entire waveform from cach of the four strips on some of the BPMs. These data could 


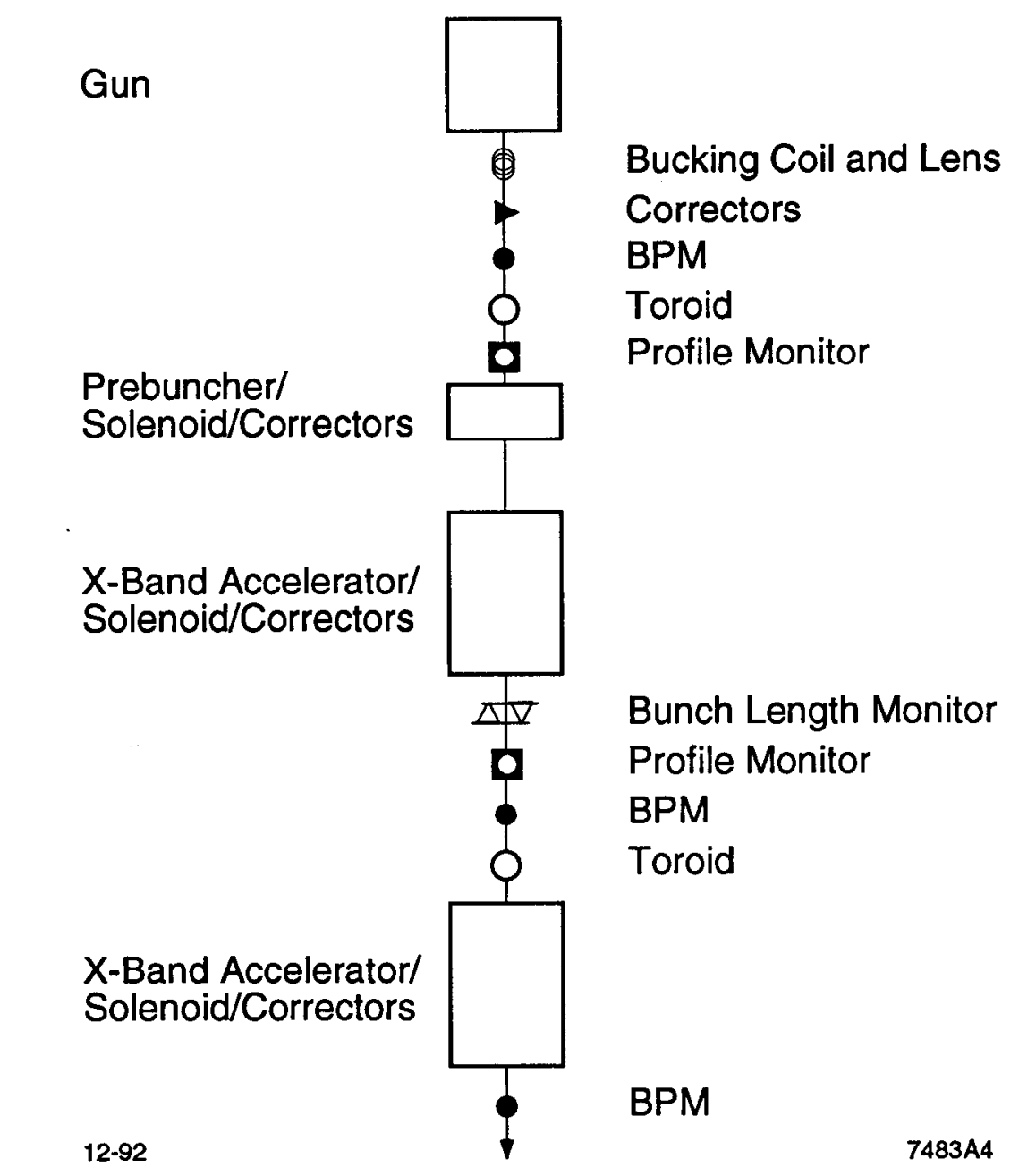

Figure 21. NLCTA injector beam instrumentation. 


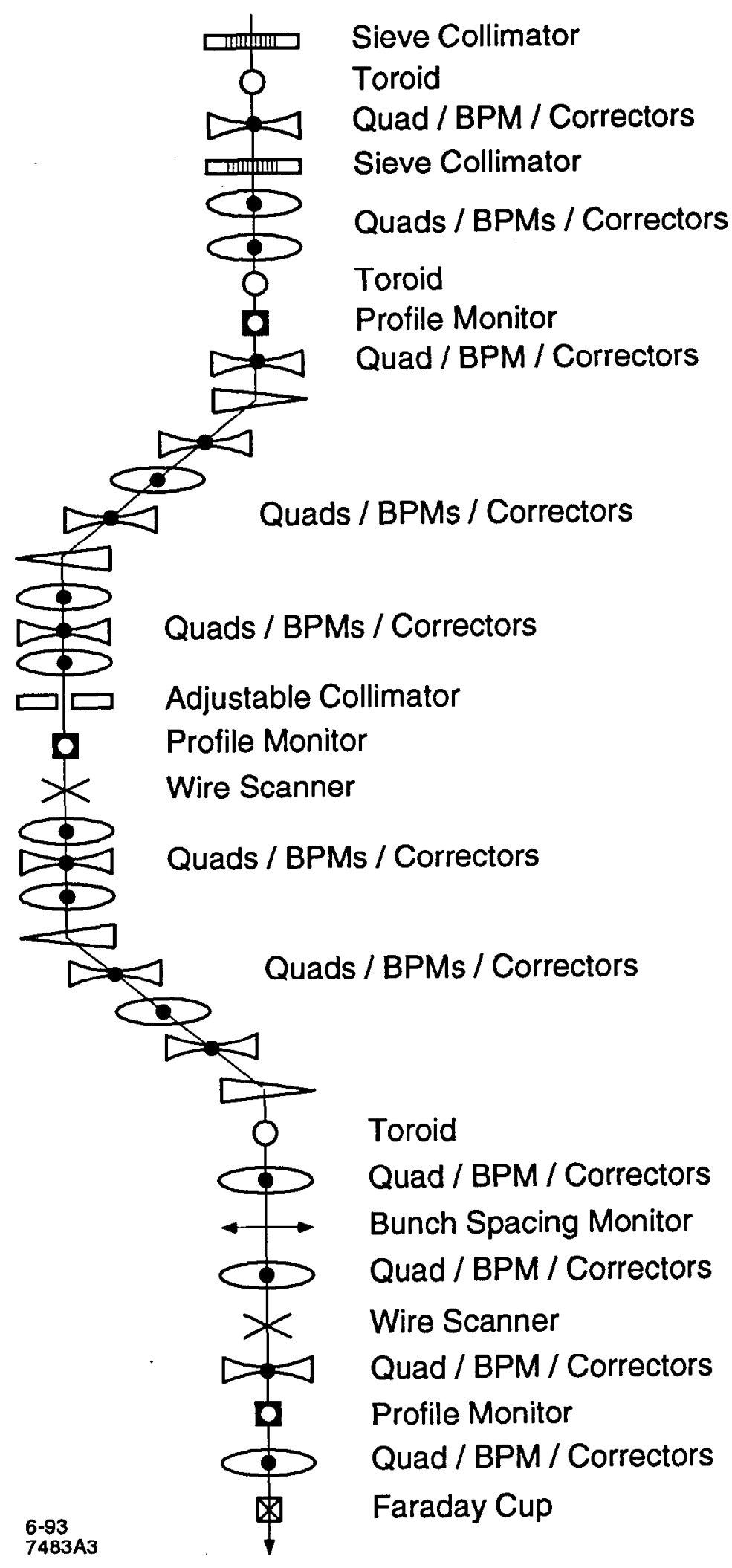

Figure 22. NLCTA chicane beam instrumentation. 
X-Band Accelerator

X-Band Accelerator

X-Band Accelerator

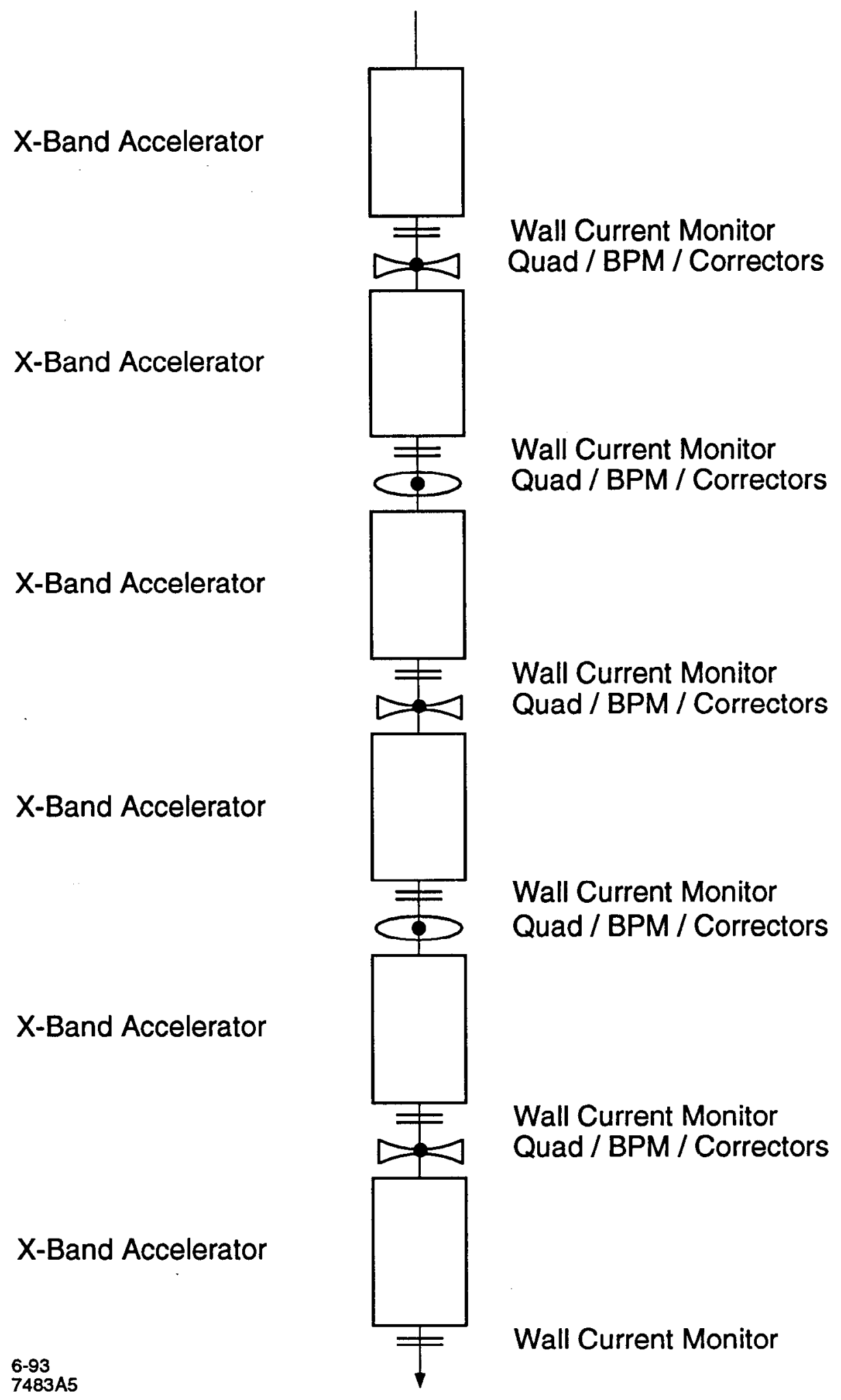

Figure 23. NLCTA linac beam instrumentation. 


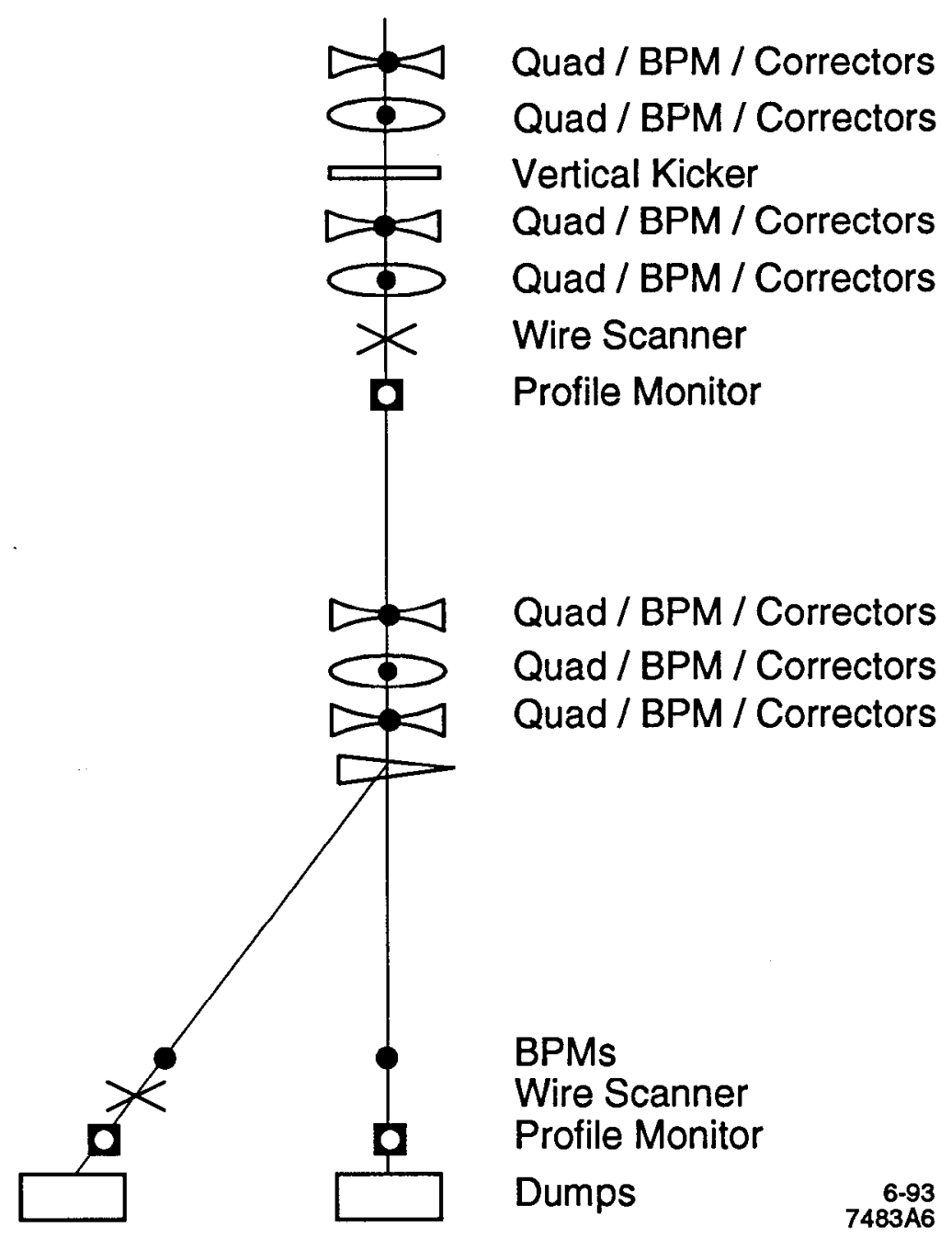

Figure 24. NLCTA spectromeler beam instrumentation. 
be analyzed to determine the variations of the transverse positions along the bunch train. Preliminary studies show that a resolution of about $10 \mu \mathrm{m}$ can be achieved for the Fourier components of the variations in transverse position with periods greater than a few tens of nanoseconds.

\subsubsection{Bunch Profile}

Transverse beam size and position will be measured by profile monitors and wire scanners. They will be installed at special locations along the beam line in order to optimally measure different properties of the beam. For energy measurements, they will be positioned at locations of high-dispersion in the chicane and spectrometer. For emittance measurements downstream from the injector, they will be positioned approximately $90^{\circ}$ of betatron phase downstream from quadrupole magnets. To measure emittance, the strength of a quadrupole will be varied, and the correlation between beam size and quadrupole strength will be used to extract the emittance and the match condition of the beam to the lattice.

\section{Profile Monitors}

During the profile monitor measurements, a video camera that is focused on an insertable-phosphor screen (aluminum coated with Gd2O2S:Tb) records the image of the bunch train as it passes through the screen. This information will be used primarily to measure the average position and size of the entire bunch train. With the magnetic spectrometer, however, we will have the ability to resolve transverse position and size variations within the bunch train by using a vertical kicker magnet that is ramped-up while the bunch train passes, thus separating the vertical positions of the bunches at the downstream profile monitors. The ramp period of the kicker will be adjustable in the range of 30 to $100 \mathrm{~ns}$ as a means varying the time-resolution. For the shortest ramp period, changes in beam position and size on the 1-ns timescale will be measurable with about a $100-\mu \mathrm{m}$ resolution. This corresponds to about a $0.02 \%$ resolution for energy and energy spread at the profile monitor at the end of the spectrometer line where $\eta_{x}=0.5 \mathrm{~m}$. Other profile monitors will be located at the intermediate focal point of the spectrometer and in the straight-ahead line, enabling precise measurements of the horizontal positions and sizes of the undispersed bunches.

Five of the eight profile monitors will be identical to those used in the SLC Linac and Damping Rings. The remaining three will differ only in aperture. The profile monitor control system will use an existing SLC design. A single chassis provides monitoring and control for two profile monitors, so four chassis will be used. These will be linked together for video transmission, allowing only one profile monitor screen to be viewed at a time. Each chassis will be controlled by standard SL $\Lambda$ C designed CAMAC modules.

\section{Wire Scanners}

Wire scanners will be installed in close proximity to half of the profile monitors. A wire scanner measures transverse bunch size by moving a thin wire at a controlled 
speed across the beam over a period of many pulses. Secondary emission produccd by the beam intercepting the wire on each pulse is detected by an off-axis photomultiplier tube. The correlation between the photo-multiplier signal and the wire position is used to reconstruct the transverse profile of the bunch train. Compared to the video profile monitor, the wire scanner is more convenient to operate and has much faster set-up time, much larger dynamic range, and better linearity. However, the wire scanners only measure projections of the bunch shape, and the data are taken over many beam pulses so the measurement is sensitive to pulse-to-pulse jitter of the bunch positions. For these reasons, we intend to use the wire scanners to do routine measurements of the average bunch size, and to provide a cross check of the video profile monitors. We are also looking into the possibility of high-speed waveform sampling for the photo-multiplier tube signals, or for the secondary emission signals from the wires themselves, in order to be able to resolve in time the sizes and positions of the bunches averaged over many machine pulses. Another device that would provide the same type of information is the streak camera (discussed below) that is being considered for bunch-length measurements in the chicane.

Thrce of the four wire scanners that will be installed will have standard SLC Linac designs. They will have a $30 \mathrm{~mm}$ scan range and contain 50-micron thick tungsten wires: The fourth scanner, which will be located at the end the $12^{\circ}$ spectrometer line, will have a $5 \mathrm{~cm}$ scan range and contain 250-micron thick tungsten wires. Its design will be similar to that for the wire scanner located in the West Turn-Around region of the SLC Positron Line.

Control and monitoring electronics will likewise use existing SLC designs. The interface electronics for the wire scanners will consist of an industrial process controller chassis (Daytronics 10K1) with associated PC boards, LVDT sensors, and CAMAC Stepper Motor Controller (SMC) modules. Photo-multiplier tubes will be used to detect secondary emission from the wires. A LeCroy single channel CAMAC module will supply high voltage to each tube.

\subsubsection{Bunch Length and Spacing}

Bunch-length measurements will be important for the initial tuning of the injector. The phase and amplitude of the rf in the prebuncher will be adjusted, first using a bunch-length monitor. The bunch-length monitor will be a cavity, resonant at a harmonic of the accelerating frequency, that provides a signal proportional to the strength of the fields generated by the bunches. The rf phase adjustment will be refined using an absolute measure of the bunch length. One method to obtain this information exploits the high correlation between energy and longitudinal bunch position that can be achieved by adjusting the phase of the second $0.9-\mathrm{m}$-long accelerator section in the injector. With such correlation, the average transverse bunch size measured near the center of the chicane is proportional to the average bunch length, provided that the bunch-to-bunch energy differences do not overly smear out the distributions. To avoid this problem, and to make the initial tune-up of the injector casicr, a 1-ns-long bunch train will be used. 
Verifying the bunching in long bunch trains by this method may be difficult because of the energy spread from beam loading. Also, if NLC-like bunches are used, the charge per bunch will be large enough that single-bunch transverse wakefield effects may decrease the apparent correlation of energy and longitudinal position. For these reasons, more direct means of measuring the bunch length are being considered. One possibility is using a streak camera to measure the synchrotron light generated in the final bend magnet of the chicane. A similar system has been proposed for use in the SLC linac for measuring bunch lengths of roughly of the same size. Tests at the SLC will help us decide whether to use this system at the NLCTA.

The bunch spacing can be affected by the bunching process. Ideally, it should be at the 11.424-GHz wavelength; however, beam loading in the buncher section can modify the spacing, as can bunch-to-bunch energy differences if the bunches are compressed in the chicane. Although we believe such changes in the bunch spacing will be small, and hence will not generate a significant bunch-to-bunch energy difference when the bunches are accelerated, we still want to verify the bunch spacing empirically. To do this, a resonant cavity tuned to $11.424 \mathrm{GHz}$ will be installed in the beam line. The signal generated when the bunch train passes through it will be mixed with a reference signal from the rf system to produce a time-dependent phase-error signal. The variation of the error signal, which will be resolvable on the time-scale of a few nanoseconds, will provide a measure of the uniformity of the bunch spacing. Having established the uniformity, the phase of the bunches relative to the rf will be determined by varying each of the klystron phases and observing the bunch energies in either the chicane or the spectrometer.

\subsubsection{Bunch Intensity}

Average bunch intensity will be monitored using toroids located between regions of the beam line where beam losses are expected to occur. Monitoring and processing of the toroid signals will done by a SIC CAMAC module designed for this purpose. Wall current monitors, which will be primarily used for measuring dark currents in the linac, will also be used to monitor the beam current.

An insertable Faraday Cup will be used to measure the total charge of the beam at the end of the chicane. The primary function of this Faraday Cup, however, will be as a beam dump while the injector is being tuned. The Faraday Cup will be selected from several that were removed from the SLC as part of an upgrade for higher power operation. The control system for this device will be provided through one channel of a profile monitor control chassis.

Although the toroids and Faraday Cup have absolute calibrations that are good only to $1-5 \%$, their resolution for measuring intensity changes, for which we primarily will use them, is better than $1 \%$. For measuring the time-variation of bunch intensity, we will use some of the same instrumentation described above for resolving beam positions and sizes. For example, the waveform-sampled BPM should provide $1 \%$ resolution of intensity variations on the time scale of a few tens of nanoseconds. 


\subsubsection{Beam Collimation}

Two sieve collimators will be located near the upstream end of the chicane. They will be used to limit the transverse size of the beam, and to reduce, if necessary, the beam intensity. Each of the units will contain a variable-aperture collimator and a variable-transmission (sieve) collimator in the same vacuum chamber. The variable-aperture collimator will consist of an insertable slide on which a linear array of apertures of different diameters are cut. The variable-transmission collimator will be similar, except its slide will contain screens of different mesh dimensions. The positions of the two slides transverse to the beam axis will be independently controllable so that any combination of aperture and mesh size can be selected. A sieve collimator was designed for the SLC injector but was never built. This design will be modified to optimize the aperture and mesh sizes for the NLCTA parameters. A prototype will be built and used as one of the two collimators required.

Control of the sieve collimators will be accomplished through use of CAMAC stepper motor control modules, LVDT sensors, and a shared industrial process controller chassis. The interface electronics for these collimators will be combined with that for the wire scanners and adjustable collimator.

An adjustable collimator will be located near the center of the chicane and will be used to limit the energy spread of the beam. It will be built using the SLC Linac four-jaw collimator design. Although this design is intended for applications with high power beams, the additional material and construction costs associated with this capability will be more than offset by the cost saving from not having to design a new collimator. The interface electronics for this collimator will be combined with that for the wire scanners and sieve collimators.

Seven fixed collimators will be used to protect beam line elements from the beam losses expected in the first half of the chicane. They will be identical to those used in the FFTB project except for aperture size.

\subsubsection{Beam Dumps}

A beam dump will be located at the end of each spectrometer line. The horizontal width of the dumps will be large enough $(15 \mathrm{~cm})$ to intercept all electrons within the design energy acceptance of the lines $\left(20 \%\right.$ in the $12^{\circ}$ line and $50 \%$ in the straight-ahead line with the spectrometer magnet powered at $10 \%$ of nominal strength). Since the maximum beam power will only be $1.2 \mathrm{~kW}$, the dumps will be simple in design, consisting of large copper blocks with LCW cooling tubes attached to their surfaces. A special radioactive water system will not be required since the beam is heavily attenuated in the blocks before it reaches the LCW. 


\subsection{RF DRIVE SYSTEM}

Rf power for the NLCTA will be derived from the SLC $476-\mathrm{MHz}$ drive-line signal, brought into End Station B by coaxial line from the Main Control Center. The proposed system is outlined schematically in Figure 25. The incoming $476-\mathrm{MHz}$ CW signal will be processed through a train of preamps, level-set attenuators, and mixers to produce signals at 2856,8568 , and $11424 \mathrm{MHz}$, all in the power range 10-20 $\mathrm{dBm}$, which will be distributed to the four NLCTA high-power rf stations.

The 2856-MHz and 8568-MHz signals will be used for phase-reference purposes, and will be transmitted in a temperature-stabilized package approximately 60 feet long which runs parallel to the accelerator housing and above the racks containing the rf signal-processing equipment for the klystrons. The $2856-\mathrm{MHz}$ signal will be transmitted in a 0.5 -inch, low-loss, phase-stable coaxial cable. The $8568-\mathrm{MHz}$ signal will run in WR112 waveguide, which has about $\frac{1}{40}$ the attenuation of coax at that frequency.

The 11424-MHz signal will be amplitude-modulated into a pulse train (typically $1.5 \mu$ s at $180 \mathrm{~Hz}$ ), and then passed through a phase-shift-keying modulator, in which the phase of the rf is rapidly changed by $180^{\circ}$ at a selected time after the beginning of the pulse. This phase reversal, which takes place in a few nanoseconds, is required for the operation of the SLED-II pulse-compression system.

After further amplification, the 11424-MHz pulsed signal will be transmitted in WR90 waveguide along the temperature-stabilized package. Couplers in the coaxial and waveguide lines at each station will provide rf power for the drive chain to the klystron and for monitoring the phase and amplitude of selected signals from the accelerator system.

The drive chain to each klystron contains a further preamp, phase shifter, TWT pulsed amplifier, and level-set attenuator. 


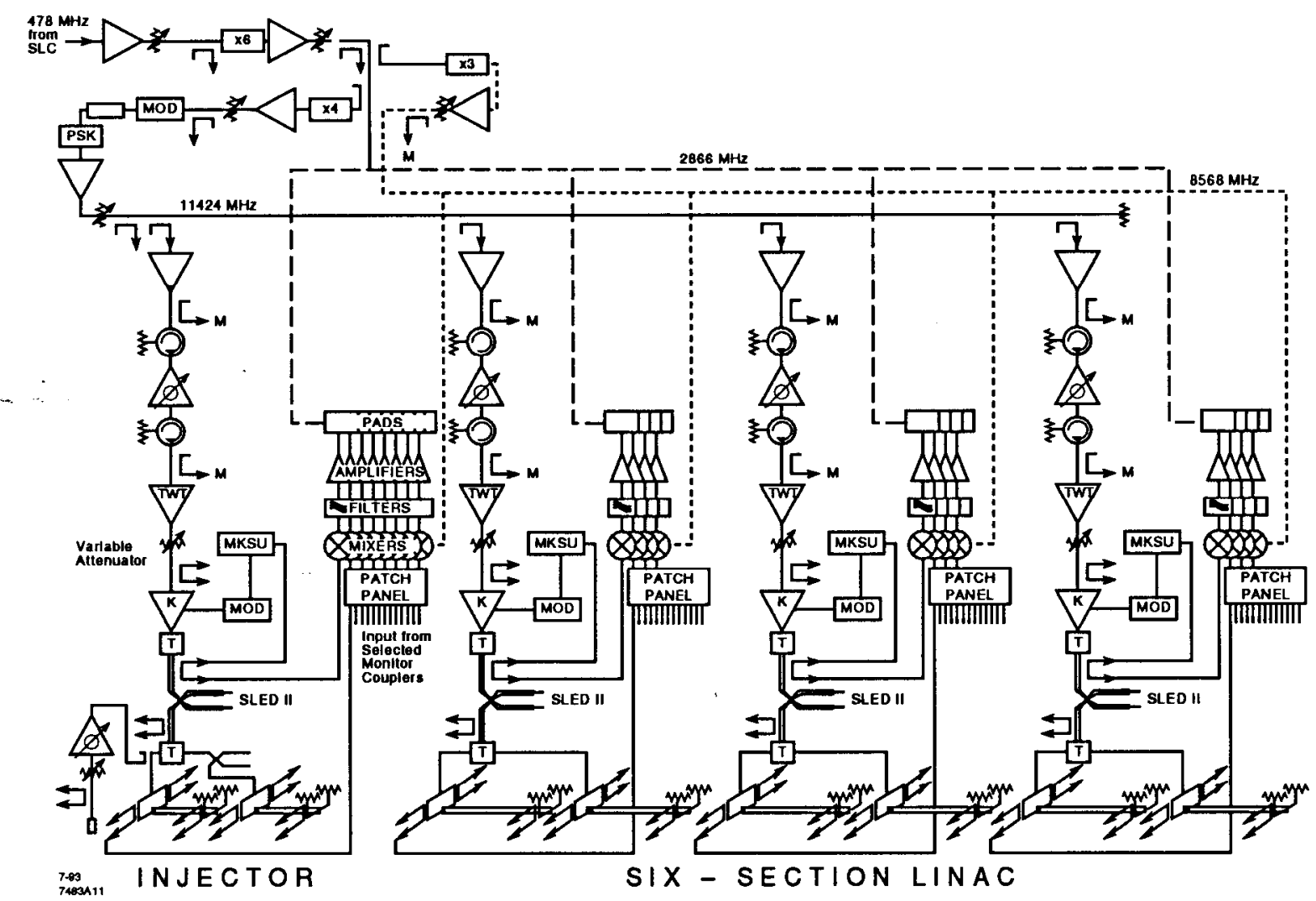

Figure 25. Rf drive- and control-system schematic. 


\subsection{RF Control and Measurement}

\subsubsection{RF Control}

Remotely-controlled phase shifters and attenuators in the low-power rf drive system, and in the high-power waveguide system, will be used to adjust the phases and amplitudes of the high-power rf feeds to the accelerator. The phase and amplitude of the low-power rf drive for each klystron will be adjustable. Power for the prebuncher cavity (about $1 \mathrm{~kW}$ ) will be coupled-off from the feed for the first injector accelerator section and passed through an adjustable phase shifter and attenuator. The rf phase of the second injector accelerator section relative to the first will be adjustable by a high-power phase shifter. The phase-length of the high-power SLED-II delay lines will be controlled by adjusting (by closed-loop feedback) the position of a shorting plunger at the end of each waveguide delay line.

Feedback loops for rf control will be implemented within the framework of the SLC control system hardware and software. One feedback loop will monitor and stabilize klystron output power by adjusting the driver phase shifter and attenuator. 'The comparable feedback loop in the SLC rf system is under the control of a local processor which also protects the klystron against high-voltage breakdown, reflected power, and vacuum faults. New control and protection systems, specifically tailored to the X-band klystrons, will be developed for the NLCTA using the SLC-standard control modules and protocols.

Another feedback system will be developed to set and stabilize the SLED-II delay-line phase lengths. This feedback loop will have low bandwidth (less than $0.1 \mathrm{~Hz}$ ), appropriate to the thermal expansions and contractions of the 120-foot-long waveguides that must be compensated. The information needed to set and stabilize the delay-line phase lengths can be derived from measurements of the phases and amplitudes of the SLED-II rf input and output pulses. The actuators of the feedback loops will be SLC-standard CAMAC-controlled stepper motors that move the delayline shorting plungers. Other rf feedback loops, if needed, can be implemented along the lines of this model.

\subsubsection{RF Phase and Amplitude Monitoring}

The monitor points (directional couplers) for phase and amplitude measurements are indicated by arrows in Figure 25. In order to reduce the number of channels of expensive electronics, only those rf signals required for feedback processes will be "hard-wired" into phase and amplitude detectors (PADs). Those rf signals needing only occasional monitoring will be routed to phase and amplitude detection circuits via patch panels when needed. Error waveforms generated by the PADs may be either viewed directly on an oscilloscope for evaluation and adjustment, or digitized and used for closed-loop control.

The NLCTA rf control system will employ the PAD system used in the SLC. The SLC PADs track, sample, hold, and digitize (12 bits) the amplitude and phase 
of a 2856- $\mathrm{MHz}$ signal once per pulse. (Repetitive waveforms are mapped-out by stepping the sample time during a series of pulses.) The SLC PADs are interfaced to the SLC control system via a CAMAC Parallel I/O Processor (PIOP). Two types of PAD systems, with bandwidths differing by a factor of two, are used at the SLC. The NLCTA will utilize the wider-bandwidth version (used in the SLC for measuring beam phase with respect to the rf) which has $30-\mathrm{ns}$ time resolution (15- $\mathrm{MHz}$ bandwidth). In order to use the SLC PADs in the NLCTA rf system, the 11424-MHz signals will be converted to $2856 \mathrm{MHz}$ by mixing with a stable $8568 \mathrm{-MHz}$ reference signal. The 30-nsec resolution of the SLC PADs will be adequate for monitoring the average phase and amplitude of the 1.5-microsecond-long klystron pulses and the 0.25 -microsecondlong SLED-II pulses in the NLCTA.

\subsubsection{Experimental RF Data Acquisition}

The experimental program of the NLCTA requires the ability to make rf phase and amplitude measurements with time resolution comparable to the NLC bunch spacing. It will therefore be necessary to detect $\mathrm{rf}$ phase and amplitude with approximately 1.4-ns time resolution, and to sample and digitize the detector video output at a rate of 0.5 to $1 \mathrm{GSa} / \mathrm{s}$ during the pulse. Since the goal for bunch-to-bunch energy stability is $0.1 \%$, one would like to measure phase to $0.1^{\circ}$ precision (out of $360^{\circ}$ ) and amplitude to $0.1 \%$ resolution along the pulse "flat top." This requires the development of a new high-speed, high-resolution phase and amplitude detector (a "Fast PAD") and data-acquisition system, with functional specifications as listed in Table 11. Fast PADs will be used for monitoring klystron, SLED-II, and accelerator rf pulses, and the X-band bunch-phase monitor.

Table 11. Preliminary Functional Specification for a "Fast PAD"

Signal frequency:

Time resolution:

Amplitude resolution:

Phase resolution:

Digital sampling rate:
$11.424 \mathrm{GHz}$

$1.4 \mathrm{~ns}$

$\leq 0.1 \%$ per $25 \%$ of full scale

$$
\leq 0.5 \% \text { per } 100 \% \text { of full scale }
$$

$0.1^{\circ} / 360^{\circ}$ relative to reference

$0.5-1 \mathrm{GSa} / \mathrm{s}$

Fast PADs are not commercially available and will require custom engineering development work. Video output waveforms from the Fast PADs can be sampled and digitized by gated high-speed digitizers and analyzed by local real-time processors which are networked to the main online control computer. Suitable digitizers and 
local processors are available commercially within the VXI instrumentation standard. ${ }^{*}$

The purpose of the local processing will be to reduce the digitized waveform data stream to statistical summary information for transmission to the main online computer, and to identify exceptional events for which more complete waveform data should be transmitted. The reduced data stream will be transmitted by Ethernet from the local processor to the main online computer where the summary data will be saved in "history buffers" and the complete waveforms of the exceptional events will be saved for further analysis.

Should it become desirable in the future to increase the sophistication of $\mathrm{rf}$ control in the NLCTA, feedback loops may be designed which will utilize the of waveform information acquired by the Fast PAD system.

* A Tektronix subsidiary (Analytek, Ltd., Sunnyvale, California) offers the 2004S VME fourchannel sampling module with $300-\mathrm{MHz}$ analog bandwidth, $0.5-\mathrm{GSa} / \mathrm{s}$ sampling rate, 12-bit ADC, and 2048-point memory per channel. Hewlett-Packard offers the E1428A VXI twochannel digitizing oscilloscope with $250-\mathrm{MHz}$ (3-dB) analog bandwidth, up to 1-GSa/s (simultaneous) sampling rate, 8-bit ADC, and 8000-point memory per channel. 


\subsection{Pulse Modulators}

The NLCTA 50-MW klystrons could in principle be driven by the same SLACtype modulators now in use to power the X-band klystron test stands in the Klystron Test Laboratory. Parameters for the SLAC-type modulator, using a 20:1 turns ratio pulse transformer but having a longer pulse flat-top (1.5 $\mu \mathrm{s} v s .0 .9 \mu \mathrm{s})$, are shown in Table 12. However, this high pulse-transformer turns ratio leads to a large leakage inductance and hence to long pulse rise- and fall-times, implying a low modulator efficiency. The energy contained in the flat-top portion of the pulse applied to the klystron is only $58 \%$ of the energy stored in the capacitors of the pulse-forming network. This poor energy-transfer efficiency implies an excessive wall-plug power for a full-scale NLC.

Table 12. NLCTA Modulator Parameters

SLAC-Type: Standard SLAC modulator with 20:1 Pulse Transformer. Proposed: 1.5-times multiplying Blumlein PFN.

\begin{tabular}{|l|c|c|}
\hline & SLAC-type & Proposed \\
\hline Charging voltage $(\mathrm{kV})$ & 50 & 70 \\
Pulse voltage $(\mathrm{kV})$ & 480 & 600 \\
Pulse $\pm 1 \%$ flat-top $(\mu \mathrm{s})$ & 1.5 & 1.5 \\
Pulse rise-time to $99 \%(\mu \mathrm{s})$ & 1.0 & 0.4 \\
Pulse energy width $(\mu \mathrm{s})$ & 2.5 & 1.9 \\
Energy-transfer efficiency & 0.58 & 0.77 \\
Net modulator efficiency & 0.54 & 0.73 \\
\hline
\end{tabular}

A new modulator design has been proposed ${ }^{33}$ that uses a multiplying Blumlein (or Darlington) pulse-forming network, which allows a reduction in the turns ratio of the pulse transformer to 6:1 for the same output pulse voltage. This leads to decreased rise-time and better energy-transfer efficiency, as indicated in Table 12. The output pulse shape for the proposed modulator design is compared with the present SLAC modulator pulse in Figure 26. The improved rise-time and a substantial gain in efficiency are evident from the figure. Conversion of a standard modulator in the Klystron Test Laboratory to the new design for testing is planned during FY93.

The conversion will involve rebuilding the modulator with a new Blumlein pulseforming network and a new pulse transformer. The present thyratron and charging supply will be retained. While this design provides sufficient pulse voltage and current for the 50-MW X-band klystron, a pulse voltage on the order of $600 \mathrm{kV}$ will be 


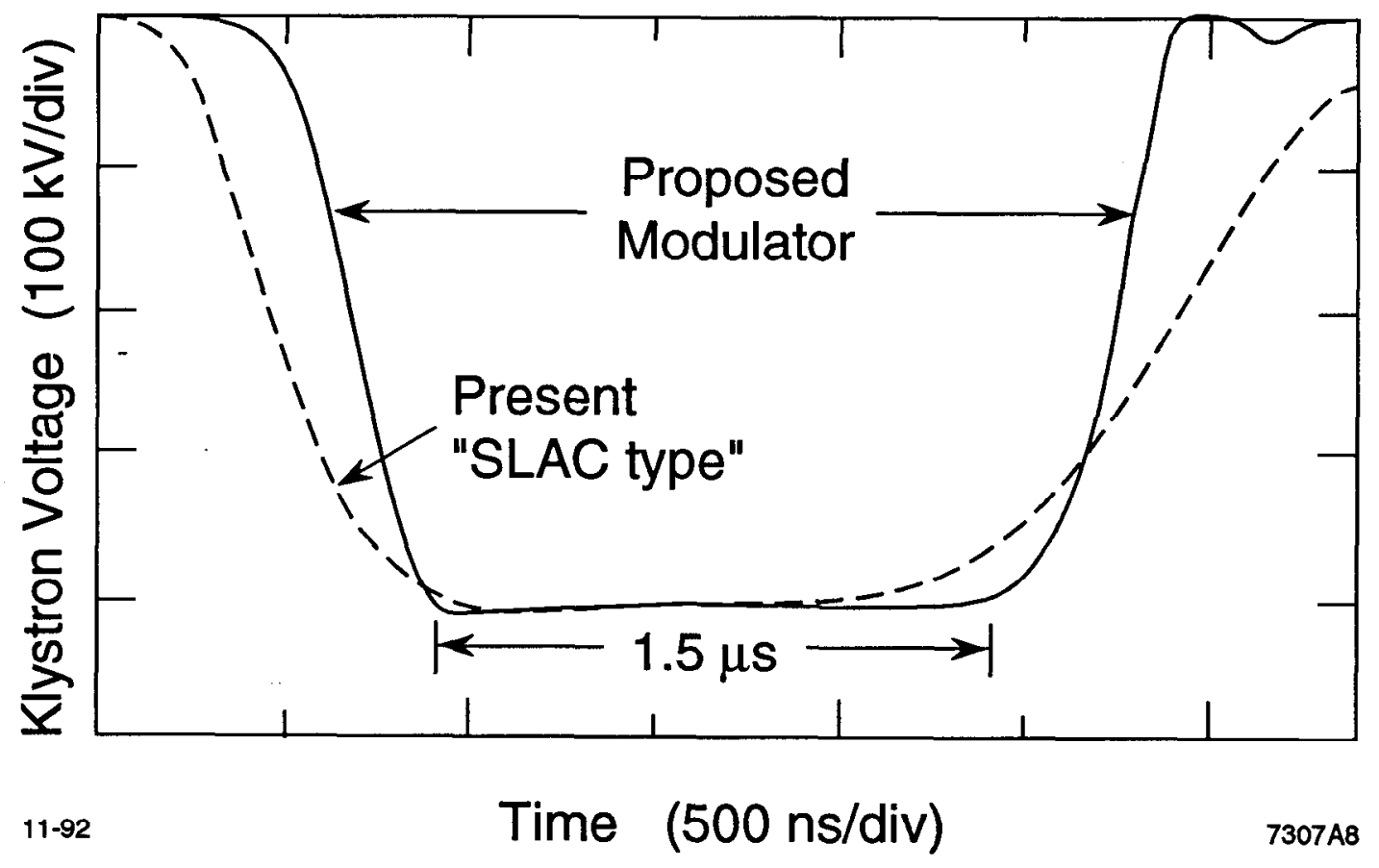

Figure 26. Comparison of a SLAC-type modulator (scope trace) with a simulation of the proposed modulator. 
needed to produce power at the 100-MW level necessary for a $100-\mathrm{MV} / \mathrm{m}$ accelerating gradient. Table 12 lists the parameters for a proposed modulator using a thyratron and dc charging supply with a higher voltage capability, which increases the voltage on the pulse-forming network to $70 \mathrm{kV}$.

Note that in Table 12 the net modulator efficiency is lower than the energytransfer efficiency referred to above. This difference is due to losses in the input transformers, dc power supply and the resonant charging circuit. This "front end" efficiency is taken to be $93 \%$ for the present modulator design and slightly higher for the proposed design. 


\subsection{KLYSTRONS}

\subsubsection{0-MW Klystrons}

The NLCTA will be powered by four 11.424-GHz klystrons with 50-MW peak power capability at $1.5-\mu$ s pulse length. These tubes will operate at a microperveance of $1.2(350 \mathrm{~A}$ at $440 \mathrm{kV}$ ). The $50-\mathrm{MW}$ goal requires only $33 \%$ beam-to-rf conversion efficiency. However, much greater efficiency and power are expected to be achieved. The basic 50-MW klystron design parameters are summarized in Table 13 . The 50MW goal considerably eases the design challenges relative to the 100-MW XC series, described in Section 2.3, where the microperveance was 1.9 (550 A at $440 \mathrm{kV}$ ) and the $100-\mathrm{MW}$ goal required $43 \%$ efficiency.

Table 13. 50-MW Klystron Design Parameters

\begin{tabular}{|l|c|}
\hline Operating frequency & $11.424 \mathrm{GHz}$ \\
Peak output power & $50 \mathrm{MW}$ \\
Rf pulse width & $1.5 \mu \mathrm{s}$ \\
Pulse repetition rate & $180 \mathrm{pps}$ \\
Rf pulse rise-time & $\leq 10 \mathrm{~ns}$ \\
Beam voltage & $440 \mathrm{kV}$ \\
Beam current & $350 \mathrm{~A}$ \\
Beam-to-rf efficiency & 0.45 \\
Saturation gain & $55 \mathrm{~dB}$ \\
Focusing field & $5 \mathrm{kG}$ \\
\hline
\end{tabular}

The NLCTA klystrons will use a single $\mathrm{TE}_{01}$-mode output window, one-halfwavelength thick, preceded by a "flower-petal" transition from the rectangular waveguide of the klystron output to the circular $\mathrm{TE}_{01}$-mode waveguide in which the window is mounted. Designs for both the mode transducer and the window have been completed.

The NLCTA klystron output circuits will be either standing-wave or travelingwave structures of the disk-loaded waveguide type. Designs for both standing-wave and traveling-wave output circuits exist for which the computer modeling predicts outputs greater than $70 \mathrm{MW}$ at $450 \mathrm{kV} .{ }^{*}$ The standing-wave version is in cold-test and will go into the first 50-MW klystron prototype, designated "XL1." This tube will be tested, with a $\mathrm{TE}_{01}$-mode output window, in September 1993.

* Simulations of the design have been performed using a two-dimensional particle-in-cell code (CONDOR) assisted by three-dimensional rf-field modeling (by MAFIA). 
It is expected that the second prototype tube of the XL series will incorporate a traveling-wave output circuit. The decision on the final design for NLCTA production will be based on the performance of the first two XL-series klystrons. At least four XL tubes then will be produced to provide the NLCTA complement of four tubes, and to provide spares.

\subsubsection{0-MW Klystrons for a Future Energy Upgrade}

R\&D toward the full-power NLC klystron will resume after the NLCTA requirements have been met. The 100-MW design will also be bascd on a lowcr-pcrveance beam and will require a $550-\mathrm{kV}$ cathode voltage. A modulator is under construction for this purpose. The output circuit will be an extension of the design used for the 50-MW tubes. The $\mathrm{TE}_{01}$-mode windows, and the "flower-petal" transitions to them, will be identical to those used in the XL series. The principal differences between the XL klystrons and the final 100-MW tubes will be in the gun and collector. Both will be larger to accommodate the higher voltage and beam power. 


\subsection{RF Pulse Compression and Power Transmission}

To obtain high peak power with a 0.25 -microsecond flat-top at $11.424 \mathrm{GHz}$, a new method of pulse compression, SLED-II, ${ }^{12-15}$ will be used. Four SLED-II rf pulse compressors, one per klystron, will compress the klystron output pulses from $1.5 \mathrm{mi}$ croseconds to 0.25 microseconds. The intrinsic efficiency of the SLED-II compression method, for this compression factor, is $75 \%$. The overall rf system design, discussed in Section 2.2, requires that the net efficiency of each pulse compressor (intrinsic $\times$ component efficiency) be at least $67 \%$. Therefore, the total losses in the microwave components of each SLED-II must not exceed 11\%. Development work currently is underway to devclop the necessary low-loss components. For some particularly critical components, both primary and alternate approaches are being developed simultaneously to ensure the availability of an acceptable design on schedule.

An experimental SLED-II pulse compressor is in use with an experimental Xband klystron, providing high power for tests of X-band accelerator structures in the Accelerator Structure Test Area (ASTA) ${ }^{34}$ in the Klystron Test Lab at SLAC. The SLED-II hardware-development program is based on continuous incremental modifications to the experimental prototype, as improved components are developed. The final SLED-II prototype for the NLCTA should be completed in 1994. The protolype will be used for high-power conditioning and acceptance testing of the production versions of NLCTA accelerator sections.

SLED-II is not our first experiment with high-power X-band pulse compression. Prior to the initiation of the SLED-II experimental program, a high-power Binary Pulse Compressor (BPC), which used oversized waveguide components similar to those required for SLED-II systems, was used successfully to provide power to ASTA for the high-gradient accelerator field-emission studies described in Section 2.4.2. In the BPC, rf pulses were compressed by a factor of eight, with a factor of five increase in peak power. ${ }^{35}$ The highest peak output power achieved in binary pulse compression was $160 \mathrm{MW}$ in a 70 -ns pulse. This peak power was limited by the maximum available klystron power at the time of the test, and not by the pulse-compression system itself.

\subsubsection{Mode Transducers}

We have developed "flower-petal"-type mode transducers to make transitions from WR90 rectangular waveguide to the low-loss $\mathrm{TE}_{01}$ mode in circular waveguide. ${ }^{36}$ The transducers have been tested successfully in an X-band resonant ring at pulse energy up to $150 \mathrm{MW} \times 0.8 \mu \mathrm{s}$. Power losses in a typical transition (due to modeconversion, ohmic losses, and mismatches) total $0.7 \%$. Reflections due to impedance mismatch account for approximately $0.2 \%$ of this loss. Redesign for improved matching is expected to reduce the net power loss of a transition to $0.5 \%$.

\subsubsection{Circular Waveguide}

To minimize losses, rf power will be transmitted in the low-loss $\mathrm{TE}_{01}$ mode through circular oversized waveguide. Power will be transmitted from each klystron 
to its SLED-II pulse compressor, and from the pulse comprcssor to the accelerator, in three-inch-diameter waveguide, which has only $3 \%$ of the loss of standard X-band rectangular guide (WR90).

The SLED-II pulse compressors each require a pair of microwave "delay lines" with 0.25 -microsecond down-and-back transit time (in which the 0.25 -microsecondlong output pulse is formed). These delay lines also will be made of circular oversized waveguide, and will operate in the low-loss $\mathrm{TE}_{01}$ mode. Pulse-compression expcriments (Binary and SLED-II) have been performed using 2.81-inch-diameter waveguide (WC281). A high-power SLED-II prototype is being constructed with 4.75-inch diameter delay lines (WC475). However, the final choice of waveguide diameter(s) for the NLCTA has not been made yet.

In general, the choice of diameter for both the transmission lines and the delay lines is based on the simultaneous goals of (1) avoiding cut-off diameters where unwanted modes can become trapped and resonate between discontinuities (such as imperfect flange joints) and flower-petal apertures, and (2) reducing ohmic losses (which are inversely proportional to the cube of the waveguide diameter). However, ohmic losses must be maintained at a level greater than losses due to mode conversion in order tõ supress undesired resonances of trapped higher-order modes. ${ }^{37}$

A $\mathrm{TE}_{01}$-mode-selective 50-dB directional coupler for monitoring power in circular waveguide has been designed and tested. ${ }^{38}$

\subsubsection{Mode Suppressors}

Unwanted modes that reflect from discontinuities (such as flanges) and flowerpetal apertures can become resonantly trapped in an oversized (multimode) waveguiding system, resulting in large loss of power from the desired $\mathrm{TE}_{01}$ transmission mode. ${ }^{37}$ The quality factors of these unwanted resonances in the circular waveguide can be reduced by introducing arrays of azimuthal rings made of lossy ceramic, or azimuthal slots, which couple non-TE 01 modes out of the waveguide. The spacing and depth of the azimuthal arrays of rings or slots are critical for determining which modes will be suppressed. The trapped-mode problem so far has arisen in the R\&D program during work with a WC175 transmission guide. The unwanted mode(s) are being identified, and suitable mode suppressors will be designed.

\subsubsection{Pumping Ports}

Vacuum pumping ports for circular waveguide are being developed which utilize azimuthal slots, spaced so as to be transparent to $\mathrm{TE}_{01}$ propagation, to couple the waveguide vacuum to a pumping manifold. Each slotted pumpout will be pumped by a 20 -liter/sec ion pump. The high conductance of 3- to 5-inch diameter circular waveguide makes it possible to maintain good vacuum (below $10^{-7}$ torr) over long runs of circular waveguide. 


\subsubsection{Delay-Line Phase-Length Stabilization}

Each SLED-II pulse compressor will have a pair of 120 -foot-long waveguide delay lines, the phase-lengths of which will have to be controlled to approximately 0.001 inch tolerances in order to keep the SLED-II output power stable, as desired, to $0.1 \%$ in amplitude and $0.1^{\circ}$ of phase. The phase-length of a delay line will be stabilized by thermostatic temperature control $\left(0.001\right.$ inch per $\left.0.1^{\circ} \mathrm{F}\right)$, and by an adjustable non-contacting shorting plunger (a phase shifter) at the end of each delay line. The adjustable shorting plunger will be controlled by a feedback loop that utilizes the phases and amplitudes measured at directional couplers at the SLED-II input and output in order to set and stabilize the phase-length of the delay lines. (See also Section 3.9.1.)

\subsubsection{3-dB Couplers}

SLED-II requires a low-loss four-port 3-dB coupler. Two ports serve as input and output. The delay lines are connected to the other two ports.

The 3- $\mathrm{dB}$ coupler planned for the NLCTA is a WR90 side-wall coupled version, mated to circular waveguide via a flower-petal mode transducer at each port. The loss for splitting or combining power is expected not to exceed the loss of three flower petal transitions, or approximately 1.5\%. The first test of this concept will be performed in Summer 1993 with a magic-tee network and four flower-petal transitions. (The X-band magic-tee was available, whereas a 3-dB side-wall coupler will not be designed and fabricated before late 1993.)

Most of our development work on SLED-II so far has been done using a 3-dB coupler built entirely in WC175 circular waveguide, operating in the TE 01 mode. The loss for splitting or combining power in this device is approximately $8 \%$. The greatest drawback to this device is its size and cost. The coupling slot is 1-m long. The entire device (including gentle "S"-bends that spread the circular waveguides wide-enough apart to accomodate flanges on the ports) is 3-m long. An improved and shorter version is being designed in WC165 with a total length of $1.8 \mathrm{~m}$. The $\mathrm{TE}_{01} 3-\mathrm{dB}$ coupler is being considered as a back-up in case the WR90 version proves to have insufficient power-handling capability (which may be the case when two 100MW klystrons are combined into one SLED-II in a future NLCTA energy upgrade).

\subsubsection{Waveguide Bends}

Bends in the circular waveguide power transmission lines will be necessary to get power from the klystron to the accelerator, Figure 6 shows $90^{\circ}$ - and $180^{\circ}$-bends in circular waveguide. We have procured and tested bends of this type from General Atomics (San Diego, California). They work well with less than $2 \%$ power loss, and should have great power-handling capability. However, the bending radii are relatively large for accelerator applications $\left(69 \mathrm{~cm}\right.$ for $90^{\circ}$, and $22 \mathrm{~cm}$ for $180^{\circ}$ ).

We are developing much more compact bends based on the flower-petal transition from circular to rectangular waveguide. The compact bends we plan to use for 
circular waveguide are, in essence, standard WR90 bends with flower petal transitions at either end. Power loss in a bend of almost any angle is expected not to exceed the loss of two flower-petal transitions, or approximately $1 \%$.

\subsubsection{Power Divider}

Power from each klystron and SLED-II compressor must be divided to fill two accelerator sections. A power divider has been fabricated and is awaiting testing. It is comprised of a flower-petal transition from circular waveguide to two anti-parallel runs of WR90. 


\subsection{ACCElerator SeCtions}

Sketches of an NLCTA accelerator section and its individual cells are shown in Figures 27 and 28, respectively. Each cell of a section will have different cavity diameter, iris diameter, and iris wall thickness. All dimensions will be calculated to give the required dispersion characteristics for the fundamental mode and for the higher-order dipole modes, as described above.

Six cells (one close to each end, and four evenly distributed between them) will have radial pumping holes connecting the central (beam) cavity to two outer vacuum manifolds. These manifolds will each be connected to two 8 -liter/s vacuum pumps in the middle of the section. The manifolds will increase the pumping speed through the small beam apertures by an order of magnitude. This improvement is considered necessary to handle the increased gas load resulting from high peak power rf propagating through the structure.

Four water-cooling tubes will be brazed along the outside of cach scction, as shown in Figure 27. The outer surface of each cell can be used for precise support and alignment since it is concentric to the iris diameter to within 5-10 $\mu \mathrm{m}$. Two tuning stubs will be brazed into each cell wall to permit fine tuning of the phase advance per cell of a completed section. Symmetrical double-input couplers will be used at the ends of each section to minimize the phase and amplitude asymmetries in the coupler fields.

Each accelerator section will be mounted on an aluminum strongback. Supports from the strongback to the section will be positioned under the pumping cells, as shown in Figure 27. The support at the input-coupler end will be rigidly attached to the strongback. All other supports will be flexible to allow for longitudinal differential expansion. Each support will permit vertical fine adjustment so that the section can be held straight to within $5 \mu \mathrm{m}$. 


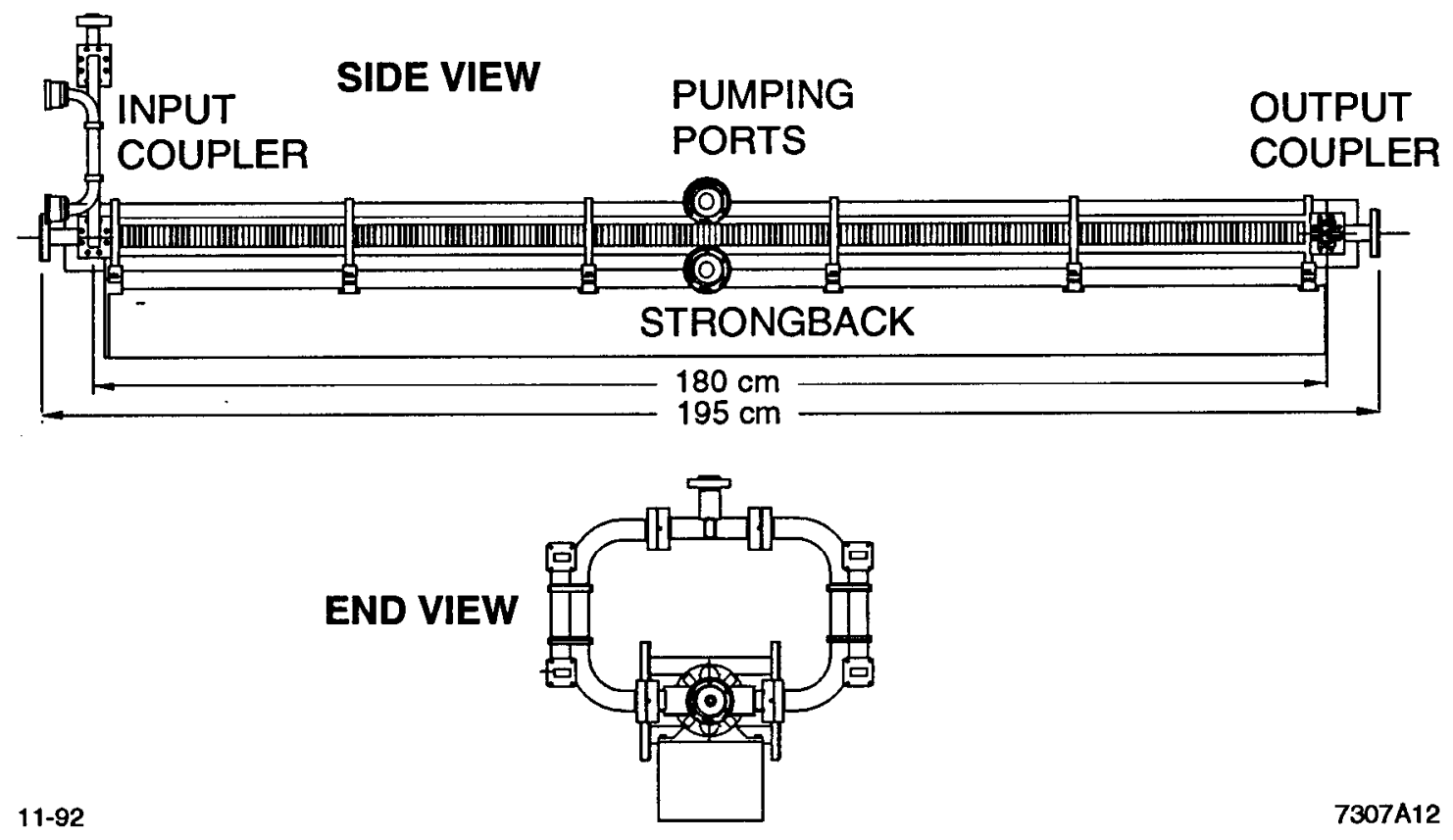

Figure 27. A 1.8-m-long NLCTA accelerator section. 


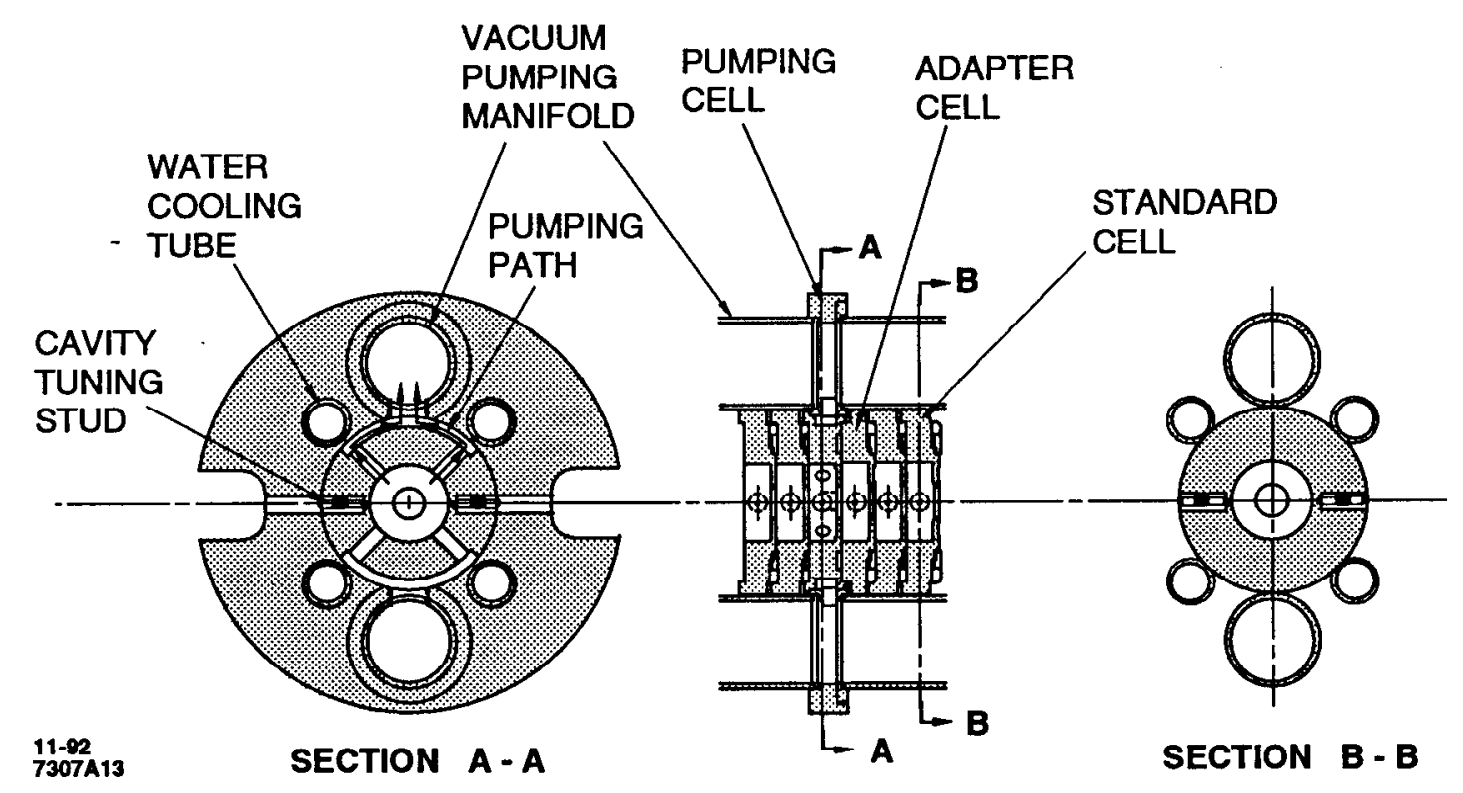

Figure 28. Cells of an NLCTA accelerator section. 


\subsection{Protection Systems}

The protection systems are divided into three groups. The Beam Containment System (BCS) will insure that the confinement integrity of the beam line housing is not violated by large excursions of the beam from its nominal trajectory. The Personnel Protection System (PPS) will insure the safety of personnel in and around the NLCTA beam line housing by controlling access to the housing, by providing emergency-off controls, and by monitoring radiation levels via Beam Shutoff Ion Charmbers (BSOICs). Any PPS or BCS violation will result in the inhibiting of redundant permissives to the associated systems. Finally, the Machine Protection System (MPS) will provide for the protection of beam line components and inhibit permissives to the associated systems in the event of a violation.

\subsubsection{Beam Containment System}

The BCS will be modeled on the system that is in use in the SLC. Currently, the SLC system is monitored by personnel in the control room via hard-wired annunciator panels. However, an the engineering redesign of the SLC BCS system has been proposed in which monitoring would be via CAMAC, and control would be through a dedicated hardware interface. If it is approved and completed by the end of FY93, it will be incorporated into the NLCTA.

Six Beam Containment Ion Chambers will be installed at selected locations along the beam line. Their signals will be processed and the results will be sent to a summary interlock module that will permit/inhibit appropriate beam line systems.

\subsubsection{Personnel Protection System}

The PPS Access Control System will have three access states: "Permitted Access", "Controlled Access", and "No Access". Entry to the beam line housing will require the thermionic gun and the modulators in the rf system to be disabled. The PPS system will not provide for the control of electrical hazards. For any access into the NLCTA beam line housing, all non-insulated Class-B hazards will be required to be turned off and secured using lock \& tag procedures under administrative control.

'The entrance point at each end of the beam line housing will have door and gate standard access modules. Both access points will contain an outer door, inner gate, key bank, access annunciator panel, door control boxes, emergency entry/exit device, search reset box, telephone, and TV camera. The outer door will use a magnetic lock which will have an interlock circuit to insure its proper operation. Preset and Emergency-Off boxes will be located in the ESB utility tunnel and on the inner wall of the shielded enclosure.

There will be eight BSOICs installed in and around the housing. If radiation levels exceed a preset threshold, the units will trip and disable the thermionic gun and the modulators in the rf system. 
The PPS Access Control System will be operated from a panel in the NLCTA control room. An additional panel will be located in a PPS back-up rack (behind locked doors), and will be used by the PPS crew for maintenance and certification purposes only. The logic will be designed using fail-safe and redundant relay circuit techniques. All hardware will be housed in locked cabinets and racks. Wires and cables will be protected in conduit, armored cable, or trays.

\subsubsection{Machine Protection System}

The MPS will interface to the SLC control system via SLAC designed CAMAC modules.

Six Protection Ion Chambers (PICs) will be installed near the collimators in the beam line. Their signals will be processed by two five-channel Protection Ion Chamber CAMAC modules. Each module will provide PIC summary information to the control system and will send status signals to the MPS VME system, which will permit/inhibit appropriate beam line systems.

Resistive Temperature Detectors (RTDs) will be installed to monitor temperatures of beam line components. These sensors will be monitored by the MPS Summary Interlock Chassis via a Daytronics industrial process controller unit.

The flow of cooling water to individual components will be monitored through the use of venturis and pressure differential flow switches. The status of these switches will be sent to the MPS VME crate via a Latching Digital Input Module (LDIM), and then to the MPS Summary Interlock Chassis. 


\subsection{Control System Architecture}

The control system for the NLCTA will be an extension of the system that controls the SLC. The SLC VAX computer controls and monitors the machine though a series of distributed microprocessors, which in turn control beam line components via CAMAC. The topology of the system is a logical star network with the Ilost machine coordinating geographically distributed slave microprocessor clusters. The NLCTA will "appear" to the SLC control system as one more microprocessor cluster. The advantage of this arrangement is that we can utilize software and hardware that has been extensively tested in the SLC.

\subsubsection{VAX/Multibus Interface}

The SLC control system is designed to allow for multiple independent control consoles. Most of the control consoles and remote terminals interface to the VAX through SLCNet, a $1 \mathrm{Mb} / \mathrm{sec}$ broad band local area network. However, we will use the faster EtherNet interface which has recently become available at SLAC. EtherNet connection ports will be installed in the control room, beam line housing, and in the electronic rack support area.

Four 19-inch Color workstations will be installed in the control room as control consoles and dedicated displays. These workstations will emulate the SLC control - touch panels, and will have full graphics display capability. An Imagen laser printer will be installed to provide hard-copies of text and graphics.

Communication between the HOST VAX and the microcluster will be through SLCNet using one $6 \mathrm{MHz}$ channel of the SLC CATV cable system. This will require extending the cable system into the rack equipment areas within End Station B.

\subsubsection{Micro System}

'The microcluster system will consist of two Multibus crates each containing an Intel 486/4 MB CPU processor board and support modules. Each Multibus crate will have an individual blower assembly for cooling; if necessary, an air conditioning unit will be installed.

\subsubsection{CAMAC System}

Sixteen CAMAC crates will be connected to the Multibus system through SLAC's high-speed (5 Mbit/sec) Serial Crate Controller module (SCC). Each CAMAC crate will contain a Crate Verifier module, Programmable Delay Unit (PDUII), and Simple Timing Buffer (STBII) as its base configuration.

\subsubsection{Signal Processing}

Signal distribution to the control room will be through a local signal multiplexer, a GPIB/CAMAC interface module, and cables via patch panels. Video signal distribution to remote areas, such as the Main Control Center, will be through the FFNet 
CATV network, which will be extended into the NLCTA equipment rack arca. Two rack mounted video modulator units will provide the capability for video transmission on two channels of FFNet.

To digitize the video signals, a Video Digitizer Clock Interface (VDCI) and Flash Analog to Digital Converter (FADC) module will be installed. These modules are used in the SLC for the same purpose, so the associated control and data analysis software already exists.

For general purpose signal measurements, a high-speed $(400 \mathrm{MHz})$ analog oscilloscope will be rack mounted in the control room.

\subsubsection{Timing System}

Timing signal generation will be provided through the extension of the SLC Main Drive Line (MDL) signal ( $476 \mathrm{MHz}$ ) to the NLCTA equipment rack area. The signal will be amplified and then distributed via the SLAC built SLC FIDO II chassis and signal distribution chassis.

\subsubsection{Interlock Power \& Trunking}

To provide for general interlock capability, two rack mounted $24 \mathrm{VDC} / 25 \Lambda$ power supplies will be installed together with 50-channel distribution panels which contain a fuse for each channel. Rack interconnection for the distribution of interlock power and signals will use multi-conductor trunks terminated on Faston 50-point cross-connect blocks mounted in each double-bay rack. 


\subsection{Support And Alignment}

Although the NLCTA will test the high-gradient linac performance relevant for the NLC, it will not be used to test the alignment and stabilization techniques that must be developed for the NLC. The combination of the short linac, large transverse beam size and small bunch intensity makes the NLCTA beam transport system fairly insensitive to misalignments and vibrations. For example, the alignment of the quadrupoles, beam-position monitors and accelerator structures in the NLC linac will have to be maintained at the $10-\mu \mathrm{m}$ level over distances of several meters to prevent a significant growth of bunch emittance due to wakefields and dispersive effects. Such displacements in the NLCTA will have a negligible effect on the bunch sizes, even if an NLC-like bunch train is injected. Likewise, the effects from mechanical motion, which have micrometer (and smaller) tolerances in the NLC, will not have a discernable effect on the NLCTA bunch sizes. The effect on the transverse bunch positions in the NLCTA will be more pronounced; however, the experimental analyses necessary to isolate the sources are fairly complex and, at best, would not provide the necessary resolution.

For these reasons, we have decided not to make the NLCTA a model of the NLC linac in terms of support and alignment. The Final Focus Test Beam at SLAC will test methods for alignment and stabilization at levels comparable to those required for the NLC main linacs. Instead, for the NLCTA, we will rely on conventional techniques for support and alignment that are used routinely at SLAC.

The NLCTA support system will consist of a series of girders similar to those used in the SLC Final Focus region. In their construction, two steel I-beams will be cast into a rectangular cement block and machined flat. The rail system formed by the I-beams will be used to support the beam line components. The girders themselves will each be supported from the floor by adjustable legs. The vibration amplitude and the diurnal motions of the floor in End Station B are well below the level that will have a significant effect on the beam transport in the NLCTA. Thus, no special pier system or vibrational stabilization will be used although care will be taken to prevent nearby mechanical systems, such as water pumps and flows, from driving the motion of the beam-line elements.

Before installation of the beam line components, they will be fiducialized, i.e. they will have external surveying fixtures mounted on them at precisely known positions relative to their axis. These fiducials will allow the use of both optical tooling and triangulation techniques for component alignment. For the quadrupoles, the positions of their magnetic centers relative to the fiducials will be measured. In addition, measurement offsets of the BPMs will be determined with respect to the magnetic centers of the quadrupoles in which they are mounted. We expect to achieve an accuracy in the quadrupole and BPM fiducialization of better than $100 \mu \mathrm{m}$.

In the shielded enclosure, a monument system will be established to define a global reference system. The elements will be installed and positioned relative to 
this reference system. Each component will be supported by an SLC Final Focusstyle precision adjustment stage. The first alignment iteration will set component positions to the sub-millimeter level for vacuum chamber installation and hook-up. A combination of differential leveling and optical tooling offset measurements will be used to both survey and control component moves. The process will be repeated after vacuum make-up to bring the components on each girder into alignment with each other to within $250 \mu \mathrm{m}$. Triangulation or optical tooling and differential leveling will be used to survey component positions and control moves. Once all components are in place, the entire system will be surveyed using triangulation techniques to make the system "smooth." Component moves will be controlled using digital indicators interfaced with a laptop computer. At least two iterations of survey and alignment will likely be required for this process to converge. One further triangulation survey will verify the final component positions.

For the critical elements, the quadrupole magnets and accelerator structures, this procedure should yield a transverse alignment of about $150 \mu \mathrm{m}$ over distances of 10 meters. Such an alignment will not significantly degrade the bunch emittances during their transport if the corrector dipole magnets are used to keep the bunch centroids "zeroed" in the position monitors. For non-critical components, such a bend magnets, we require only a $500 \mu \mathrm{m}$ alignment, which relaxes the tolerances on their fiducialization. 


\section{Experiments}

\subsection{OVERVIEW}

The goal of the NLCTA experimental program is to measure the performance characteristics of the multi-section X-band linac and high-power if systems. The experiments will go beyond the work with single power sources and structures at the Accelerator Structure Test Area (ASTA) in the Klystron Test Laboratory; the NLCTA beam will be much more intense, will be much better defined, and will be more precisely analyzed, both before and after acceleration. In addition to system operations tests, beam-dynamics studies will be done using three types of bunch trains: a short bunch train of a fcw nanoseconds will be used to measure the unloaded energygain characteristics of the linac, a variable-length bunch train will be used to measure field-emission currents in the linac with and without injected beam, and the nominal 150-ns-long bunch train will be used to test multi-bunch beam-loading compensation. The specifics of these experiments are discussed in the following sections, concluded by a discussion of other types of wakefield studies that will be made possible by upgrading the NLCTA injector. Although we will not elaborate on it further, an important part of the experimental program, of great relevance for future linear colliders, is the commissioning of the multi-bunch beam instrumentation described in Section 3.7.

\subsection{System Studies}

The NLCTA rf system was designed to be large enough in scale, with four klystrons powering eight structures through four SLED-II rf pulse compressors, that it will serve to test system control and monitoring methods applicable to NLC-scale linacs. A key component of the monitoring system will be instrumentation that can detect and digitize the phase and amplitude envelopes of the 250-ns-long rf pulses. This waveform sampling system, which will be based on commercially available highspeed waveform digitizers, is intended to resolve $0.1 \%$ amplitude and $0.1^{\circ}$ phase variations on time-scales longer than a few nanoseconds. The data acquisition will be controlled by local microprocessors, one of which will be assigned to each rf station," in order to permit parallel, high-level processing of the data.

We plan to monitor the transmitted and reflected rf pulses at many points along the route between each klystron and its associated accelerator structures. There will be two waveform digitizers per rf station, into which the rf phase and amplitude signals from the monitor points can be multiplexed. In this way, correlations between any two signals can be examined. An important function of the monitoring system will be to characterize the stability of the rf pulses. For this purpose, the signals

* An rf station is defined as one klystron, its SLED-II pulse compressor, and its pair of accelerator structures. 
will be processed in three basic ways. To characterize the short-term stability of some aspect of the waveform, a few hundred consecutive pulses will be sampled and analyzed. To monitor long-term stability, a sample will be taken every few minutes and stored in history buffers. Finally, to monitor rare occurrences, the system will be set up to continuously sample every pulse, but will save only a portion of the data associated with distinguishable events.

\subsection{UNLOADED ENERGY GAIN}

To measure the unloaded energy gain characteristics of the linac, only one klystron will be run at a time. A short bunch train, which makes the beam loading negligible, will be used to simplify the measurement. The energy gain of the bunch train depends on the rf waveform entering the two energized structures, on the rf transmission properties of the structures, and on any ficld emission (or breakdown) that occurs in the structures. The rf transmission properties of the structures have been computed ${ }^{39}$ so that, from a measurement of the input rf waveform, one can predict how the ideal energy gain should vary as a function of the transit time of the bunch train relative to the rf pulse. By comparing this calculation to measurements dome at low rf-power levels, we will test our theoretical understanding of the rf transmission properties of the accelerator sections. By comparing low- and highpower measurements, we will look for effects from field emission within the structures. Checks for field emission will also be made by observing the energy-gain stability at a fixed transit time for different rf power levels.

\subsection{Linac Field-Emission CurRent}

In addition to over-loading individual structures, there is a concern that field emission currents will be accelerated sufficiently to be captured by the accelerating wave and transported in an NLC-like linac. To investigate this possibility, and to provide more data on field-emission activity in the structures, the NLCTA linac will be instrumented with two types of detectors. An extended scintillation counter will be placed alongside each of the structures to provide a measure of the expelled (uncaptured) field-emission current, and resistive wall monitors will be installed in the beam line at the beginning, middle, and end of the linac to measure the transported current. The time-resolution of the scintillation counters will be approximately $20 \mathrm{~ns}$. The time-resolution of the resistive wall monitor will be approximately $1 \mathrm{~ns}$.

With the beam off, we will measure the expelled and transported current signals as functions of the rf power and of the strengths of the intervening quadrupole and dipole magnetic fields. With a strong dipole field, any wall monitor currents should be due only to field emission in the structure immediately upstream. By observing the change in the currents with no dipole field, but with different-strength quadrupole configurations, we will characterize the transport of field-emission current in the linac. Although the quadrupole field strengths available in the NLCTA will not be as great 
as the strengths that will be used in an NLC main linac, we believe that they will be sufficient to fully suppress the transport of the field-emission current. Studies will also be done to measure how much of the field-emission activity is associated with the presence of the beam by varying the length of the bunch train and observing the change in the time-structure of the monitor signals.

\subsection{Multi-Bunch Beam-Loading Compensation}

One of the goals of the NLCTA is to demonstrate that multi-bunch beamloading compensation can be readily maintained at the $0.1 \%$ level for a beam current yielding a steady-state loading of $25 \%$. To achieve the compensation, the shape and/or timing of the rf pulses will be adjusted so that the resulting change in unloaded energy gain along the bunch train offsets the energy loss from the loading. Demonstrating compensation at the $0.1 \%$ level will require careful preparation of the bunch train so as not to generate other sources of bunch-to-bunch energy differences. The bunch train injected into the linac will be checked for tolerances on the uniformity of bunch energy, intensity, and spacing. To measure the degree of compensation, the bunch train will be analyzed in the spectrometer using a vertical kicker magnet, as described in Section 3.7.2. From the measurements of the rf waveform entering the structures, and from our theoretical understanding of the rf transmission through the structures, ${ }^{39}$ we should be able to predict the gross features of the bunch-to-bunch energy differences that are observed.

The specific method for shaping the rf waveforms has not yet been selected. Although we will probably modulate the amplitude of the rf drive to the klystrons, various types of rf phase modulation prior to SLED-II pulse compression are also options to be tried. The rf must be stable in time, whichever method we choose. Maintaining the stability of the rf pulses may require feedback which should be relatively straightforward to implement in the rf control system that is envisioned for the NLCTA.

\subsection{Transverse Components of the Accelerating Field}

The tolerances on the allowable transverse components of the accelerating field in the structures of an NLC linac are fairly tight compared to SLC standards. These tolerances differ depending on whether the transverse electric field is in-phase or $90^{\circ}$ out-of-phase with the bunches, and on whether the field jitters from pulse to pulse or is static. In the NLCTA, we will be able to measure transverse electric-field components with resolution on the order of, or smaller, than the NLC tolerances.

The basic approach in these measurements is to vary the phase of one klystron at a time and to record the change in the bunch trajectories using the BPMs downstream from the associated pair of structures. Fitting the amplitudes of the observed betatron oscillations to a sinusoidal function of the klystron phase will yield the in-phase and out-of-phase static components of the accelerating field. To measure the jitter in 
these components, the pulse-to-pulse rms variation of the induced betatron motion will be computed with the klystron on and off, and at the in-phase and out-of-phase settings.

To obtain accurate results will require special attention to how the data are taken. A short bunch train will be used, only one linac klystron will be powered at a time, and the quadrupoles will be turned off downstream of the associated structures so that bunch cncrgy changes do not produce trajectory changes. The measured outgoing trajectory will be corrected pulse-to-pulse to account for any changes in the incoming trajectory. This correction will be based on the trajectory fit to the BPM data in the region between the center of the chicane and the structures being powered. With this procedure, a resolution of about $0.2 \mathrm{keV}$ in the transverse accelerating field should be achieved.

The out-of-phase component of the transverse field, which will degrade the bunch emittance in the NLC due to the differential kick it produces along the length of the bunch, should be less than $1 \mathrm{keV}$ to keep the emittance growth below $10 \%$. Therefore both static and jitter components of this magnitude should be discernable in the NLCTA. Jitter in the in-phase component of the transverse field will also lead to emittance growth in the NLC, as a result of the dispersion it generates. The tolerance for a $10 \%$ growth is much smaller, about $0.2 \mathrm{keV}$, so we will not be able to put tight limits on the NLC emittance growth from this mechanism if indeed no jitter is observed.

A static in-phase component of the transverse field is fairly benign since its effect can be offset with a dipole magnet. Coupled with jitter in the energy gain, however, it also contributes to the emittance growth in the same manner as in-phase jitter. A $1 \%$ jitter in energy gain, which is reasonable for the klystrons being developed, yields a tolerance 100 times larger, or $20 \mathrm{keV}$, on the static in-phase component. Our resolution for measuring this component is also much larger since an absolute measure of the bunch angular trajectories relative to the structure axes are needed to correct the measurement for the transverse field component due to off-angle trajectories. For this correction, we will rely on the absolute BPM calibration and on the BPM alignment to the structure axes, each of which will be known to about $100 \mu \mathrm{m}$. These errors translate into a $10-\mathrm{keV}$ uncertainty in the static field strength so, again, the measurement resolution is near the tolerance.

\subsection{Transverse Multi-Bunch Beam Breakup}

The NLCTA (like the NLC) would not operate stably if conventional constant impedance accelerator structures were used. In both cases the offset of the bunches would be amplified by many orders of magnitude by the end of the linac. The detuned structures for the NLCTA will eliminate this blowup. This will be tested experimentally using the straight-ahead line of the spectrometer and the vertical kicker magnet.

Using corrector magnets in the chicane, the bunch train will be launched into 
the linac with a horizontal position or angle offset. The resulting positions of the bunches exiting the linac will be measured using a profile monitor in the straightahead line of the spectrometer in conjunction with the vertical kicker. This will allow us to distinguish the bunches along the train by their vertical offset while observing the amplification of the initial horizontal betatron amplitude.

\subsection{STUDIES WITH AN UPGRADED INJECTOR}

There are a number of NLC linac beam-dynamics issues which cannot be readily addressed with the NLCTA as designed. The small bunch intensity produced by the X-band injector makes single-bunch longitudinal- and transverse-wakefield effects too small to be measured easily. Also, the small bunch spacing makes it extremely difficult to accurately measure the strength of the long-range transverse wakefield from its effect on betatron motion. As discussed in Sections 2.1 and 2.7, we plan to upgrade the injector in the future to produce NLC-like bunch trains with $0.65 \times 10^{10}$ electrons per bunch and 1.4-ns bunch spacing. If a photocathode rf gun is used, we may be able to produce an adjustable-length bunch train (from 1 to 100 bunches) with a fairly small normalized (invariant) emittance $\left(\epsilon_{n}<10^{-5} \mathrm{~m}-\mathrm{rad}\right)$ and NLC-like bunch lengths (100 $\mu \mathrm{m}$ after compression).

With such an injector operating in a single-bunch mode, the mean energy of the bunch in the spectrometer would be measured as a function of bunch intensity to infer the short-range longitudinal wakefield strength. The size of this quantity is of particular interest since it has some theoretical uncertainty for such short bunches. Similarly, the strength of the short-range transverse wakefield would be inferred by measuring the change in the transverse bunch profile, using the wire scanner before the spectrometer bend, as a function of bunch intensity and betatron amplitude in the linac. To study the long-range transverse wakefields, a long bunch train would be used and the effects of the wakefields on the betatron motion of the bunches in the linac would be measured.

Until a new injector is built, some of these issues will be addressed with the Accelerator Structure SET-up (ASSET) facility at the SLC. ASSET will be built into the SLC to allow measurements of the wakefield characteristics of a single unenergized accelerator structure. In particular, ASSET is optimizcd to measure the long-range transverse wakefield to verify the suppression expected from the detuning of the X-band accelerator structures, as shown in Figure 9. 


\section{Environment, Safety, and Health}

\subsection{OVERVIEW}

SLAC has numerous environment, safety, and health (ES\&H) programs already in place. From the ES\&H standpoint, the Next Linear Collider Test Accelerator (NLCTA) does not present any significant new challenges. All of the anticipated hazards are ones that SLAC has successfully faced during previous construction and/or experimental activities.

The SLAC programs in ES\&H will ensure that all aspects of the design, installation, testing, and operational phases of the project are properly managed. As appropriate, the cognizant SLAC safety committees, including the Safety Overview Committee, the Hazardous Experimental Equipment Committee, the Radiation Safety Committee, the Fire Protection Safety Committee, the Hoisting and Rigging Committee, the AL $\Lambda$ RA Committcc, the Electrical Safety Committee, the Non-Ionizing Radiation Safety Committee, and the Earthquake Safety Committee will review and approve various aspects of the project. All aspects of the project will conform to the applicable DOE, national, and state codes and regulations, including those aspects of DOE 6430.1A that pertain to the NLCTA.

\subsection{Fire Safety}

The fire protection system, which will be a wet pipe design, will be in accordance with NFPA 13. SLAC subcontracts with the Palo Alto Fire Department to operate an on-site fire station and to provide emergency response services. The Palo Alto Fire Department also provides ongoing fire safety inspections of SLAC facilities, as well as training of personnel.

\subsection{Radiation SAFETY}

The design and operation of all facilities at SLAC are governed by the ALARA (as low as reasonably achievable) policy. Thus, SLAC has always maintained radiation dose limits below the maximum allowed by regulation.

\subsubsection{Radiation Shielding}

Shielding for the NLCTA will conform to the Design and Control section of DOE Order 5480.11, Section $9(\mathrm{~J}){ }^{40}$ The conceptual design of the shielding, described Section 2.10, limits the dose equivalent in occupied areas (including the instrumentation and control racks, klystrons, and modulators) to 1 rem/year at the surface of the shield, under the assumption of relatively large beam losses. However, rep-rate limiting will be employed for further dose reduction while the sources of beam loss are located and corrected. Activation of air, ground, or beamline components will not be significant radiological hazards. 
The dose at the SLAC site boundary, greater than $300 \mathrm{~m}$ away, will be less than $0.1 \mathrm{mrem} /$ year.

\subsubsection{Personnel Protection System}

The Personnel Protection System (PPS) will insure the safety of personnel from radiation hazards in and around the NLCTA beam line housing by controlling access to the housing, by providing emergency-off controls, and by monitoring radiation levels via Beam Shutoff Ion Chambers (BSOICs). Any violation of the PPS will result in the inhibiting of redundant permissives to the associated systems. This will be accomplished through a system of electronically interlocked gates, lights, alarms, and operator displays and controls.

The PPS Access Control System will have three access states: "Permitted Access", "Controlled Access", and "No Access". Entry to the beam line housing will require the thermionic gun and the modulators in the rf system to be disabled. The PPS system will not provide for the control of electrical hazards. For any access into the NLCTA beam line housing, all non-insulated Class-B hazards will be required to be turned off and secured using lock-and-tag procedures under administrative control.

The entrance point at each end of the beam line housing will have door and gate standard access modules. Both access points will contain an outer door, inner gate, key bank, access annunciator panel, door control boxes, emergency entry/exit device, search reset box, telephone, and TV camera. The outer door will use a magnetic lock which will have an interlock circuit to insure its proper operation. Preset and Emergency "crash-off" boxes will be located in the ESB utility tunnel and on the inner wall of the shielded enclosure.

There will be eight BSOICs installed in and around the housing. If radiation levels exceed a preset threshold, the units will trip and disable the thermionic gun and the modulators in the rf system.

The PPS Access Control System will be operated from a panel in the NLCTA control room. An additional panel will be located in a PPS back-up rack (behind locked doors), and will be used by the PPS crew for maintenance and certification purposes only. The logic will be designed using fail-safe and redundant relay circuit techniques. All hardware will be housed in locked cabinets and racks. Wires and cables will be protected in conduit, armored cable, or trays.

\subsubsection{Beam Containment System}

The Beam Containment System (BCS) will insure that the confinement integrity of the beam line housing is not violated by large excursions of the beam from its nominal trajectory. Any BCS violation will result in the inhibiting of redundant permissives to the associated systems.

The BCS will be modeled on the system that is in use in the SLC. Currently, the SLC system is monitored by personnel in the control room via hard-wircd annunciator 
panels. However, an engineering redesign of the SLC BCS system has been proposed in which monitoring functions would be performed via CAMAC, and control functions would be performed through a dedicated hardware interface. If it is approved and completed by the end of FY93, it will be incorporated into the NLCTA.

Six Beam Containment Ion Chambers will be installed at selected locations along the beam line. Their signals will be processed and the results will be sent to a summary interlock module that will permit/inhibit appropriate beam line systems.

\subsubsection{Radiation Safety Training}

In accordance with SLAC's implementation plan for DOE Order 5480.11 (Radiation Protection for Occupational Workers) and the SLAC Radiological Control Manual, all SLAC employees and any persons who work at the laboratory longer than one month must receive training in radiation fundamentals through General Employee Radiological Training. In addition, those workers whose assignments make it likely that they will receive a total occupational radiation dose greater than 100 mrem in one year receive more extensive radiation safety training and are classified as Radiation Workers. Both classes of workers must be recertified every two years.

\subsection{High-Power Microwave System Safety}

The rf system for the NLCTA will incorporate all the safety measures that are currently in place for high-power microwave systems at SLAC.

All high-power microwave radiation will be confined in evacuated metallic waveguides. The source of high-power microwaves will be disabled by hardware interlock within milliseconds of any failure of the vacuum integrity of a waveguide.

The NLCTA operations staff will conduct radiation hazard surveys periodically to ensure that the klystron amplifiers are appropriately shielded.

\subsection{Electrical Safety}

It is SLAC policy that every necessary precaution is taken in the performance of work to protect all persons on the site from the risk of electrical shock and to minimize the probability of damage to property due to electrical accidents. This policy is implemented by assigning responsibility and adhering to basic safety principles, as stated in the Environment, Safety, and Health Manual, and by complying with regulations and procedures appropriate to each operation. Appropriate electrical safety training courses are provided by the Laboratory for those workers who are likely to be exposed to high-voltage hazards.

Several NLCTA subsystems, such as the klystron pulse modulators, will employ high voltages. The controls and work procedures necessary to ensure safe work on these systems are well understood. The provisions for locking of these systems will utilize SLAC's established procedures for lockout and tagout. Energized equipment 
will be worked on only under very limited and controlled conditions, and only qualified employees will perform such work. All work will be performed in accordance with safe work practices and in accordance with OSHA 1910, Subpart S.

Inside the NLCTA beam line housing, insulated covers and barriers will be used to mitigate all potential Class-B electrical hazards to Class-B1. Lock-and-tag procedures will be used for any work that interferes with these protective barriers.

\subsection{Construction}

The line organization acting through the subcontract administrator has primary responsibility for overseeing safety compliance by construction subcontractors. This responsibility includes:

- Apprising subcontractors of SLAC and DOE safety criteria prior to construction,

- Conducting periodic inspections of subcontractor construction areas to evaluate the quality of the subcontractor's safety compliance program,

- Receiving subcontractor accident reports and compiling information for reporting to DOE.

The Quality Assurance and Compliance Department of the ES\&H Division oversees the QACD Subcontractor Oversight Program. ${ }^{43}$

\subsection{EMERGENCY PREPAREdNESS}

Like all experimental equipment at SLAC, the NLCTA will be designed, constructed, and operated in a manner that minimizes the risk of injury to property or personnel as a result of a natural disaster or other emergency situation. In the event of any abnormal condition, the interlock system will automatically shut the machine down until the situation is diagnosed and corrected. The formal emergency planning

system described in the SLAC Emergency Preparedness Plan ${ }^{44}$ will help to ensure a logical, organized, and efficient response to any emergency. It sets forth specific stcps to deal with various emergency conditions, identifies the appropriate personnel to act as resources, and provides a chain of command for responding to unplanned events.

The emergency situation most likely to arise at SLAC is an earthquake. Buildings and structures at SLAC are designed to withstand the effects of a major earthquake. In addition, all mechanical components of the NLCTA will be secured to protect persons working nearby. This will be assured by a review of the design and installation of the experimental equipment by the SLAC Earthquake Committee, as mandated by the SLAC Safety Program. Further, as with all activities at SLAC, operation of the NLCTA will be covered by the SLAC Emergency Preparedness Plan, which outlines the procedures to be followed in the event of an earthquake severe enough to causc possible structural damage or personal injury. 


\subsection{EnVironmental Protection}

\subsubsection{Refurbishing of End Station B}

Making room for the NLCTA in End Station B requires the removal of magnets, detectors, and other valuable material stored in the End Station. Materials to be removed will be surveyed and will be handled in a manner appropriate to the level of residual radioactivity present, if any. Any scrap will be disposed of in accordance with approved procedures.

\subsubsection{Ongoing Environmental Protection Activities}

Construction and operation of the NLCTA is not expected to cause any adverse impact on the groundwater. Preservation of groundwater quality will be ensured through the implementation of the Groundwater Management Program that SLAC is currently preparing to comply with DOE Order 5400.1 .

\subsection{Hazardous Material Issues}

In accordance with 29 CFR 1910.1200 (the OSHA hazard communication standard), SLAC has developed a SLAC Hazard Communication Program. ${ }^{46}$ Under this program, SLAC directs Department Heads and Group Leaders to conduct regular inventories of hazardous materials, to make Material Safety Data Sheets (MSDSs) available to all employees, to ensure appropriate labeling of hazardous materials, to train employees to identify and control hazards in the workplace, and to inform users, subcontractors, and temporary employees of the hazards that may be encountered at SLAC. 


\section{Administration}

\subsection{Project Management}

The NLCTA is organized as an independent project within the framework of the SLAC organization. The project is logically divided into two major parts, each of which is funded differently, at Summary-Level 1 of the Work Breakdown Structure. Part 1 (WBS 1.X) is funded as a capital equipment project and includes all conventional equipment: AC power, LCW, magnets, BPMs, etc. Part 2 (WBS 2.X) is funded with laboratory operating funds and contains the costs to produce the radiofrequency equipment that is developed in the NLCTA rf-system R\&D program. Though they are funded differently, both Parts 1 and 2 of the WBS are to be managed as parts of one project at WBS Summary-Level 0, the NLC Test Accelerator.

Research and development (R\&D) leading to the NLCTA rf system has been in progress for several years and will continue for the duration of the project. The R\&D effort is comprised of three major tasks: development of X-band klystrons, rf pulse compressors, and accelerating structures. Funding for the R\&D program is separate from funding for the NLCTA. The R\&D program and the NLCTA project are linked with milestones which represent points at which decisions are made to fabricate those successful R\&D prototypes that best meet the NLCTA requirements. It is the cost of fabricating NLCTA microwave components, developed in the R\&D program, that is estimated in the NLCTA WBS (Part 2).

The NLCTA is managed by a team composed of physicists and engineers from both the Research and Technical Divisions of SLAC. Each member of the Projcct Management Team is responsible for a major aspect of the project. Figure 29 shows the management organization chart for the Project. The division of responsibility is as follows:

- Project Manager. Responsible for technical and administrative control of all design, construction, budgets, schedule and personnel. Also responsible for all R\&D related activities upon which success of the project depends. Administrative control is accomplished through reviews, meetings, and delegation of responsibility to subsystem project managers.

- Injector/Accelerator Structures Systems Physicist. Responsible for the specification, construction, installation and commissioning of the injector up to the entrance to the chicane and for all accelerator structures including the WR90 waveguide feeding the structure and all instrumentation to monitor rf.

- Pulse-Compression System Physicist. Responsible for the specification, construction, installation and commissioning of all waveguide from the window at the klystron output, including SLED-II and rf transmission up to the WR90 feeding the accelerator structure. This includes all instrumentation necessary to monitor the rf. 


\section{NLCTA Organization}

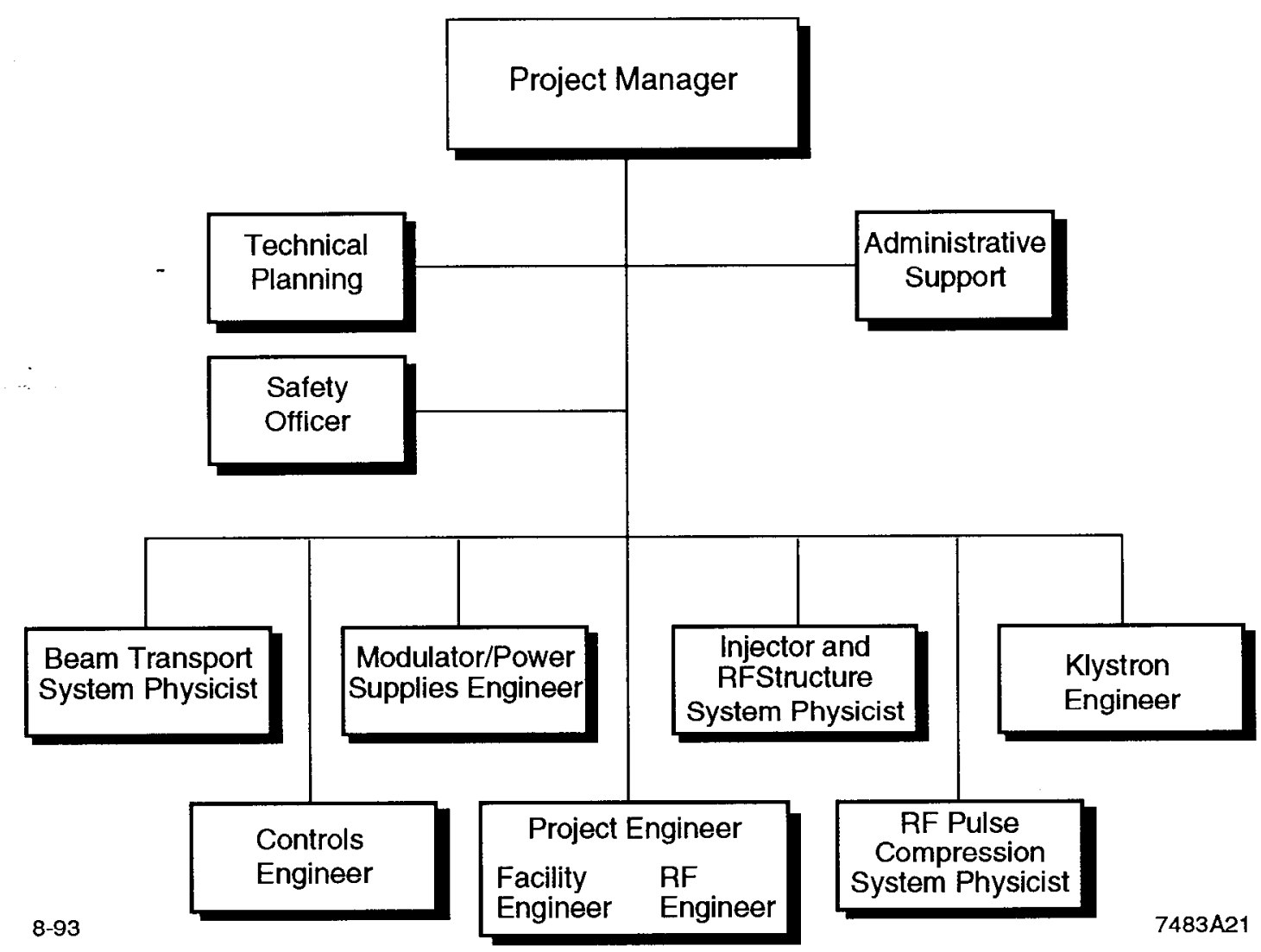

Figure 29. Management organization chart for the NLCTA Project. 
- Beam Transport System Physicist. Responsible for the specification, construction, installation and commissioning of all beamline components from the end of the injector through the spectrometer. This excludes all RF components but includes all instrumentation in this region.

- Project Engineering. Coordinates and oversees all engineering on the NLCTA, including documentation and installation. This function is filled by three positions

- A Project Engineer who works with the Project Manager to define engineering priorities, budgets, and schedules.

- A Mechanical Engineer who is primarily responsible for the conventional facility and the conventional beamline elements in Part 1 of the WBS.

- An RF Engineer who is responsible for microwave engineering of the RF System in Part 2 of the WBS.

The Project Engineering Staff also works with the system physicists to facilitate systems integration and to procure the NLCTA hardware.

- Controls Engineer. Primary liaison to the Controls Department. Works with all system physicists and all engineers to specify the NLCTA Control System.

- Klystron Engineer. Primary liaison to the Klystron Department. Works with system physicists and engineers to ensure that the klystrons meet performance specifications, and that their production and testing is matched to the NLCTA installation and commissioning schedule.

- Modulator \& Power Supply Engineer. Primary liaison to the Power Conversion Department. Responsible for development of the klystron modulators and for procurement of power supplies.

- Safety Officer. Liason to SLAC Safety Committees. Responsible for coordinating Preliminary and Final Safety Analysis Documents.

- Technical Planning. Works with Project Manager and Project Engineer to develop and maintain project schedules, WBS, and CPNs. Also provides spending summaries to track costs on a monthly basis.

Each R\&D effort is led by the NLCTA system physicist or engineer with responsibility for managing the related NLCTA system: the Accelerator Structures System Physicist leads the Structure R\&D effort, the RF Pulse-Compression System Physicist leads the Pulse-Compression R\&D effort, the Klystron System Engineer leads the Klystron R\&D effort.

The Project Management Team meets bi-weekly to discuss project related priorities and key issues which may impact progress. It also holds a general weekly meeting with all project personnel in which global issues are reviewed and discussed. 
Meetings are documented with published meeting minutes. Additionally, the Engineering Group produces monthly engineering progress reports. The management group also publishes a series of NLCTA Notes documenting procedures, specifications and solutions to technical problems.

Schedules for the NLCTA project which integrate WBS Parts 1 and 2 have been compiled. A schedule has been developed for each major subsystem at Level 3, for both WBS numbers. A schedule has also been developed for the R\&D program. The R\&D schedule is linked via milestones to the NLCTA Project subsystem schedules. Sub-system schedules are, in turn, linked together in a master installation schedule for the entire project. 


\subsection{MANPOWER}

The Project Management Team described in Section 6.1 is composed of physicists, engineers, and other professional staff dedicated to the NLCTA Project.

A Mechanical Design Group assigned to the project for its duration will produce most of the design documentation for the entire project, and will perform documentation-control duties.

Most of the hardware, both mechanical and microwave, will be manufactured at SLAC in the shops of the Mechanical Fabrication Department and the Klystron and Microwave Department. Parts for some components, such as accelerator sections, will be machined by off-site shops, and then acceptance tested for quality assurance at SLAC prior to assembly.

Conventional facilities and control systems will be designed and engineered by individuals assigned to the project by the SLAC Plant Engineering, Power Conversion, and Controls Departments.

Construction of the conventional facilities (such as the beamline enclosure, cable plant, AC power, and water systems) will be accomplished through a combination of T\&M (Time \& Materials) workers supervised by SLAC personnel and also contracts to outside vendors.

Installation of beamline and rf hardware will be performed by a dedicated group of NLCTA technicians, on loan to the project from the SLAC Klystron \& Microwave Department and the Mechanical Fabrication Department. Controls hardware will be installed by technicians from the SLAC Controls Department, supplemented by an electronics technician dedicated to the project.

Because the NLCTA will use the SLC control system, software will be written by the SLAC Accelerator Department's Software Group.

Commissioning of the NLCTA will be performed by scientists and engineers from the NLCTA Project. 


\subsection{Cost Estimate, Work Breakdown, ANd Spending}

The NLCTA cost estimate was generated by SLAC engineers who regularly perform the work being estimated. Estimates are based upon each engineer's understanding of the work required and, in most cases, upon similar recently completed jobs at SLAC. Whenever possible, recent vendor quotes were used for hardware. FY93 shop rates were used for labor. Estimates were individually generated and reviewed, and then budgetary contingencies were assigned by assessing associated risk. Systems which are identical to ones built recently at SLAC, such as magnet power supplies and vacuum systems, have well-documented costs and low risks; consequently, they have been assigned low contingencies of $15 \%$. Tasks which are conceptually well understood, but which have not been substantially engineered before, such as the modulators and the cable plant, have been assigned larger contingencies of $25 \%$. Microwave components, whose manufacture is based upon prototype results from an ongoing and parallel R\&D effort, have also been assigned $25 \%$ contingencies. A contingency of $60 \%$ was assigned to the "RF Experimental Data Acquisition" subsystem because SLAC has accumulated very little experience so far with VXI instrumentation systems.

The-cost estimates have been compiled into a Work Brcakdown Structure (WBS), in accordance with DOE document 4700.1 II-9, Part B. The WBS, at Summary-Level 3, is shown in Table 14. The WBS will be used to manage the project; it defines the basic structure for planning, monitoring costs, scheduling, and measuring performance. The WBS is divided into two parts at Level 1. Part 1 covers costs for all conventional project elements where existing technology is being exploited, such as conventional facilities (electrical power, water, shielding, etc.), and conventional accelerator components (magnets, wire scanners, etc.). Part 2 contains the costs for all technologically new items, such as the X-band rf components (including accelerator sections), and the pulse modulators.

Device-specific elements, such as control modules, supports and cables, are included in the WBS category for each particular device. Global items, such as girders, racks, CAMAC crates, and beamline and rf device installation, are given their own WBS summary levels. Costs for ED\&I (Engineering, Design \& Inspection) and B\&H (Building \& Hardware) are consistently broken out at Level 4 . Thus, the levels are defined as follows:

$\begin{array}{lll}\text { Level 0: } & & \text { Project } \\ \text { Level 1: } & \text { X } & \text { Major systems } \\ \text { Level 2: } & \text { X.X } & \text { Major subsystems } \\ \text { Level 3: } & \text { X.X.X } & \text { Major subsystem elements } \\ \text { Level 4: } & \text { X.X.X.X } & \text { ED\&I and B\&H separately } \\ \text { Level 5: } & \text { X.X.X.X.X } & \text { Minor subsystems } \\ \text { Level 6: } & \text { X.X.X.X.X.X } & \text { Minor subsystem detail }\end{array}$

Table 14 shows the total estimated construction cost for the NLCTA Project at Summary-Level 3. All costs are given in FY93 dollars. 
The annual spending profile for the entire project is shown to Summary-Level 2 in Table 15. Yearly spending totals are shown with and without inflationary escalation, which has been estimated at $5 \%$ per year. The contingencies used in the spending profile are averages for each major subsystem. 
Table 14. Work Breakdown Structure (page 1 of 4)

\begin{tabular}{|c|c|c|c|c|c|}
\hline & & $\begin{array}{l}\text { Estimated Cost } \\
\text { (FY93 k\$) }\end{array}$ & $\begin{array}{l}\text { Con- } \\
\text { tin- } \\
\text { gency }\end{array}$ & \multicolumn{2}{|l|}{$\begin{array}{c}\text { Cost + Contingency } \\
(\text { FY93 k } \$)\end{array}$} \\
\hline \multicolumn{2}{|c|}{ Project Total } & 19467 & & 23800 & \\
\hline 1. & Facilities & 10453 & & 12390 & \\
\hline 1.1 & Conventional Facilities & 2961 & $18 \%$ & 3482 & \\
\hline 1.1.1 & ESB Refurbish \& Prep. & & $15 \%$ & & 380 \\
\hline 1.1 .2 & Shielded Enclosure* & & $15 \%$ & & 952 \\
\hline 1.1 .3 & Control Room & & $25 \%$ & & 38 \\
\hline 1.1.4 & AC Power & & $15 \%$ & & 600 \\
\hline 1.1 .5 & LCW, Sprinklers \& Air & & $15 \%$ & & 438 \\
\hline 1.1 .6 & Cable Plant & & $25 \%$ & & 913 \\
\hline 1.1 .7 & Support Girders & & $15 \%$ & & 161 \\
\hline 1.2 & Source & 294 & $25 \%$ & 367 & \\
\hline 1.2 .1 & Thermionic Gun & & $25 \%$ & & 367 \\
\hline 1.3 & Vacuum & 431 & $15 \%$ & 496 & \\
\hline 1.3.1 & Vacuum System & & $15 \%$ & & 496 \\
\hline 1.4 & Magnets & 588 & $16 \%$ & 682 & \\
\hline 1.4 .1 & Quadrupoles & & $15 \%$ & & 350 \\
\hline 1.4 .2 & Dipoles & & $15 \%$ & & 45 \\
\hline 1.4 .3 & Correctors & & $15 \%$ & & 48 \\
\hline 1.4 .4 & Solenoids & & $15 \%$ & & 143 \\
\hline 1.4 .5 & Kicker & & $25 \%$ & & 58 \\
\hline 1.4 .6 & Thermal Protection & & $15 \%$ & & 38 \\
\hline 1.5 & Magnet Power Supplies & 804 & $16 \%$ & 935 & \\
\hline 1.5 .1 & Quadrupoles & 3 & $15 \%$ & & 352 \\
\hline 1.5 .2 & Dipoles & 2 & $15 \%$ & & 32 \\
\hline 1.5 .3 & Correctors & 1 & $15 \%$ & & 138 \\
\hline 1.5 .4 & Solenoids & 2 & $15 \%$ & & 279 \\
\hline 1.5.5 & Kicker & 1 & $25 \%$ & & 134 \\
\hline
\end{tabular}

*Does not include general-purpose concrete shielding blocks capitalized in 1992 at a cost of $\$ 538 \mathrm{k}$. 
Continuation of Table 14. WBS (page 2 of 4)

\begin{tabular}{|c|c|c|c|c|}
\hline & . & $\begin{array}{l}\text { Estimated Cost } \\
\text { (FY93 k\$) }\end{array}$ & $\begin{array}{c}\text { Con- } \\
\text { tin- } \\
\text { gency }\end{array}$ & $\begin{array}{c}\text { Cost }+ \text { Contingency } \\
(\text { FY93 k\$) }\end{array}$ \\
\hline 1.6 & Beam Devices & 2048 & $21 \%$ & 2486 \\
\hline 1.6.1 & Beam Position Monitors & 742 & $25 \%$ & 928 \\
\hline 1.6 .2 & Profile Monitors & 320 & $15 \%$ & 368 \\
\hline 1.6 .3 & Wire Scanners & 296 & $15 \%$ & 340 \\
\hline 1.6.4 & Toroids & 72 & $15 \%$ & 83 \\
\hline 1.6 .5 & Faraday Cup & 58 & $15 \%$ & 67 \\
\hline 1.6 .6 & Collimator & 451 & $25 \%$ & 564 \\
\hline 1.6.7 & Beam Dumps & 109 & $25 \%$ & 136 \\
\hline 1.7 & Protection Systems & 484 & $25 \%$ & 605 \\
\hline 1.7 .1 & Beam Containment & 84 & $25 \%$ & 105 \\
\hline 1.7 .2 & Personnel Protection & 287 & $25 \%$ & 359 \\
\hline 1.7 .3 & Machine Protection & 113 & $25 \%$ & 141 \\
\hline 1.8 & Controls & 655 & $25 \%$ & 820 \\
\hline 1.8 .1 & Sys. Eng'ing \& Integrat'n & 257 & $25 \%$ & 321 \\
\hline 1.8 .2 & Vax/Multibus Interface & 56 & $25 \%$ & 70 \\
\hline 1.8 .3 & Micro System & 50 & $25 \%$ & 63 \\
\hline 1.8 .4 & Camac System & 160 & $25 \%$ & 200 \\
\hline 1.8 .5 & Signal Processing & 51 & $25 \%$ & 64 \\
\hline 1.8 .6 & Timing System & 31 & $25 \%$ & 39 \\
\hline 1.8 .7 & Int'lock Power \& Trunking & 50 & $25 \%$ & 63 \\
\hline 1.9 & Installation & 1572 & $15 \%$ & 1808 \\
\hline 1.9.1 & Alignment & 259 & $15 \%$ & 298 \\
\hline 1.9 .2 & Mech. Sys. Instal'n Support & 1313 & $15 \%$ & 1510 \\
\hline 1.10 & Management & 617 & $15 \%$ & 709 \\
\hline 1.10 .1 & Staff & 575 & $15 \%$ & 661 \\
\hline 1.10 .2 & Materials \& Supplies & 42 & $15 \%$ & 48 \\
\hline
\end{tabular}

Continued on next page ... 
Continuation of Table 14. WBS (page 3 of 4)

\begin{tabular}{|c|c|c|c|c|}
\hline & . & $\begin{array}{l}\text { Estimated Cost } \\
\qquad \text { (FY93 k\$) }\end{array}$ & $\begin{array}{c}\text { Con- } \\
\text { tin- } \\
\text { gency }\end{array}$ & $\begin{array}{c}\text { Cost + Contingency } \\
(\text { FY93 k\$) }\end{array}$ \\
\hline 2. & RF System & 9014 & & 11419 \\
\hline 2.1 & RF Drive System & 1107 & $15 \%$ & 1274 \\
\hline 2.1.1 & Traveling Wave Tubes & 19 & $15 \%$ & 219 \\
\hline 2.1 .2 & Microwave Electronics & 91 & $15 \%$ & 1055 \\
\hline & Pulse Modulators & 879 & $25 \%$ & 1100 \\
\hline 2.2 .1 & Modulator System & 58 & $25 \%$ & 73 \\
\hline 2.2 .2 & Pulse Transformer Tank & 11 & $25 \%$ & 145 \\
\hline 2.2 .3 & Pulse-Forming Network & 38 & $25 \%$ & 476 \\
\hline 2.2 .4 & Power Supply & 22 & $25 \%$ & 286 \\
\hline 2.2 .5 & Modulator Control Electronics & 96 & $25 \%$ & 120 \\
\hline & Klystrons & 1140 & $25 \%$ & 1426 \\
\hline 2.3 .1 & Klystron Acquisition & 93 & $25 \%$ & 1168 \\
\hline $2: 3.2$ & RF Monitoring \& Protection & 15 & $25 \%$ & 193 \\
\hline 2.3 .3 & Klystron Power Supplies & 52 & $25 \%$ & 65 \\
\hline & Pulse Compr'n \& Transm'n & 1495 & $25 \%$ & 1868 \\
\hline 2.4.1 & SLED-II Pulse Compressors & 72 & $25 \%$ & 904 \\
\hline 2.4 .2 & Waveguide Transm'n Sys. & 60 & $25 \%$ & 755 \\
\hline 2.4 .3 & Vacuum System Hardware & 16 & $25 \%$ & 209 \\
\hline & Accelerator Structure & 1923 & $25 \%$ & 2404 \\
\hline 2.5 .1 & Accelerator Sections & 88 & $25 \%$ & 1111 \\
\hline 2.5 .2 & Microwave Feeds & 42 & $25 \%$ & 535 \\
\hline 2.5 .3 & High Power Loads & 11 & $25 \%$ & 145 \\
\hline 2.5 .4 & Prebuncher Cavity & 11 & $25 \%$ & 144 \\
\hline 2.5 .5 & Accel. Vac. Sys. Hardware & 32 & $25 \%$ & 403 \\
\hline 2.5 .6 & Automated Bake-out Equip. & 53 & $25 \%$ & 66 \\
\hline
\end{tabular}


Continuation of Table 14. WBS (page 4 of 4)

\begin{tabular}{|c|c|c|c|c|}
\hline & & $\begin{array}{l}\text { Estimated Cost } \\
\text { (FY93 k\$) }\end{array}$ & $\begin{array}{l}\text { Con- } \\
\text { tin- } \\
\text { gency }\end{array}$ & $\begin{array}{c}\text { Cost + Contingency } \\
(\text { FY93 k } \$)\end{array}$ \\
\hline 2.6 & RF Exp'tal Data Acquisition & 731 & $60 \%$ & 1169 \\
\hline 2.6 .1 & Hardware & 429 & $60 \%$ & 686 \\
\hline 2.6 .2 & Software & 302 & $60 \%$ & 483 \\
\hline 2.7 & RF-Related Beam Inst'ment'n & 345 & $25 \%$ & 433 \\
\hline 2.7 .1 & Wall Current Monitors & 96 & $25 \%$ & 120 \\
\hline 2.7 .2 & Bunch Length Monitor & 76 & $25 \%$ & 95 \\
\hline 2.7 .3 & X-band Beam Phase Mon. & 32 & $25 \%$ & 40 \\
\hline 2.7 .4 & Fast PLIC & 34 & $25 \%$ & 43 \\
\hline 2.7 .5 & Scintillators & 25 & $25 \%$ & 31 \\
\hline 2.7 .6 & Fast (VXI) Electronics & 83 & $25 \%$ & 104 \\
\hline 2.8 & RF System Controls & 529 & $25 \%$ & 662 \\
\hline 2.8 .1 & RF Controls & 439 & $25 \%$ & 549 \\
\hline 2.8 .2 & Vacuum System Controls & 90 & $25 \%$ & 113 \\
\hline 2.9 & Cable Plant & 220 & $25 \%$ & 275 \\
\hline 2.9 .1 & RF Drive System & 25 & $25 \%$ & 31 \\
\hline 2.9 .2 & Pulse Modulators & 24 & $25 \%$ & 30 \\
\hline 2.9 .3 & Klystrons & 30 & $25 \%$ & 38 \\
\hline 2.9 .4 & RF Pulse Compression Sys. & 26 & $25 \%$ & 33 \\
\hline 2.9 .5 & Accelerator Structure & 52 & $25 \%$ & 65 \\
\hline 2.9 .6 & RF System Controls & 54 & $25 \%$ & 68 \\
\hline 2.9 .7 & RF Exp'tal Data Acquisition & 8 & $25 \%$ & 10 \\
\hline 2.10 & Project Installation & 646 & $25 \%$ & 808 \\
\hline 2.10 .1 & RF System Support & 646 & $25 \%$ & 808 \\
\hline
\end{tabular}


Table 15. Annual spending profile for the project

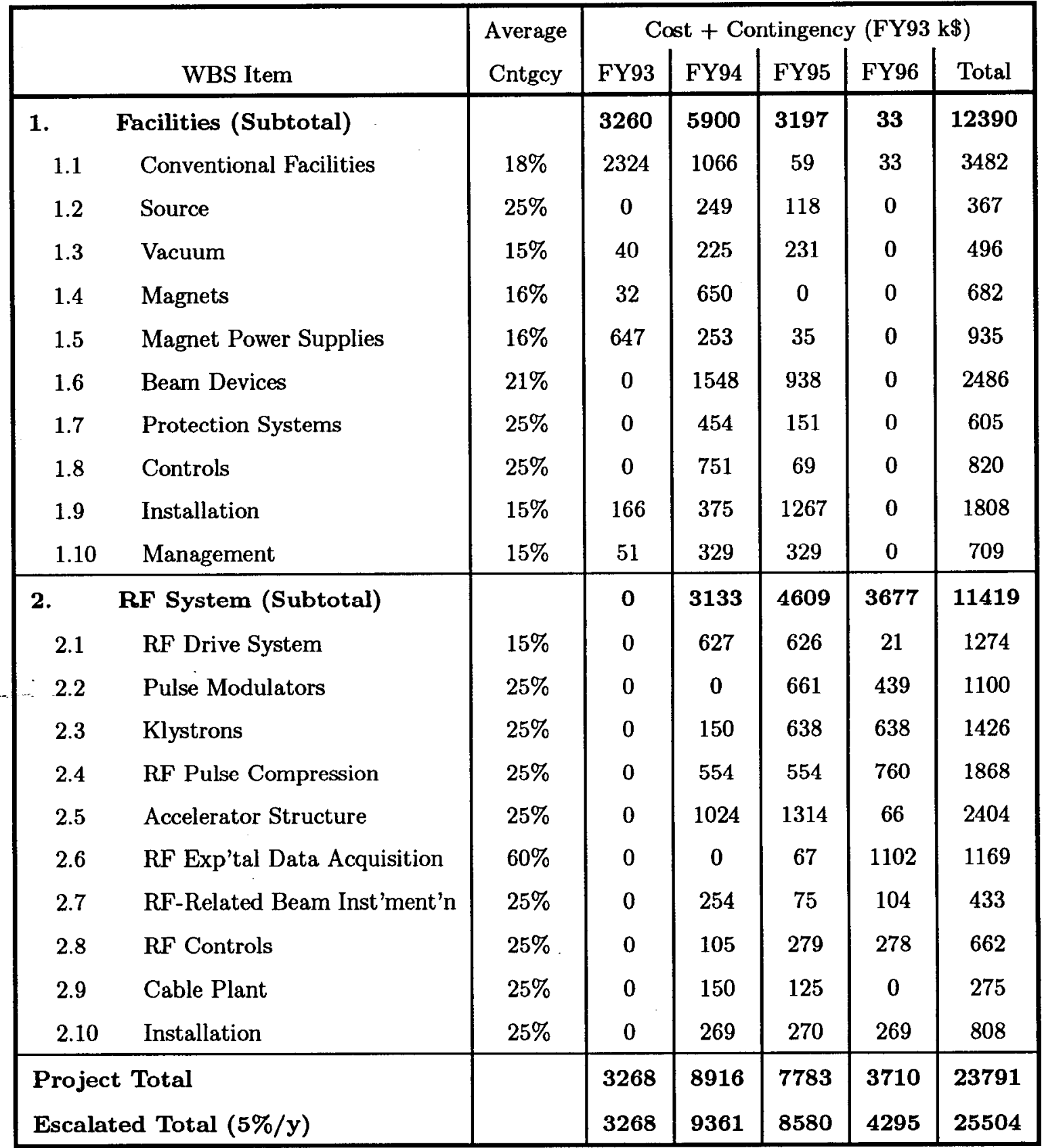




\subsection{SCHEDULE}

Project milestones are shown in Table 16. The schedule for project-related R\&D is shown in Figure 30. The schedule for the project itself, to WBS-Level 3, is shown in Figure 31.

\section{Table 16. Project Milestones}

$\begin{array}{rl}\text { Date } & \text { Milestone } \\ 4 / 91 & \text { Project conceived } \\ 12 / 92 & \text { First project review } \\ 1 / 93 & \text { NEPA approval obtained } \\ 8 / 93 & \text { Conceptual Design complete } \\ 2 / 94 & \text { Preliminary Safety Analysis Document (PSAD) complete } \\ 4 / 94 & 1.8 \text {-m structure prototype tests complete } \\ 4 / 94 & \text { Final klystron prototype complete } \\ 8 / 94 & \text { SLED-II prototype tests complete } \\ 5 / 95 & \text { Injector beamline complete } \\ 6 / 95 & \text { Chicane beamline complete } \\ 6 / 95 & \text { Final Safety Analysis Document (FSAD) complete } \\ 8 / 95 & \text { Linac beamline complete } \\ 8 / 95 & \text { Spectrometer beamline complete } \\ 11 / 95 & \text { Accelerator Readiness Review (ARR) complete } \\ 12 / 95 & \text { Control System complete } \\ 4 / 96 & \text { RF System complete } \\ 5 / 96 & \text { Test beam to end of spectrometer } \\ 6 / 96 & \text { Project complete }\end{array}$

Work has been scheduled such that the beamline enclosure, equipment racks, cablc trays, water systems and AC power will be installed and completed first. Also during this period, beamline girders will be installed and aligned. This will be followed by installation of all key beamline devices, rough alignment and then connection of components that complete the vacuum envelope. Because of its availability and also its sensitivity, the last mechanical hardware to be installed will be the rf system hardware. Controls hardware for the entire system will be installed as it bccomcs available and system checkout accomplished as allowed by the installation of both the mechanical and controls hardware. Since the cable plant will not be ready for installation until midway through the project, it must be installed in parallel with mechanical beamline hardware. This will be accomplished by separating the two tasks into shifts; a day shift for one installation and a night shift for the other. 


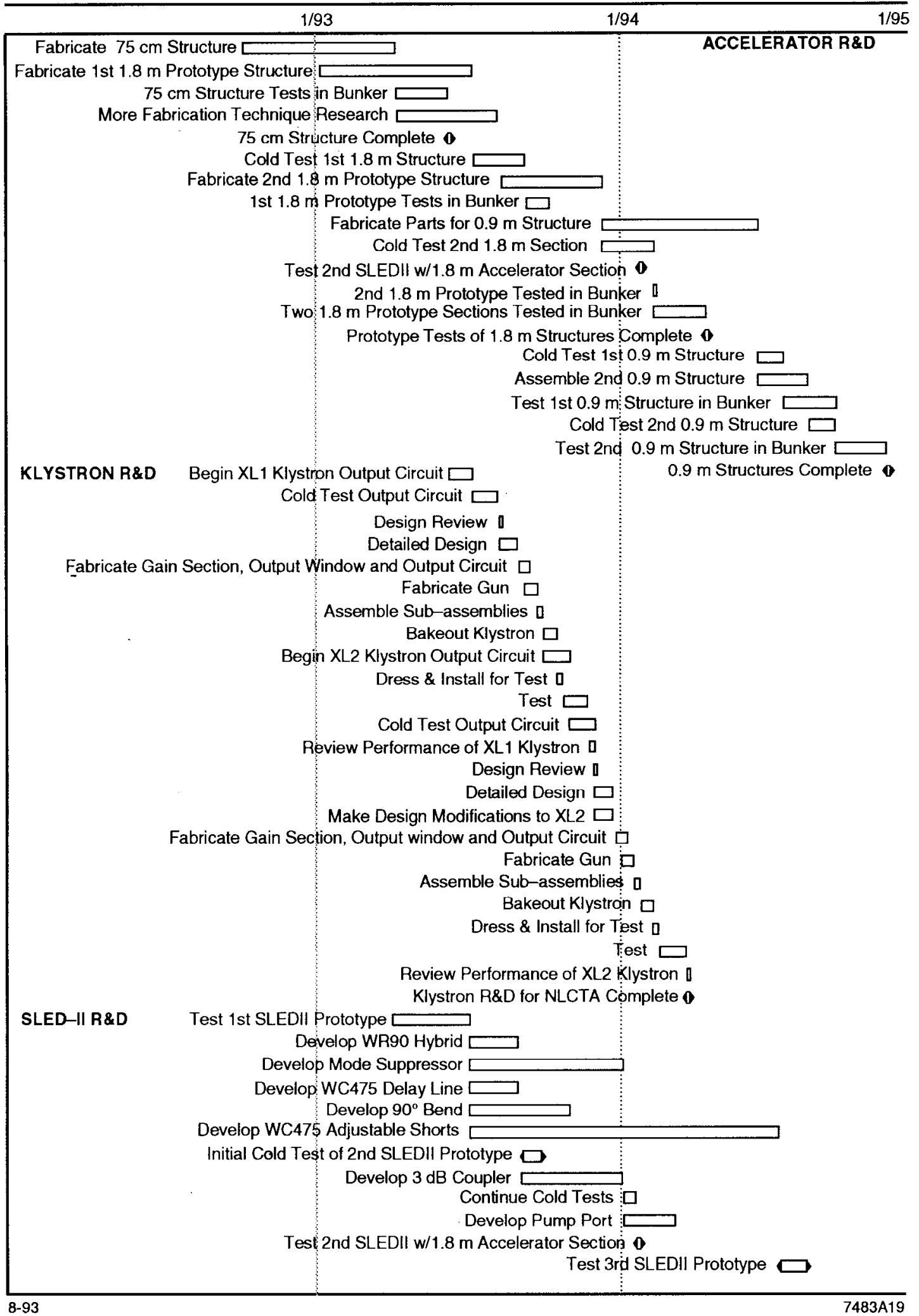

Figure 30. Project-related R\&D schedule. 


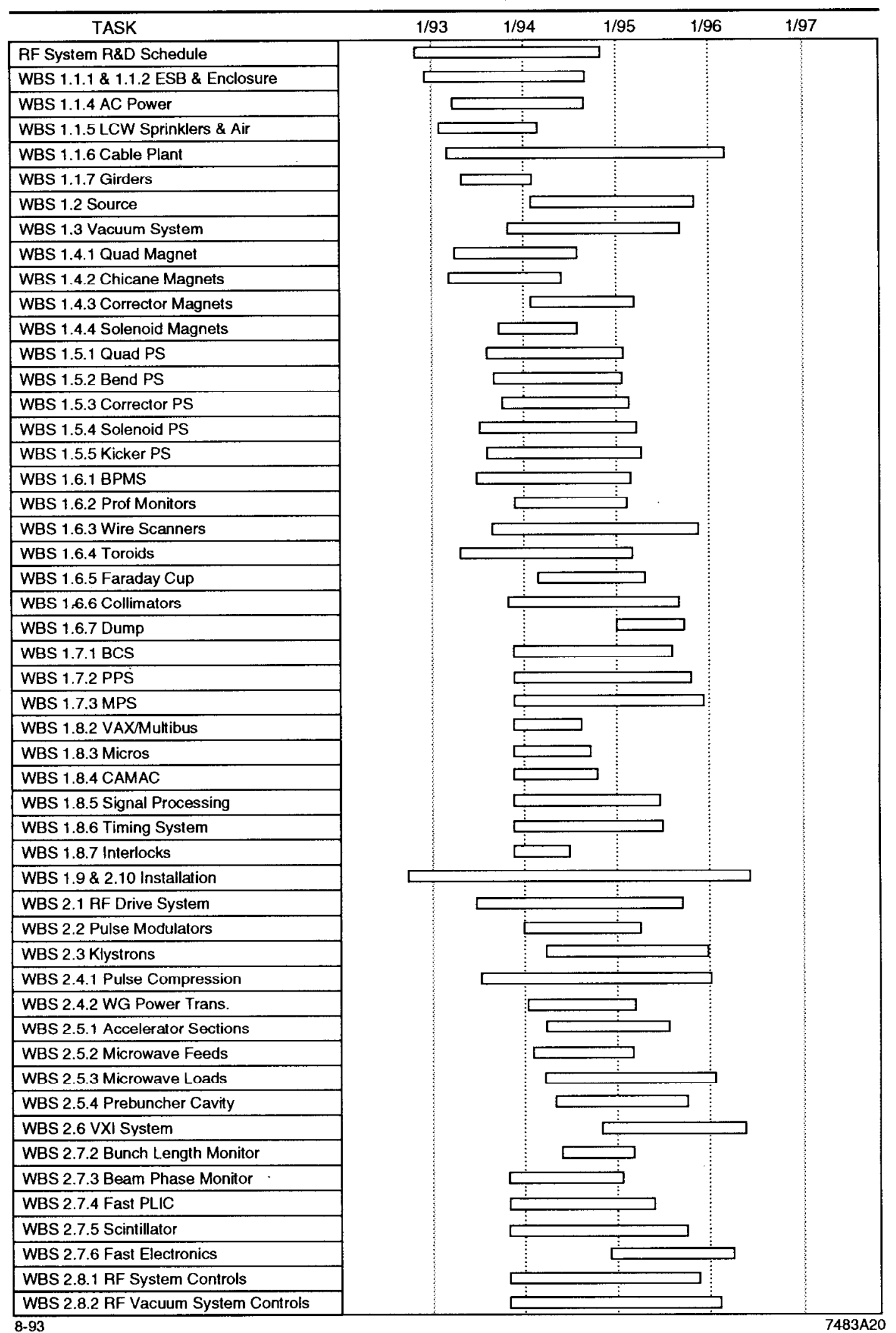

Figure 31. Project schedule. 


\subsection{Quality Assurance}

Quality assurance is the responsibility of all line managers on the NLCTA Project and will be implemented in accordance with the SLAC Institutional Quality Assurance Program Plan. ${ }^{47}$ The NLCTA has budgeted for a part time QA manager who will report directly to NLCTA management, develop a Quality Implementing Procedure (QIP) for the project and be responsible for project compliance with the QIP. The QIP will delineate the roles of project management and specify how Quality Assurance will be implemented and maintained. The QIP will, as a minimum, specify:

- Personnel training and qualifications

- Documentation and records

- Schedules

- Design review

- Procurement

- Inspection and testing.

As described in previous sections, the NLCTA Management Team will publish specifications, tolerances and procedures though a series of NLCTA Notes. Schedules -for each major subsystem will include design review, inspection and testing as milestones along their critical paths in their respective schedules. All fabrication will be done with released documents, and parts will be accompanied through each stage of fabrication by "travelers" that document that compliance has been met. The project will include resident and outside experts in the review process and publish minutes which document the discussions. Reviewers will provide technical input, encourage the use of high quality engineering practices, and ensure that environmental, safety, and health standards have bcen incorporated into each design. 


\section{Acknowledgements}

The conceptual design of the Next Linear Collider Test Accelerator, presented in this report, is the result of the contributions of many physicists, engineers, designers, and support staff, working under the direction of the NLCTA Project Management Team at SLAC. Secretarial support was provided by Tanya Boysen and Marge Bangali. The illustrations were prepared by Terry Anderson, Sylvia MacBride, and Jim Wahl. 


\section{References}

1. High Energy Physics: Research Directions for the Decade (Proceedings of the DPF 1990 Summer Study, Snowmass, Colorado, June 25-July 13, 1990), edited by E. L. Berger (World Scientific, River Edge, NJ, 1992).

2. High Energy Physics in the 1990's (Proceedings of the DPF 1988 Summer Study, Snowmass, Colorado, June 27-July 15, 1988), edited by Sharon Jensen (World Scientific, Teaneck, NJ, 1989).

3. Proceedings of The Second Workshop on Japan Linear Collider (JLC), Tsukuba, Japan, November 6-8, 1990, edited by S. Kawabata (KEK Proceedings 91-10, November 1991).

4. Proceedings of the Workshop on Physics and Experiments with Linear Colliders, Saariselka, Finland, September 9-14, 1991.

5. Burton Richter, "From the SLAC Linear Collider to the Next Linear Collider: A Status Report and Road Map" (SLAC-PUB-5736, February 1992); Ronald D. Ruth, "The Development of the Next Linear Collider at SLAC" (SLAC-PUB-5729, February 1992);

both papers to be published in Proceedings of the Workshop on Physics and Experiments with Linear Colliders, Saariselka, Finland, September 9-14, 1991.

6. Ronald D. Ruth, "The Next Linear Collider" (SLAC-PUB-5406, February 1991), published in Proceedings of the 18th Annual SLAC Summer Institute on Particle Physics: Gauge Bosons and Heavy Quarks, Stanford, California, 1990, edited by Jane F. Hawthorne (SLAC Report No. 378, Stanford, 1991), pp. 143-164; also published in Proceedings of the Joint US-CERN Particle Accelerator School on the Frontiers of Particle Beams: Intensity Limitations, Hilton Head, South Carolina, November 7-14, 1990, edited by M. Dienes, M. Month, and S. Turner (Springer-Verlag, New York, 1992).

7. Proceedings of the Third International Workshop on Linear Colliders, BINP, Protvino, USSR, September 17-27, 1991, edited by V. Balakin, S. Lepshokov, and N. Solyak (BINP, Protvino, 1990).

8. Proceedings of the 2nd International Workshop on Next-Generation Linear Collider, KEK, Tsukuba, Japan, March 28-April 5, 1990, edited by S. Kurokawa, H. Nakayama, and M. Yoshioka (KEK Internal Report 90-22, 1990).

9. Proceedings of the International Workshop on Next-Generation Linear Colliders, SLAC, Stanford, California, November 28-December 9, 1988 (SLAC Report No. 335, 1988).

10. R. D. Ruth, C. Adolphsen, K. L. Bane, R. F. Boyce, D. L. Burke, R. S. Callin, G. Caryotakis, R. Cassel, S. L. Clark, H. Deruyter, K. Fant, R. Fuller, S. Heifets, H. Hoag, R. Humphrey, S. Kheifets, R. Koontz, N. M. Kroll, T. Lavine, G. 
A. Loew, A. Menegat, R. H. Miller, C. Nantista, J. M. Paterson, C. Pearson, R. Phillips, J. Rifkin, J. Spencer, S. Tantawi, K. A. Thompson, A. Vlieks, V. Vylet, J. W. Wang, P. B. Wilson, A. Yeremian, and B. Youngman, "The Next Linear Collider Test Accelerator" (SLAC-PUB-6252), to be published in Proceedings of the 1993 IEEE Particle Accelerator Conference, op. cit.

11. J. M. Paterson, R. D. Ruth, C. Adolphsen, K. L. Bane, D. L. Burke, R. S. Callin, G. Caryotakis, R. L. Cassel, S. L. Clark, H. Deruyter, K. Fant, R. W. Fuller, S. A. Heifets, H. A. Hoag, R. Humphrey, A. J. Keicher, S. Kheifets, R. F. Koontz, N. M. Kroll, T. L. Lavine, G. A. Loew, A. Menegat, R. H. Miller, C. Nantista, M. E. Nordby, C. Pearson, J. Rifkin, J. Spencer, S. G. Tantawi, K. A. Thompson, A. E. Vlieks, V. Vylet, J. W. Wang, P. B. Wilson, A. D. Yeremian, and B. Youngman, "The Next Lincar Collider Test Accelerator" (SLAC-PUB5928), published in Proceedings of the 15th International Conference on HighEnergy Accelerators, Hamburg, Germany, July 20-24, 1992 [International Journal of Modern Physics A (Proceedings Supplement) 2B (1993)], pp. 815817.

12. C. Nantista, Z. D. Farkas, N. M. Kroll, T. L. Lavine, A. Menegat, R. D. Ruth, S. G. Tantawi, A. E. Vlieks, and P. B. Wilson, "High-Power SLED-II Pulse Compression with SLED-II at SLAC" (SLAC-PUB-6145), to be published in Proceedings of the 1993 IEEE Particle Accelerator Conference, Washington, DC, May 17-20, 1993.

13. P. B. Wilson, Z. D. Farkas, N. M. Kroll, T. L. Lavine, A. Menegat, C. Nantista, and R. D. Ruth, "Progress at SLAC on High-Power RF Pulse Compression" (SLAC-PUB-5866), published in Proceedings of the 15th International Conference on High Energy Particle Accelerators, op. cit., pp. 824-826.

14. N. M. Kroll, Z. D. Farkas, T. L. Lavine, A. Menegat, C. Nantista, R. D. Ruth, and P. B. Wilson, "A High Power SLED-II Pulse Compression System" (SLAC-PUB-5782), published in Proceedings of the Third European Particle Accelerator Conference, Berlin, Germany, March 24-28, 1992, edited by $\mathrm{H}$. Henke, H. Homeyer, and Ch. Petit-Jean-Genaz (Editions Frontières, Gif-surYvette, France, 1992), pp. 327-329.

15. P. B. Wilson, Z. D. Farkas, and R. D. Ruth, "SLED II: A New Method of RF Pulse Compression" (SLAC-PUB-5782), published in Proceedings of the 1990 Linear Accelerator Conference, Albuquerque, New Mexico, September 10-14, 1990 (Los Alamos National Laboratory Report LA-12004-C, 1991), pp. 204-206.

16. G. Caryotakis, R. Callin, K. Eppley, T. Lee, K. Fant, R. Fowkes, C. Pearson, R. Phillips, A. Vlieks, E. Wright, E. Lien, and G. Miram, "Development of Multimegawatt Klystrons for Linear Colliders" (SLAC-PUB-6168), to be published in Proceedings of the 1993 IEEE Particle Accelerator Conference, op. cit. 
17. G. Caryotakis, "Multimegawatt RF Power Sources for Linear Colliders" (SLACPUB-5508), published in Proceedings of the 1991 IEEE Particle Accelerator Conference, op. cit., pp. 2928-2932.

18. A. E. Vlieks, R. S. Callin, G. Caryotakis, K. S. Fant, W. R. Fowkes, T. G. Lee, and E. L. Wright, "100-MW Klystron Development at SLAC" (SLACPUB-5480), published in Proceedings of the 1991 IEEE Particle Accelerator Conference, op. cit., pp. 798-800.

19. H. Deruyter, Z. D. Farkas, H. Hoag, K. Ko, N. M. Kroll, G. A. Loew, R. H. Miller, R. B. Palmer, J. M. Paterson, K. Thompson, J. W. Wang, and P. B. Wilson, "Damped and Detuned Accelerator Structures" (SLAC-PUB-5322), published in Proceedings of the 1990 Linear Accelerator Conference, op. cit., pp. 132-134.

20. J. W. Wang, G. A. Loew, J. W. Simpson, E. Chojnacki, W. Gai, R. Konecny, and P. Schoessow, "Wakefield Measurements of SLAC Linac Structures at the Argonne AATF" (SLAC-PUB-5498), published in Proceedings of the 1991 IEEE Particle Accelerator Conference, op. cit., pp. 3219-3221.

21. R. H. Miller, C. Adolphsen, K. L. Bane, H. Deruyter, Z. D. Farkas, R. L. Gluckstern, H. Hoag, N. Holtkamp, K. Ko, N. M. Kroll, T. L. Lavine, G. A. Loew, E. M. Nelson, R. B. Palmer, J. M. Paterson, R. D. Ruth, K. Thompson, A. E. Vlieks, J. W. Wang, and P. B. Wilson, "Accelerator Structure Work for NLC" (SLAC-PUB-5865), published in Proceedings of the 15th International Conference on High Energy Accelerators, op. cit., pp. 833-835.

22. K. L. F. Bane and R. L. Gluckstern, "The Transverse Wakefield of a Detuned X-Band Accelerator Structure" (SLAC-PUB-5783), submitted to Particle Accelerators.

23. G. A. Loew and J. W. Wang, "RF Breakdown Studies in Room Temperature Electron Linac Structures" (SLAC-PUB-4647, May 1988), published in Proceedings of the 13th International Symposium on Discharges and Electrical Insulation in Vacuum, Paris, France, June 27-30, 1988, edited by J. M. Buzzi and A. Septier (Editions de Physique, Les Ulis, France, 1988).

24. J. W. Wang, R. A. Curry, H. Deruyter, H. A. Hoag, R. F. Koontz, G. A. Loew, A. Menegat, R. H. Miller, R. D. Ruth, A. E. Vlieks, C. Yoneda "High Gradient Studies on 11.4-GHz Copper Accelerator Structures" (SLAC-PUB-5900, August 1992), published in Proceedings of the 1992 Linear Accelerator Conference, Ottawa, Ontario, Canada, August 23-28, 1992 (Chalk River Laboratory Report AECL-10728, 1992), pp. 716-718.

25. K. Thompson and R. D. Ruth, "Controlling Transverse Multi-bunch Instabilities in Linacs of High Energy Linear Colliders," Physical Review D41, 964 (1990). 
26. T. Naito, M. Akemoto, H. Akiyama, H. Hayano, H. Matsumoto, J. Urakawa, and M. Yoshioka, "Generation of Multi-Bunch Beam with Thermionic Gun for the Japan Linear Collider" (KEK preprint 92-97), published in Proceedings of the 1992 Linear Accelerator Conference, op. cit., pp. 88-90.

27. P. G. O'Shea, S. C. Bender, B. E. Carlsten, J. W. Early, D. W. Feldman, R. B. Feldman, W. J. D. Johnson, A. H. Lumpkin, R. L. Sheffield, R. W. Springer, W. E. Stein, and L. M. Young, "Performance of the Photoinjector Accelerator for the Los Alamos Free Electron Laser," published in Proceedings of the 1991 IEEE Particle Accelerator Conference, op. cit., pp. 2754-2756.

28. K. Batchelor, H. Kirk, J. Sheehan, M. Woodle, and K. McDonald, "Development of a High Brightness Electron Gun for the Accelerator Test Facility at Brookhaven National Laboratory," published in Proceedings of the 1988 European Particle Accelerator Conference, Rome, Italy, June 7-11, 1988, edited by S. Tazzari (World Scientific, Singapore, 1989), pp. 954-957.

29. S. Heifets and S. Kheifets, "Optics of the NLCTA" (SLAC Advanced Accelerator Studies Note SLAC-AAS-70, April 1992).

30. S. A. Heifets and S. A. Kheifets, "Optics of a High-Gradient Linac" (SLAC Advanced Accelerator Studies Note SLAC-AAS-64, September 1991).

31. Theodore M. Jenkins, "Neutron and Photon Measurements Through Concrete from a 15-GeV Electron Beam on a Target: Comparison with Models and Calculations," Nuclear Instruments and Methods 159, 265 (1979).

32. J. C. Sheppard, J. E. Clendenin, R. H. Helm, M. J. Lee, R. H. Miller, and C. A. Blocker, "Emittance Calculations for the Stanford Linear Collider Injector," published in Proceedings of the 1983 IEEE Particle Accelerator Conference, Santa Fe, New Mexico (IEEE Transactions on Nuclear Science NS-30), pp. 2161-2163.

33. K. Harris, J. de Lamare, V. Nesterov, and R. Casscl, "600-kV Modulator Design for the SLAC Next Linear Collider Test Accelerator" (SLAC-PUB-5851), to be published in Proceedings of the 20th International Power Modulator Symposium, Myrtle Beach, South Carolina, June 23-25, 1992.

34. A. E. Vlieks, R. Callin, H. Deruyter, R. Early, K. S. Fant, Z. D. Farkas, W. R. Fowkes, C. Galloway, H. A. Hoag, G. A. Loew, T. L. Lavine, A. Menegat, R. H. Miller, D. Palmer, C. C. Pearson, R. D. Ruth, S. G. Tantawi, P. B. Wilson, J. W. Wang, C. Yoneda, N. Kroll, and C. Nantista, "Accelerator and RF System Development for NLC" (SLAC-PUB-6148), to be published in Proceedings of the 1993 IEEE Particle Accelerator Conference, op. cit.

35. T. L. Lavine, Z. D. Farkas, A. Menegat, R. H. Miller, C. Nantista, G. Spalek, and P. B. Wilson, "High-Power Radio-Frequency Binary Pulse-Compression Experiment at SLAC" (SLAC-PUB-5451), published in Proceedings of the 1991 IEEE Particle Accelerator Conference, San Francisco, California, May 6-9, 1991 (IEEE Catalog No. 91CH3038-7), pp. 652-654. 
36. H. A. Hoag, S. G. Tantawi, R. Callin, II. Deruyter, Z. D. Farkas, K. Ko, N. Kroll, T. L. Lavine, A. Menegat, and A. E. Vlieks, "Flower-Petal Mode Converter for NLC" (SLAC-PUB-6182), to be published in Proceedings of the 1993 IEEE Particle Accelerator Conference, op. cit.

37. A. P. King and E. A. Marcatili, "Transmission Loss Due to Resonance of Loosely-Coupled Modes in a Multi-Mode System," Bell System Technical Journal, Vol. 35, pp. 899-906, July 1956.

38. S. G. Tantawi, "Mode Selective Directional Coupler for NLC" (SLAC-PUB6174), to be published in Proceedings of the 1993 IEEE Particle Accelerator Conference, op. cit.

39. S. A. Heifets and S. A. Kheifets, "Longitudinal Electromagnetic Fields in an Aperiodic Structure" (SLAC-PUB-5907, September 1992), submitted to IEEE Transactions on Microwave Theory and Techniques; and "RF Pulse Transmission through an Accelerating Section" (SLAC Advanced Accelerator Studies Note SLAC-AAS-74, September 1992), to be published.

40. SLAC Radiological Control Manual, 1993, Article 131.

41. SLAC Radiological Control Manual, SLAC-I-720-0A05Z-001.

42. SLAC Environment Safety and Health Manual, SLAC-I-720-70100-100, Chapter 8 .

43. SLAC QACD Subcontractor Oversight Program, SLAC-I-770-0A17A-001-R001.

44. SLAC Emergency Preparedness Plan, SLAC-I-720-70000-105.

45. SLAC Emergency Preparedness Plan, 1991.

46. SLAC Hazard Communication Program, SLAC-I-720-0A06Z-001, 1992.

47. SLAC Institutional Quality Assurance Program Plan, SLAC-I-770-0A17M-001. 